\title{
Search for long-lived particles using displaced jets in proton-proton collisions at $\sqrt{s}=13 \mathrm{TeV}$
}

\author{
A. M. Sirunyan et al. \\ CMS Collaboration
}

(Received 2 December 2020; accepted 14 June 2021; published 30 July 2021)

\begin{abstract}
An inclusive search is presented for long-lived particles using displaced jets. The search uses a data sample collected with the CMS detector at the CERN LHC in 2017 and 2018, from proton-proton collisions at a center-of-mass energy of $13 \mathrm{TeV}$. The results of this search are combined with those of a previous search using a data sample collected with the CMS detector in 2016, yielding a total integrated luminosity of $132 \mathrm{fb}^{-1}$. The analysis searches for the distinctive topology of displaced tracks and displaced vertices associated with a dijet system. For a simplified model, where pair-produced long-lived neutral particles decay into quark-antiquark pairs, pair production cross sections larger than $0.07 \mathrm{fb}$ are excluded at 95\% confidence level (C.L.) for long-lived particle masses larger than $500 \mathrm{GeV}$ and mean proper decay lengths between 2 and $250 \mathrm{~mm}$. For a model where the standard model-like Higgs boson decays to two long-lived scalar particles that each decays to a quark-antiquark pair, branching fractions larger than $1 \%$ are excluded at $95 \%$ C.L. for mean proper decay lengths between $1 \mathrm{~mm}$ and $340 \mathrm{~mm}$. A group of supersymmetric models with pair-produced long-lived gluinos or top squarks decaying into various finalstate topologies containing displaced jets is also tested. Gluino masses up to $2500 \mathrm{GeV}$ and top squark masses up to $1600 \mathrm{GeV}$ are excluded at 95\% C.L. for mean proper decay lengths between 3 and $300 \mathrm{~mm}$. The highest lower bounds on mass reach $2600 \mathrm{GeV}$ for long-lived gluinos and $1800 \mathrm{GeV}$ for long-lived top squarks. These are the most stringent limits to date on these models.
\end{abstract}

DOI: 10.1103/PhysRevD.104.012015

\section{INTRODUCTION}

The existence of long-lived particles (LLPs) that have macroscopic decay lengths is a common feature in both the standard model (SM) and beyond-the-SM (BSM) scenarios. There are numerous alternative BSM physics cases for the production of LLPs at the CERN LHC. Examples include, but are not limited to: split supersymmetry (SUSY) [1-7], where the gluino decays are suppressed by heavy scalars; SUSY with weak $R$-parity violation (RPV) [8-12], where the decays of the lightest supersymmetric particle are suppressed by small RPV couplings; SUSY with gaugemediated SUSY breaking (GMSB) [13-15], where the decays of the next-to-lightest supersymmetric particle are suppressed by a large SUSY breaking scale; "stealth SUSY" [16,17]; "hidden valley" models [18-20]; dark matter models [21-28]; models with heavy neutral leptons that have small mixing parameters [29-32]; and models incorporating neutral naturalness [33-38]. In the examples

*Full author list given at the end of the article.

Published by the American Physical Society under the terms of the Creative Commons Attribution 4.0 International license. Further distribution of this work must maintain attribution to the author(s) and the published article's title, journal citation, and DOI. Funded by SCOAP ${ }^{3}$. listed above, it is very common for the LLPs to further decay into final states containing jets, giving rise to displaced-jets signatures.

Given the large variety of the BSM scenarios that lead to displaced-jets signatures, it is important to make the displaced-jets search as model independent as possible. In this paper, we present an inclusive search for LLPs decaying into jets, with at least one LLP having a decay vertex within the tracker acceptance, but which is displaced from the production vertex by up to $550 \mathrm{~mm}$ in the plane transverse to the beam direction. The search looks for a pair of jets known as dijets, where the jets are clustered from energy deposits in the calorimeters. For jets arising from the decay of an LLP, the associated tracks are usually displaced from the primary vertices (PVs), and the decay vertex can be reconstructed from the displaced tracks. The properties of the tracks and the decay vertex can provide discrimination power to distinguish long-lived signals from SM backgrounds. As mentioned above, a large number of models predict LLPs decaying into displaced jets. Our tests for some of these will be discussed in detail in Sec. III.

Events used in this analysis were collected with the CMS detector [39] at the LHC from proton-proton ( $p p)$ collisions at a center-of-mass energy of $13 \mathrm{TeV}$ in 2017 and 2018, corresponding to an integrated luminosity of $95.9 \mathrm{fb}^{-1}$. The results are combined with those of a 
previous displaced-jets search using the events collected in 2016 [40], yielding a total integrated luminosity of $132 \mathrm{fb}^{-1}$. For the models that were not studied in the 2016 displaced-jets search, additional simulated signal samples have been produced following the 2016 run condition of the CMS detector. These additional samples are then processed with the reconstruction and selection procedures described in Ref. [40] to compute the additional signal yields and systematic uncertainties for the 2016 data that are used in the combination.

Compared to the 2016 displaced-jets search, a set of new techniques that significantly improves the sensitivity to long-lived signatures is implemented in this analysis. The new techniques include one additional dedicated trigger aimed at selecting events containing displaced jets to recover efficiencies for high-mass LLPs, an auxiliary nuclear interactions (NIs) veto map to improve background rejection, a dedicated variable based on the sum of signed impact parameters of the tracks assigned to the displaced vertex, and the use of machine learning techniques to improve signal-to-background discrimination. With these new techniques, compared to the 2016 search, we have reduced the background rate by approximately a factor of three, while significantly increasing the signal efficiencies for almost all signal points in different LLP models. Results of searches for similar LLP signatures with hadronic decays at $\sqrt{s}=13 \mathrm{TeV}$ have also been reported by ATLAS [41-45] and CMS [46-48].

The paper is organized as follows. A brief description of the CMS detector is introduced in Sec. II. The data and the simulated samples are described in Sec. III. Section IV details the event reconstruction and the preselection criteria. Section V describes the event selections and the background estimation methods. The systematic uncertainties are summarized in Sec. VI. The observation and the interpretation of the results are described in Sec. VII. The paper is summarized in Sec. VIII.

\section{THE CMS DETECTOR}

The central feature of the CMS apparatus is a superconducting solenoid of $6 \mathrm{~m}$ internal diameter, providing a magnetic field of 3.8 T. Within the solenoid volume are a silicon pixel and strip tracker, a lead tungstate crystal electromagnetic calorimeter (ECAL), and a brass and scintillator hadron calorimeter (HCAL), each composed of a barrel and two endcap detectors. Muons are detected in gas-ionization chambers embedded in the steel flux-return yoke outside the solenoid.

The silicon tracker measures charged particles within the pseudorapidity range $|\eta|<2.5$. During the LHC run in 2017 and 2018, the silicon tracker consisted of 1856 silicon pixel and 15148 silicon strip detector modules, and it occupies a cylindrical volume around the interaction point with a length of $5.8 \mathrm{~m}$ and a diameter of $2.6 \mathrm{~m}$. For nonisolated particles with $1<p_{\mathrm{T}}<10 \mathrm{GeV}$ and $|\eta|<1.4$, the track resolutions are typically $1.5 \%$ in $p_{\mathrm{T}}$, and 25-75 $\mu \mathrm{m}$ in the transverse impact parameter [49].

In the region $|\eta|<1.74$, the HCAL cells have widths of $\Delta \eta=0.087$ in pseudorapidity and $\Delta \phi=0.087$ in azimuth. In the $\eta-\phi$ plane, and for $|\eta|<1.48$, the HCAL cells map on to $5 \times 5$ arrays of ECAL crystals to form calorimeter towers projecting radially outward from the nominal interaction point. For $|\eta|>1.74$, the coverage of the towers increases progressively to a maximum of 0.174 in $\Delta \eta$ and $\Delta \phi$. Within each tower, the energy deposits in ECAL and HCAL cells are summed to define the calorimeter tower energies, and are subsequently used to provide the energies and directions of hadronic jets.

Events of interest are selected using a two-tiered trigger system [50]. The first level, composed of custom hardware processors, uses information from the calorimeters and muon detectors to select events at a rate of around $100 \mathrm{kHz}$ within a time interval of less than $4 \mu \mathrm{s}$. The second level, known as the high-level trigger (HLT), consists of a farm of processors running a version of the full event reconstruction software optimized for fast processing, and reduces the event rate to around $1 \mathrm{kHz}$ before data storage.

A more detailed description of the CMS detector, together with a definition of the coordinate system used and the relevant kinematic variables, can be found in Ref. [39].

\section{DATASETS AND SIMULATED SAMPLES}

Data were collected with two dedicated triggers aimed at selecting events containing displaced jets. At the HLT, jets are reconstructed from the energy deposits in the calorimeter towers, clustered using the anti- $k_{\mathrm{T}}$ algorithm [51,52] with a distance parameter of 0.4. In this process, the contribution from each calorimeter tower is assigned a momentum, the magnitude and the direction of which are given by the energy measured in the tower and the coordinates of the tower. The raw jet energy is obtained from the sum of the tower energies, and the raw jet momentum from the vector sum of the tower momenta, which results in a nonzero jet mass. The raw jet energies are then corrected [53] to establish a uniform relative response of the calorimeter in $\eta$ and a calibrated absolute response in transverse momentum $p_{\mathrm{T}}$.

Identification of the $\mathrm{PV}$ is a prerequisite for the selection of displaced jets at the HLT. Events may contain multiple $\mathrm{PVs}$, corresponding to multiple $p p$ collisions occurring in the same bunch crossing. The candidate vertex with the largest value of summed physics-object $p_{\mathrm{T}}^{2}$ is taken to be the primary $p p$ interaction vertex. The physics objects are the jets, clustered using the jet finding algorithm [51,52] with the tracks assigned to candidate vertices as inputs, and the associated missing transverse momentum, taken as the negative vector sum of the $p_{\mathrm{T}}$ of those jets. More details are given in Sec. 9.4.1 of Ref. [54].

The first trigger, referred to as the "displaced" trigger, requires $H_{\mathrm{T}}>430 \mathrm{GeV}$, where $H_{\mathrm{T}}$ is the scalar sum of the 
jet $p_{\mathrm{T}}$ for all jets that have $p_{\mathrm{T}}>40 \mathrm{GeV}$ and $|\eta|<2.5$ in the event. The trigger also requires the presence of at least two jets, with the following requirements satisfied for each jet:

(i) $p_{\mathrm{T}}>40 \mathrm{GeV}$ and $|\eta|<2.0$;

(ii) at most two associated prompt tracks with $p_{\mathrm{T}}>1 \mathrm{GeV}$, where prompt tracks are those having a transverse impact parameter $\left(\mathrm{IP}_{2 \mathrm{D}}\right)$ with respect to the leading PV smaller than $1.0 \mathrm{~mm}$; and

(iii) at least one associated displaced track with $p_{\mathrm{T}}>1 \mathrm{GeV}$, where a displaced track is a track having an $\mathrm{IP}_{2 \mathrm{D}}$ larger than $0.5 \mathrm{~mm}$ and an impact parameter significance $\left(\operatorname{Sig}\left[\mathrm{IP}_{2 \mathrm{D}}\right]\right)$ larger than 5.0. The significance is defined as the ratio between the impact parameter and its uncertainty.

The second trigger, referred to as the "inclusive" trigger, requires $H_{\mathrm{T}}>650 \mathrm{GeV}$ and the presence of at least two jets, each of them satisfying:

(i) $p_{\mathrm{T}}>60 \mathrm{GeV}$ and $|\eta|<2.0$; and

(ii) at most two associated prompt tracks with $p_{\mathrm{T}}>1 \mathrm{GeV}$.

The displaced trigger is more efficient for low-mass LLPs, while the inclusive trigger is designed to recover the trigger efficiency for high-mass LLPs with small ( $\lesssim 3 \mathrm{~mm})$ or large $(\gtrsim 300 \mathrm{~mm})$ mean proper decay lengths $\left(c \tau_{0}\right)$.

The background sources in this search include NIs between outgoing particles and detector material, longlived SM hadrons, and misreconstructed displaced vertices formed by accidentally crossing tracks. The background events mainly arise from SM events containing jets produced through the strong interaction, referred to as quantum chromodynamics (QCD) multijet events. The QCD multijet Monte Carlo (MC) sample is simulated at leading order with MADGRAPH5_aMC@NLO 2.4.2 [55]. Parton showering and hadronization are simulated with PYTHIA8.226 [56]. The matching of jets from the matrix element calculations and parton shower jets is achieved using the MLM algorithm [57]. The PYTHIA parameters for the underlying event modeling are set to be the CP5 tune [58]. The set of parton distribution functions (PDFs) used for the production is the NNPDF3.1 NNLO PDF set [59]. The QCD multijet MC sample is mainly used to inspire the analysis strategy and to estimate systematic uncertainties, while the background estimation for this search is purely determined from data.

Feynman diagrams for the benchmark models studied in this paper are summarized in Fig. 1. One of the benchmark signal models is a simplified model, where long-lived scalar neutral particles $X$ are pair produced through a scattering process mediated by an off-shell $Z$ boson. In this model, each $\mathrm{X}$ particle decays to a quark-antiquark pair, assuming equal branching fractions to $u, d, s, c$, and $b$ quark pairs. The decays to top quark pairs are excluded to provide a simple final-state topology for this model, but it was important that the analysis strategy would still be sensitive to a variety of other models. We checked the impact on the signal efficiencies of excluding decays to top quark pairs, and found it to be small, where the relative changes of the signal efficiencies are generally at the order of a few percents. This model is referred to as the jet-jet model. The samples are produced with different resonance masses ranging from 50 to $1500 \mathrm{GeV}$, and with different proper decay lengths ranging from 1 to $10^{4} \mathrm{~mm}$.

Another signature we consider is the case where LLPs arise from exotic decays of an SM-like Higgs boson, which can happen in many BSM scenarios (a review can be found in the Sec. IV. 6. 6 of Ref. [60]), including "hidden valley" models [18,19], twin Higgs models [36], and the folded SUSY model [61]. For the simulation, we use POWHEG 2.0 [62-65] to generate events containing a $125 \mathrm{GeV}$ Higgs boson produced through gluon-gluon fusion. The $125 \mathrm{GeV}$ Higgs boson then decays to two long-lived scalar particles $\mathrm{S}$, and each scalar particle then decays to a quark-antiquark pair. Two scenarios are considered; in the first scenario the scalar particle has a branching fraction of $100 \%$ to decay to a down quark-antiquark pair, while in the second one the scalar particle has a branching fraction of $100 \%$ to decay to a bottom quark-antiquark pair. The samples are produced with the scalar particle mass $m_{\mathrm{S}}$ set to be 15,40 , or $55 \mathrm{GeV}$, while the $c \tau_{0}$ of $\mathrm{S}$ varies from 1 to $3000 \mathrm{~mm}$.

We also consider a group of SUSY models with different final-state topologies. The first one is a GMSB SUSY model [66] in the general gauge mediation scenario [14,15], where gluinos are pair produced and the gravitino is the lightest SUSY particle, while the gluino is the next-tolightest supersymmetric particle. After the gluino is produced, it decays to a gluon and a gravitino, producing a single displaced jet and missing transverse momentum. This decay is suppressed by the SUSY-breaking scale, and therefore the gluino is long lived. The model in which this process occurs is referred to as the $\tilde{g} \rightarrow g \tilde{G}$ model. The samples are produced with gluino masses from 800 to $2500 \mathrm{GeV}$, and with the $c \tau_{0}$ of the gluino varying from 1 to $10^{4} \mathrm{~mm}$.

The second SUSY model we consider is a mini-split SUSY model [6,7], referred to as the $\tilde{g} \rightarrow q \bar{q} \tilde{\chi}_{1}^{0}$ model. In this model the gluino decays to a quark-antiquark pair and the lightest neutralino $\left(\tilde{\chi}_{1}^{0}\right)$, with equal branching fractions to $u, d, s$, and $c$ quark pairs. This decay is mediated by a squark, which is much heavier than the gluino. The squark's large mass suppresses the gluino decay, making it long lived. The mass of the neutralino is assumed to be $100 \mathrm{GeV}$, the samples are produced with gluino masses from 1400 to $3000 \mathrm{GeV}$, and the $c \tau_{0}$ of the gluino varies from 1 to $10^{4} \mathrm{~mm}$.

The third SUSY model is an RPV SUSY model [67] with minimal flavor violation, where gluinos are pair produced and long lived. Each long-lived gluino decays to top, bottom, and strange antiquarks through the RPV coupling $\lambda_{323}^{\prime \prime}$ and the mediation of a virtual top squark [12], 

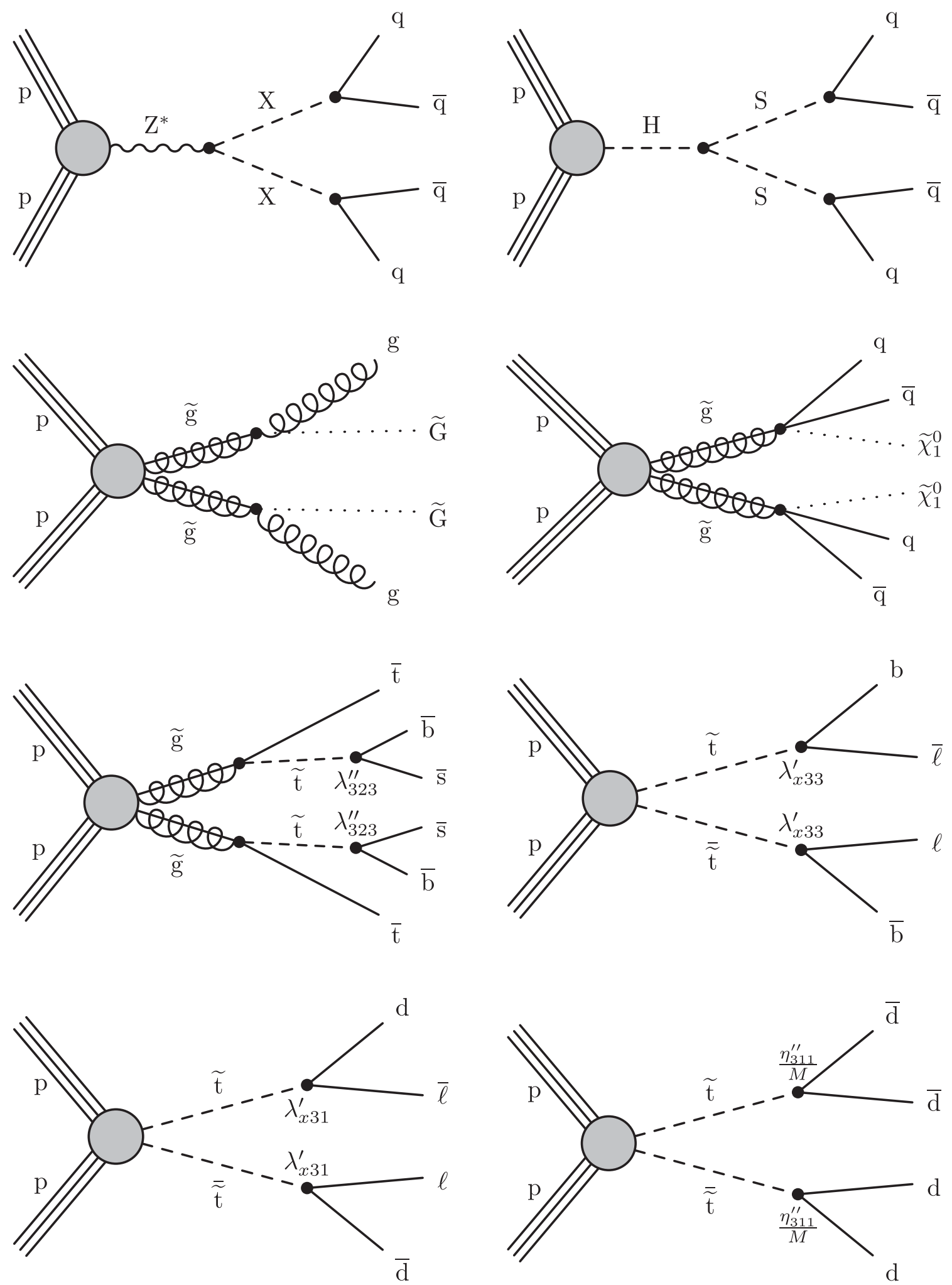

FIG. 1. The Feynman diagrams for the different long-lived models considered, including the jet-jet model (upper left), models with an exotic decay of the SM-like Higgs boson (upper right), general gauge mediation models with $\tilde{g} \rightarrow g \tilde{G}$ decay (second row, left), mini-split SUSY with $\tilde{g} \rightarrow q \bar{q} \tilde{\chi}_{1}^{0}$ decay (second row, right), RPV SUSY with $\tilde{g} \rightarrow t b s$ decay (third row, left), RPV SUSY with $\tilde{t} \rightarrow b \ell$ decay (third row, right), RPV SUSY with $\tilde{t} \rightarrow d \ell$ decay (lower left), and dRPV SUSY with $\tilde{t} \rightarrow \bar{d} \bar{d}$ decay (lower right). 
leading to a multijet final-state topology. This model is referred to as the $\tilde{g} \rightarrow t b s$ model. The samples are produced with gluino masses from 1200 to $3000 \mathrm{GeV}$, and a $c \tau_{0}$ varying from 1 to $10^{4} \mathrm{~mm}$.

We also consider two other RPV SUSY models [68] with semileptonic decays, in which long-lived top squarks are pair produced, and each top squark decays to a bottom quark (down quark) and a charged lepton via RPV couplings $\lambda_{133}^{\prime}, \lambda_{233}^{\prime}$, and $\lambda_{333}^{\prime}\left(\lambda_{131}^{\prime}, \lambda_{231}^{\prime}\right.$, and $\left.\lambda_{331}^{\prime}\right)$ [12]. The decay rate to each of the three lepton flavors is assumed to be equal. The two models are referred to as the $\tilde{t} \rightarrow b \ell$ $(\tilde{t} \rightarrow d \ell)$ models. The samples are produced with different top squark masses from 600 to $2000 \mathrm{GeV}$, and a $c \tau_{0}$ varying from 1 to $10^{4} \mathrm{~mm}$.

Finally, we consider another SUSY model, referred to as the $\tilde{t} \rightarrow \bar{d} \bar{d}$ model, motivated by dynamical RPV (dRPV) $[69,70]$, where long-lived top squarks are pair produced, and each top squark decays to two down antiquarks via a nonholomorphic RPV coupling $\eta_{311}^{\prime \prime}$ [71]. The nonholomorphic RPV coupling is suppressed by some large scale $M$, thus giving rise to long lifetimes. The samples are produced with different top squark masses from 800 to $1800 \mathrm{GeV}$, and a $c \tau_{0}$ varying from 1 to $10^{4} \mathrm{~mm}$.

PYTHIA8.226 is used for the production of the signal samples, and the PDF set used for the production is NNPDF3.1 LO. For SUSY-particle production, the PYTHIA8.226 simulation is cross checked with MADGRAPH5_aMC@NLO 2.4.2 for representative signal points, where the MADGRAPH5_aMC@NLO simulation is performed at LO with up to two additional outgoing partons. The resulting signal efficiencies are found to be consistent within the statistical uncertainties. The PYTHIA parameters for the underlying event modeling are set to be the CP2 tune [58]. In the SUSY models, a longlived gluino or top squark can form a hadronic state through strong interactions, an $R$-hadron $[9,72,73]$, which is simulated with PYTHIA. The interactions of the $R$-hadron with matter were studied following the simulation described in Ref. [74,75], and were found to have negligible impact on this analysis, since they have very little influence on the vertex reconstruction.

The simulated background and signal events are processed with a GEANT4-based [76] simulation for the detailed CMS detector response. To take into account the effects of additional $p p$ interactions within the same or nearby bunch crossings ("pileup"), additional minimum-bias events are overlaid on the simulated events to match the pileup distribution observed in the data.

\section{EVENT RECONSTRUCTION AND PRESELECTION}

This search examines dijet candidates in a given event. The algorithms for the offline jet reconstruction and PV selection are the same as those applied at the HLT (as described in Sec. III), except that the full offline information is used. To make sure that the online $H_{\mathrm{T}}$ and jet $p_{\mathrm{T}}$ requirements in the displaced-jet triggers reach full efficiency, we apply selections on the offline $H_{\mathrm{T}}$ of the event as well as on the $p_{\mathrm{T}}$ and $\eta$ of each jet. After the trigger selection, if an event passes the displaced trigger, we require the event to have offline $H_{\mathrm{T}}>500 \mathrm{GeV}$, and dijet candidates are formed from all possible pairs of jets in the event, with the jets satisfying $p_{\mathrm{T}}>50 \mathrm{GeV}$ and $|\eta|<2.0$. On the other hand, if an event only passes the inclusive trigger, it is required to have offline $H_{\mathrm{T}}>700 \mathrm{GeV}$, and the dijet candidates are formed from all possible pairs of jets in the event, with the jets satisfying $p_{\mathrm{T}}>80 \mathrm{GeV}$ and $|\eta|<2.0$.

In this search, the track candidates are required to have $p_{\mathrm{T}}>1 \mathrm{GeV}$ and to be high-purity tracks. The high-purity selection is based on track information (such as the normalized $\chi^{2}$ of the track fit, the impact parameters, and the number of hits in different tracker layers) to reduce the fraction of misreconstructed tracks, and the selection is optimized separately for each iteration of the tracking [49], so that it is efficient for selecting tracks with different displacements. More details of the high-purity selection can be found in Ref. [49]. The $\eta$ and $\phi$ of a given track are determined by the direction of its momentum vector at the closest approach point to the leading PV. For a given dijet candidate, we associate track candidates with each jet by requiring that $\Delta R<0.5$, where $\Delta R=\sqrt{(\Delta \eta)^{2}+(\Delta \phi)^{2}}$ is the angular distance between the jet axis and the track direction. When a track satisfies $\Delta R<0.5$ for both jets, it is associated with the jet giving the smaller $\Delta R$.

After associating track candidates with each jet, the next step is to reconstruct a secondary vertex (SV) for each dijet candidate. From all the tracks associated with a dijet candidate, we select displaced tracks that satisfy $\mathrm{IP}_{2 \mathrm{D}}>$ $0.5 \mathrm{~mm}$ and $\operatorname{Sig}\left[\mathrm{IP}_{2 \mathrm{D}}\right]>5.0$. We then attempt to reconstruct an SV from these displaced tracks using an adaptive vertex fitter algorithm [77]. The reconstructed SV is not required to have associated tracks from both jets, so that the search can be sensitive to the models where the LLP decays to a single displaced jet. To improve the signal-tobackground discrimination, we implement a set of preselection criteria on the dijet/SV candidates, which are described in the rest of this section.

To ensure the quality of the vertex reconstruction, the SV is selected only if it is reconstructed with a $\chi^{2}$ per degree of freedom $\left(\chi^{2} / \mathrm{n}_{\mathrm{dof}}\right)$ of less than 5.0. In order to suppress long-lived SM mesons and baryons, the invariant mass of the vertex is required to be larger than $4 \mathrm{GeV}$, and the transverse momentum of the vertex is required to be larger than $8 \mathrm{GeV}$, where the four-momentum of the vertex is calculated assuming the charged pion mass for all assigned tracks.

We only consider dijet candidates that have a reconstructed SV satisfying the above requirements. Furthermore, SVs in background events tend to have only one track with a high value for $\mathrm{IP}_{2 \mathrm{D}}$, corresponding to the tail of the impact parameter distribution. We therefore consider the track with 
the second-highest $\operatorname{Sig}\left[\mathrm{IP}_{2 \mathrm{D}}\right]$ among the tracks that are assigned to the SV, since this provides a more sensitive discriminant for identifying displaced jets. We require the second-highest $\operatorname{Sig}\left[\mathrm{IP}_{2 \mathrm{D}}\right]$ to be larger than 15 .

We also compute another quantity $\epsilon$, which is the ratio between the sum of energy for all the tracks assigned to the SV and the sum of the energy for all the tracks associated with the two jets:

$$
\epsilon=\frac{\sum_{\text {track } \in \mathrm{SV}} E_{\text {track }}}{\sum_{\text {track } \in \text { dijet }} E_{\text {track }}} .
$$

Since $\epsilon$ is expected to be large for displaced-jet signatures, dijet candidates with $\epsilon$ smaller than 0.15 are rejected.

An additional variable, $\zeta$, is defined to characterize the contribution of prompt activity to the jets. For each track associated with a jet, we identify the PV (including the leading PV and the pileup vertices) with the minimum three-dimensional (3D) impact parameter significance to the track. If this minimum value is smaller than 5, we assign the track to this PV. Then for each jet, we compute the track energy contribution from each PV, and the PV with the largest track energy contribution to the jet is chosen. Finally, we define $\zeta$ as

$$
\zeta=\frac{\sum_{\text {track } \in \mathrm{PV}_{1}} E_{\text {track }_{1}}^{\mathrm{Jet}_{1}}+\sum_{\text {track } \in \mathrm{PV}_{2}} E_{\text {track }}^{\mathrm{Jet}_{2}}}{E_{\mathrm{Jet}_{1}}+E_{\mathrm{Jet}_{2}}},
$$

where $\sum_{\text {track } \in \mathrm{PV}_{i}} E_{\text {track }}^{\mathrm{Jet}_{i}}$ is the sum of the track energy coming from the most compatible PV for a given jet, while $E_{\mathrm{Jet}_{i}}$ is the energy of a given jet, thus $\zeta$ is the charged energy fraction of the dijet associated with the most compatible PVs. For displaced-jet signatures, $\zeta$ tends to be small since the jets are not compatible with PVs. Dijet candidates with $\zeta$ larger than 0.2 are rejected.

To suppress the background events arising from NIs in the tracker material, we compare the positions of the SVs with a map of the distribution of material in the inner tracker. The map was obtained from the distribution of NI candidate vertices, which are reconstructed using the adaptive vertex fitter on a sample of events collected with isolated single-muon triggers. The NI candidates are required to satisfy the following criteria:

(i) The tracks are required to be associated with dijet candidates, which are formed from the jets having $p_{\mathrm{T}}>10 \mathrm{GeV}$ and $|\eta|<2.0$;

(ii) The associated tracks must have $p_{\mathrm{T}}>0.2 \mathrm{GeV}$, high purity, $\mathrm{IP}_{2 \mathrm{D}}>0.5 \mathrm{~mm}$, and $\operatorname{Sig}\left[\mathrm{IP}_{2 \mathrm{D}}\right]>5.0$;

(iii) vertex track multiplicity is larger than 3 ;

(iv) the ratio $\epsilon$ of the energy sum for SV tracks to that for all tracks is less than 0.15 for the dijet candidate;

(v) vertex $L_{x y}$ significance is larger than 200 , where the transverse decay length $L_{x y}$ is the distance between the SV and the leading PV; and

(vi) vertex $\chi^{2} / \mathrm{n}_{\text {dof }}$ is smaller than 3.0.
After these selections, the distribution of the NI vertex candidates is transferred to an NI-veto map in the transverse plane, with $|x|$ and $|y|<25 \mathrm{~cm}$, as shown in Fig. 2. To suppress misreconstructed vertices and the displaced vertices produced by decaying long-lived SM mesons and baryons, we only select the region where the NI vertex density is above a threshold that varies for different layers of the pixel detector. In the NI-veto map, we can clearly see the structures of the beam pipe (at $r=\sqrt{x^{2}+y^{2}} \approx 23 \mathrm{~mm}$ ),
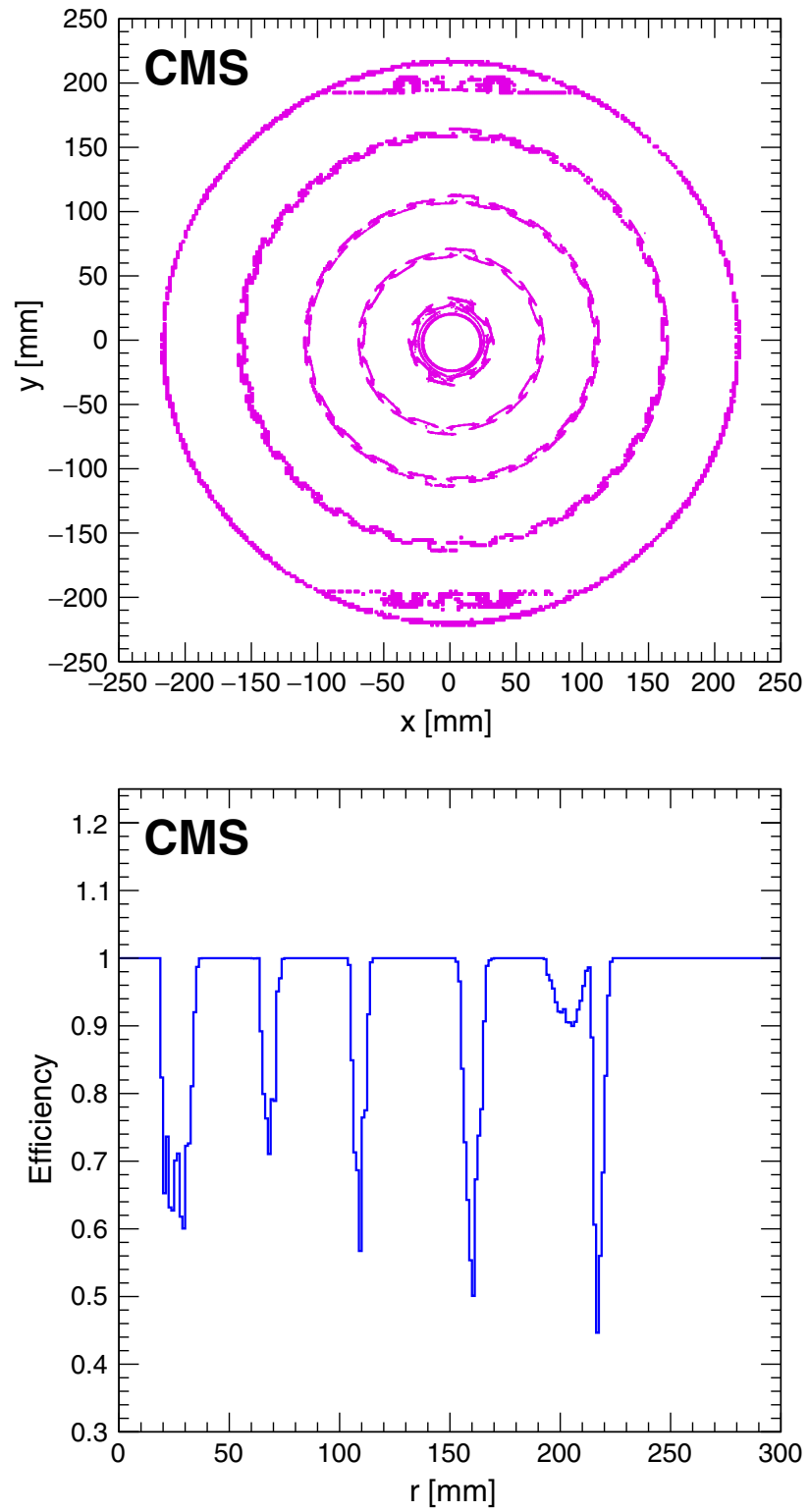

FIG. 2. Upper: the NI-veto map based on the NI vertex reconstruction in the 2017 and 2018 data collected by the CMS detector, the map corresponds to the geometry of the CMS pixel detector used in 2017-2018 data taking [79]. The structures of the different pixel layers can be clearly seen. Lower: the efficiency for a given vertex candidate to pass the NI-veto as a function of radius $r$. 
TABLE I. Summary of the preselection criteria.

\begin{tabular}{lc}
\hline \hline SV/dijet variable & Requirement \\
\hline Vertex $\chi^{2} / \mathrm{n}_{\text {dof }}$ & $<5.0$ \\
Vertex invariant mass & $>4 \mathrm{GeV}$ \\
Vertex transverse momentum & $>8 \mathrm{GeV}$ \\
Second largest IP $2 \mathrm{D}$ significance & $>15$ \\
$\epsilon$ (SV track energy fraction in the dijet) & $>0.15$ \\
$\zeta$ (energy fraction from compatible PVs) & $<0.20$ \\
Vertex position in the $x$ - $y$ plane & No overlap with \\
& the NI-veto map \\
\hline \hline
\end{tabular}

the four pixel layers (at $r \approx 29, \approx 68, \approx 109$, and $\approx 160 \mathrm{~mm}$ ), and the support rails (at $r \approx 200 \mathrm{~mm}$ ). In our search, any $\mathrm{SV}$ candidate that overlaps with the NI-veto map is rejected. The loss of the fiducial volume within $r<$ $300 \mathrm{~mm}$ due to the veto is around $4 \%$, and the efficiencies for signal events to pass this selection are generally well above $90 \%$. In the veto no requirement is placed on the $z$ coordinates of the SVs, but the impact of restricting the veto to the barrel region of the pixel detector $(|z|<27 \mathrm{~cm})$ is negligible on the signal efficiencies. A similar study on the structure of the CMS inner tracking system using a more sophisticated NI reconstruction technique with 2016 data has been reported in Ref. [78].

The preselection criteria for this search, summarized in Table I, are efficient for a wide range of long-lived models with different final-state topologies.

\section{EVENT SELECTION AND BACKGROUND PREDICTION}

After reconstructing the SV using the adaptive vertex fitter, we employ an auxiliary algorithm to check the consistency between the SV system and the dijet system. For each displaced track (having $\mathrm{IP}_{2 \mathrm{D}}>0.5 \mathrm{~mm}$, $\operatorname{Sig}\left[\mathrm{IP}_{2 \mathrm{D}}\right]>5.0$ ) associated with the dijet, we determine an expected decay point consistent with the displaced dijet hypothesis by finding the crossing point of the track helix and the dijet direction in the transverse $(x-y)$ plane. The dijet direction is the space direction of the four-momentumsum of the two jets, for which the production vertex of the two jets is taken to be the SV. For each crossing point, an expected transverse decay length $\left(L_{x y}^{\exp }\right)$ is computed with respect to the leading PV. The $L_{x y}^{\exp }$ is positive if the crossing point is at the same side of the dijet direction, otherwise it is negative. The associated displaced tracks are then clustered based on their $L_{x y}^{\exp }$, using a hierarchical clustering algorithm [80]. During the clustering, two clusters are merged when the smallest $L_{x y}^{\exp }$ difference between the two clusters is smaller than $15 \%$ of the $L_{x y}$ of the SV. After the clustering procedure is finished, if more than one cluster is formed, the one closest to the SV is selected. The cluster root-mean-square (RMS), taken to be the relative RMS of individual tracks $L_{x y}^{\exp }$ with respect to the SV $L_{x y}$ is computed to provide signal-to-background discrimination:

$$
\mathrm{RMS}_{\text {cluster }}=\sqrt{\frac{1}{N_{\text {tracks }}} \sum_{i=1}^{N_{\text {tracks }}} \frac{\left(L_{x y}^{\exp }(i)-L_{x y}\right)^{2}}{L_{x y}^{2}}},
$$

where $N_{\text {tracks }}$ is the number of tracks in the selected cluster.

For each track assigned to the $\mathrm{SV}$, a sign is given to the $\mathrm{IP}_{2 \mathrm{D}}$ and $\operatorname{Sig}\left[\mathrm{IP}_{2 \mathrm{D}}\right]$ based on the angle between the dijet direction and the impact parameter vector that points from the leading PV to the closest approach point (with respect to the leading PV) of the track in the transverse plane. The sign is positive if this angle is smaller than $\pi / 2$; otherwise the sign is negative. A new variable, $\kappa$, is then introduced as the signed $\operatorname{Sig}\left[\mathrm{IP}_{2 \mathrm{D}}\right]$ sum of the six leading tracks from the $\mathrm{SV}$ (where the tracks are ordered by the absolute values of their $\left.\operatorname{Sig}\left[\mathrm{IP}_{2 \mathrm{D}}\right]\right)$ :

$$
\kappa=\sum_{i=1}^{6} \operatorname{Sig}\left[\operatorname{IP}_{2 \mathrm{D}}\left(\operatorname{track}_{i}\right)\right] .
$$

If the track multiplicity is smaller than six, the sum is taken over all the tracks from the SV. For background processes, since the tracks assigned to the $\mathrm{SV}$ are uncorrelated with the dijet direction, the signed $\mathrm{Sig}\left[\mathrm{IP}_{2 \mathrm{D}}\right]$ of different tracks tend to cancel each other, therefore $\kappa$ peaks sharply around zero. On the other hand, for displaced jets that originate from the $\mathrm{SV}$, the directions of the tracks will be highly correlated with the dijet direction, therefore $\kappa$ is significantly different from zero and $|\kappa|$ tends to be large.

To improve signal-to-background discrimination and to define a region with signal events enriched, we proceed to construct a multivariate discriminant based on the following variables for the vertex/dijet candidates:

(i) Vertex track multiplicity;

(ii) Vertex $L_{x y}$ significance;

(iii) Cluster RMS;

(iv) The magnitude of the signed $\operatorname{Sig}\left[\mathrm{IP}_{2 \mathrm{D}}\right]$ sum of the six leading tracks $|\kappa|$.

The distributions of the four variables are shown in Fig. 3, with displaced-jet triggers, offline $H_{\mathrm{T}}$, and offline jet kinematic variables selections (described in Section IV) applied. For the multivariate discriminant we utilize the gradient boosted decision tree (GBDT) algorithm [81-83], with cross entropy as the loss function. The GBDT algorithm is implemented using the TMVA (toolkit for multivariate data analysis) package [84] interfaced with Scikit-learn [85]. Given the large cross section of the QCD multijet process and the relatively low $H_{\mathrm{T}}$ threshold of our displaced-jet triggers, the event count of the simulated QCD multijet sample (after preselections) is insufficient for the GBDT training, since it is much smaller than the number of expected QCD multijet events in the analyzed 

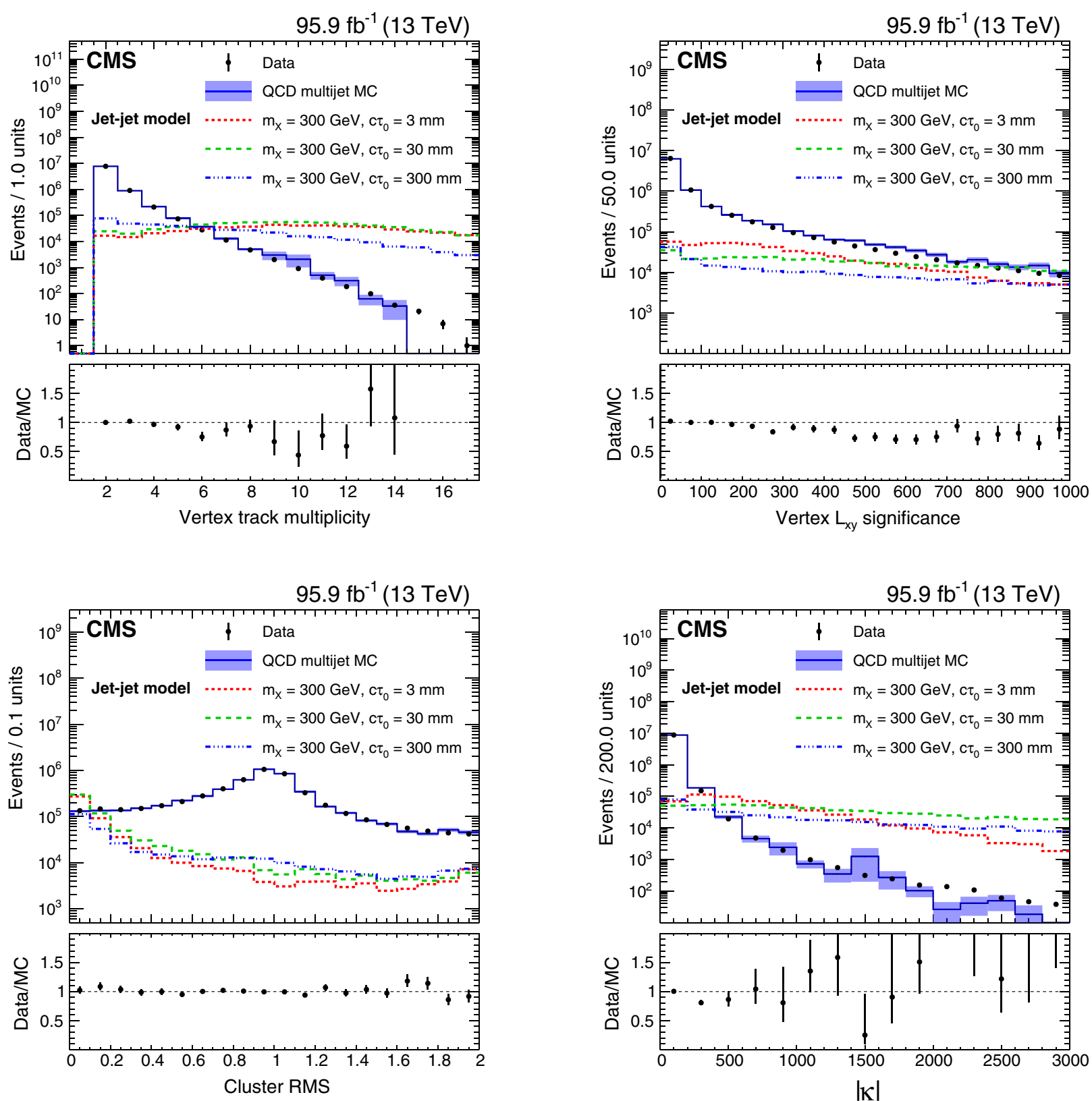

FIG. 3. The distributions of the vertex track multiplicity (upper left), vertex $L_{x y}$ significance (upper right), cluster RMS (lower left), and the magnitude of the signed $\operatorname{Sig}\left[\mathrm{IP}_{2 \mathrm{D}}\right]$ sum $|\kappa|$ (lower right), for data, simulated QCD multijet events, and simulated signal events. Data and simulated events are selected with the displaced-jet triggers and with the offline $H_{\mathrm{T}}$, jets $p_{\mathrm{T}}$, and $\eta$ selections applied. For a given event, if there is more than one SV candidate being reconstructed, the one with the largest vertex track multiplicity is chosen. If the track multiplicities are the same, the one with the smallest $\chi^{2} / \mathrm{n}_{\mathrm{dof}}$ is chosen. The lower panels show the ratios between the data and the simulated QCD multijet events. The blue shaded error bands and vertical bars represent the statistical uncertainties. Three benchmark signal distributions are shown (dashed lines) for the jet-jet model with $m_{\mathrm{X}}=300 \mathrm{GeV}$ and varying $c \tau_{0}$. For visualization purposes, each signal process is given a cross section that yields $10^{6}$ events produced in the analyzed data sample.

data sample. Therefore, for the background sample in the GBDT training, we use the data in the following region:

(i) events are selected by the displaced-jet triggers, and pass the offline $H_{\mathrm{T}}$ and jet kinematic variables selections;

(ii) $\epsilon<0.12$ for the dijet candidate, making this region orthogonal to the signal region; (iii) the veto using the NI-veto map is not applied;

(iv) all the other preselection criteria are satisfied.

For the signal sample in the GBDT training, simulated jetjet model events that pass the preselection criteria are used, with $m_{\mathrm{X}}=100,300$, and $1000 \mathrm{GeV}$, and with $c \tau_{0}=1,10$, $100,1000 \mathrm{~mm}$. If there is more than one dijet/SV candidate passing the selection criteria in a given event, the one with 
the largest track multiplicity is chosen for the training. If the track multiplicities are the same, the one with the smallest SV $\chi^{2} / \mathrm{n}_{\text {dof }}$ is chosen. An event weight is assigned separately to each signal point with a given $m_{\mathrm{X}}$ and $c \tau_{0}$ such that the sum of weights is identical for each point, thus each signal point has the same priority in the training. Twenty percent of the events in the signal and background samples are randomly selected to validate the performance of the GBDT and to make sure it is not overtrained.

The GBDT output values or scores for data, simulated QCD multijet events, and simulated signal events are shown in Fig. 4. The signal efficiencies for this search are measured with simulated signal events produced separately. The background prediction is purely based on some other control samples in data, which are different from the one used for the GBDT training. Although only the jet-jet model is used as the signal sample in the GBDT training, the GBDT is highly efficient in selecting

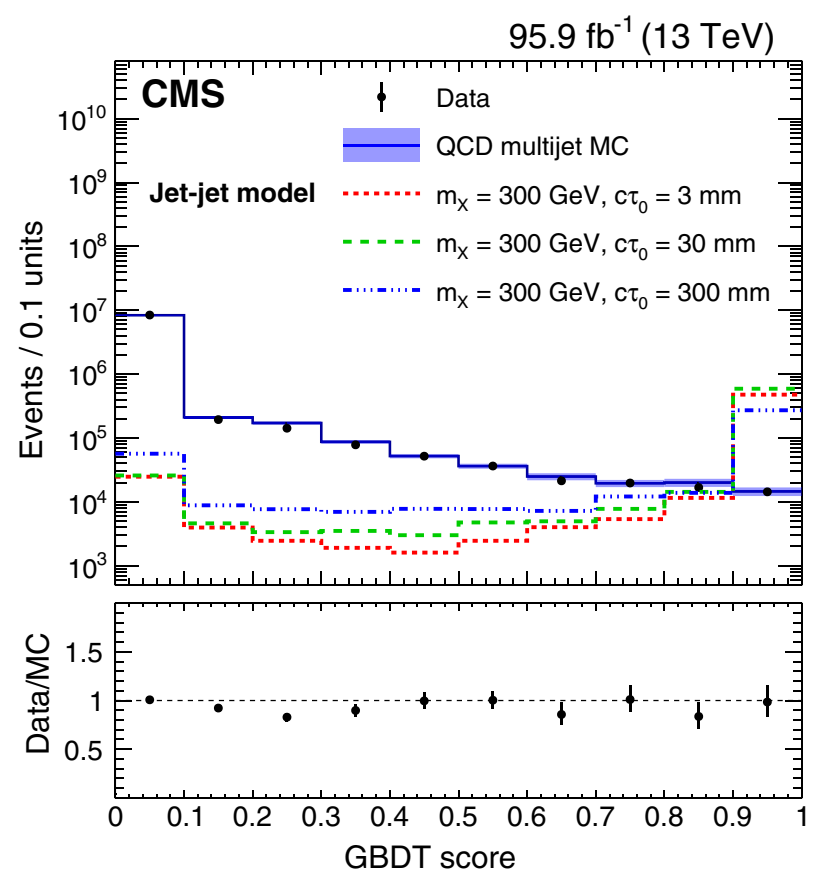

FIG. 4. The distributions of the GBDT output score for data, simulated QCD multijet events, and simulated signal events. Data and simulated events are selected with the displaced-jet triggers and with the offline $H_{\mathrm{T}}$, jets $p_{\mathrm{T}}$, and $\eta$ selections applied. For a given event, if there is more than one SV candidate being reconstructed, the one with the largest vertex track multiplicity is chosen. If the track multiplicities are the same, the one with the smallest $\chi^{2} / \mathrm{n}_{\text {dof }}$ is chosen. The lower panel shows the ratio between the data and the simulated QCD multijet events. The blue shaded error bands and vertical bars represent the statistical uncertainties. Three benchmark signal distributions are shown (dashed lines) for the jet-jet model with $m_{\mathrm{X}}=300 \mathrm{GeV}$ and varying $c \tau_{0}$. For visualization purposes, each signal process is given a cross section corresponding to $10^{6}$ events produced in the analyzed data sample. The signal events shown in this plot are not used in the GBDT training. signatures of other LLP models with different final-state topologies, since we have explicitly chosen the input variables to make the GBDT as model-independent as possible.

In addition to the GBDT score $g$, we use another variable $N_{\text {tracks }}^{3 \mathrm{D}}$ in the final event selection, which is the number of $3 \mathrm{D}$ prompt tracks in a single jet, where the 3D prompt tracks are the tracks that have 3D impact parameters with respect to the leading PV smaller than $0.3 \mathrm{~mm}$.

If more than one dijet candidate passes the preselection criteria described in Sec. IV, the one with the largest GBDT score is selected. If the GBDT scores are the same, the one with the smallest $\mathrm{SV} \chi^{2} / \mathrm{n}_{\mathrm{dof}}$ is selected. In the final signal region, the candidate is further required to pass three final selection criteria, which are

(i) Selection 1: for the leading jet in $p_{\mathrm{T}}, N_{\text {tracks }}^{3 \mathrm{D}}$ is smaller than 3;

(ii) Selection 2: for the subleading jet, $N_{\text {tracks }}^{3 \mathrm{D}}$ is smaller than 3 ; and

(iii) Selection 3: the GBDT score $g$ is larger than 0.988 . The numerical values for the selection criteria are chosen by optimizing the discovery potential of 5 standard deviations based on the Punzi significance [86] for the jet-jet model and the $\tilde{g} \rightarrow g \tilde{G}$ model across different LLP masses and lifetimes. The chosen models encompass the displaceddijet and displaced-single-jet signatures, with $m_{\mathrm{X}}=100$, 300 , and $1000 \mathrm{GeV}$ for the jet-jet model, and with $m_{\tilde{g}}=600,1000$, and $1600 \mathrm{GeV}$ for the $\tilde{g} \rightarrow g \tilde{G}$ model, while $c \tau_{0}$ is taken to be $1,10,100$, and $1000 \mathrm{~mm}$. Thus there are 24 signal points considered in total for the selection optimization.

Based on the three selection criteria, we can define eight nonoverlapping regions $\mathrm{A}-\mathrm{H}$, which include the final signal region. The event counts in different regions are $N_{f f f}, N_{p f f}, \cdots$, and $N_{p p p}$, for regions $\mathrm{A}, \mathrm{B}, \cdots$, and $\mathrm{H}$, respectively, as shown in Table II. The region $\mathrm{H}$ is the region where the events pass all the three selection criteria, and thus is the final signal region. Events in the remaining regions (A-G) fail one or more of the three selection criteria. Since the three selection criteria have little correlation between them for the background events, a property

TABLE II. The definitions of the different regions used in the background estimation.

\begin{tabular}{lcccc}
\hline \hline Region & Selection 1 & Selection 2 & Selection 3 & Event count \\
\hline A & Fail & Fail & Fail & $N_{f f f}$ \\
B & Pass & Fail & Fail & $N_{p f f}$ \\
C & Fail & Pass & Fail & $N_{f p f}$ \\
D & Fail & Fail & Pass & $N_{f f p}$ \\
E & Fail & Pass & Pass & $N_{f p p}$ \\
F & Pass & Fail & Pass & $N_{p f p}$ \\
G & Pass & Pass & Fail & $N_{p p f}$ \\
H & Pass & Pass & Pass & $N_{p p p}$ \\
\hline \hline
\end{tabular}


that has been verified with simulated QCD multijet events, the background yield in the signal region $\mathrm{H}$ can be estimated by different ratios of event counts in regions $\mathrm{A}-\mathrm{G}$, where the ratio $b_{\text {nominal }}=N_{p p f}\left(N_{f f p}+N_{f p p}+\right.$ $\left.N_{p f p}\right) /\left(N_{f f f}+N_{p f f}+N_{f p f}\right)$ uses the fraction of events passing to those failing the GBDT selection (Selection 3) and is taken as the central value of the predicted background yields. Three additional ratios are computed using the events failing either one of the first two selections:

(i) Cross-check

$$
\text { 1: } N_{p p f}\left(N_{f f p}+N_{f p p}\right) /\left(N_{f f f}+N_{f p f}\right)
$$

(ii) Cross-check

$$
\text { 2: } N_{p p f}\left(N_{f f p}+N_{p f p}\right) /\left(N_{f f f}+N_{p f f}\right)
$$

(iii) Cross-check

$$
\text { 3: } N_{p p f}\left(N_{f p p}+N_{p f p}\right) /\left(N_{f p f}+N_{p f f}\right) \text {. }
$$

These cross-checks provide an important test of the robustness of the background prediction and the assumption that the three selection criteria are minimally correlated. Differences between the predictions obtained with the nominal ratio $b_{\text {nominal }}$ and the cross-checks are used to estimate the systematic uncertainties in the background prediction.

The method described above can be generalized to predict the background yield in arbitrary intervals of the GBDT score $g$, where the first two selections are also satisfied. In this way we can verify our background prediction method in the different bins of GBDT score $g$. The background prediction method is first tested with simulated QCD multijet events and simulated signal events, and is found to be robust both with and without signal contaminations. We then test the background prediction in data, for which we define a control region by inverting the selection on $\epsilon$, requiring $\epsilon$ to be smaller than 0.15 . In order to improve the statistical precision, we remove the selection requirement that uses the NI-veto map, although the background prediction method is still robust with the presence of the NI-veto map. The predicted background yields and observed events in the control region are shown in Fig. 5, which shows a good agreement between the predicted and observed yields. The predicted background yields in the different bins of GBDT score are correlated, since the events that are used for background predictions in lower bins are also used in the background predictions in higher bins.

\section{SYSTEMATIC UNCERTAINTIES}

The systematic uncertainty in the background prediction is taken to be the largest deviation of the three cross checks from the nominal prediction $b_{\text {nominal }}$, and is found to be $52 \%$ in the final signal region where the GBDT score $g$ is larger than 0.988 .

The systematic uncertainties in the integrated luminosity for $13 \mathrm{TeV} p p$ collision data are $2.3 \%$ and $2.5 \%$ for the 2017 and $2018[87,88]$ data taking periods, respectively,

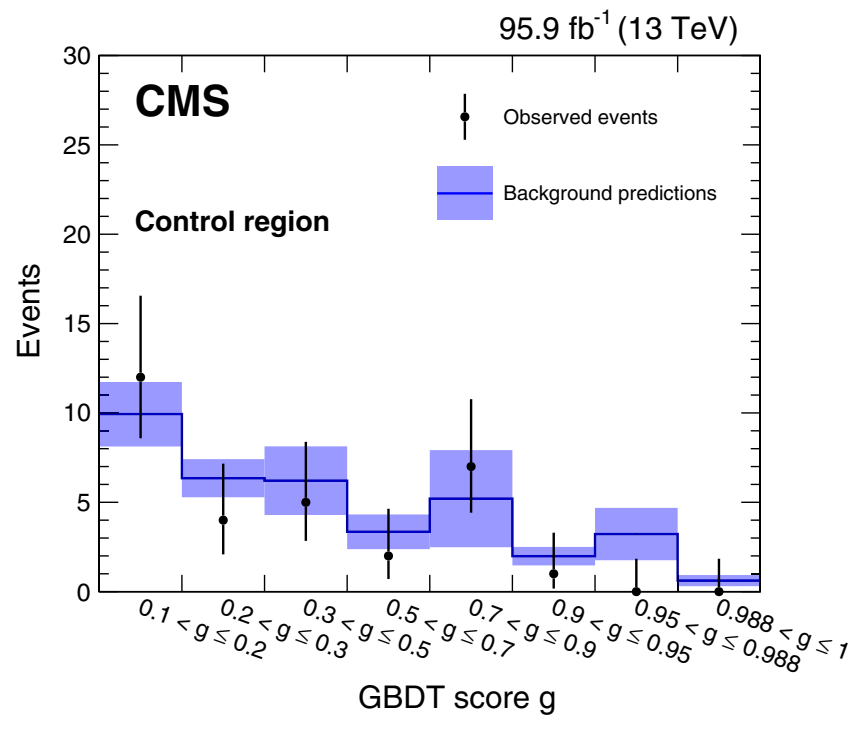

FIG. 5. The predicted background yields and the numbers of observed events in the control region, for different bins of the GBDT scores. The background predictions in different bins are correlated, since the events that are used for background predictions in lower bins are also used in the background predictions in higher bins. The error bands for the predictions represent statistical uncertainties and systematic uncertainties added in quadrature. The error bars for the observed events represent statistical uncertainties, assuming Poisson statistics.

and are modeled as uncorrelated nuisance parameters between the years.

The systematic uncertainty in the signal yields arising from the pileup modeling is estimated by varying the inelastic $p p$ cross section [89] used in the pileup distribution determination by $4.6 \%$. The resulting variations of the signal yields are generally smaller than $1 \%$ and are well within the statistical fluctuations, therefore the impact of the pileup modeling is negligible and no corresponding systematic uncertainty is assigned.

The systematic uncertainty in the signal yields due to the online $H_{\mathrm{T}}$ requirements of the displaced-jet triggers is estimated by comparing the efficiency of the online $H_{\mathrm{T}}$ requirements measured in data with the one measured in the QCD multijet MC sample. The efficiencies are measured using the events collected with an isolated single-muon trigger. The discrepancies between the measurements in data and $\mathrm{MC}$ simulation are parameterized as functions of offline $H_{\mathrm{T}}$, and corresponding corrections are applied to the simulated signal events. The signal yields are then recalculated for different masses and mean proper decay lengths. The largest correction in the signal yield for a given model is taken as the corresponding systematic uncertainty, and is found to be smaller than $2 \%$.

The systematic uncertainty in the signal yields due to the online jet $p_{\mathrm{T}}$ requirements of the displaced-jet triggers is estimated by measuring the per-jet efficiencies of the online jet $p_{\mathrm{T}}$ requirements in data and in the QCD multijet MC 
sample, using events collected with a prescaled $H_{\mathrm{T}}$ trigger that requires $H_{\mathrm{T}}>425 \mathrm{GeV}$. Corrections for the discrepancies between the measurements in data and MC simulation are applied to the simulated signal events, and the signal yields are recalculated. The correction is more significant for low-mass LLPs (with $m \sim 50 \mathrm{GeV}$ ), and is smaller than $8 \%$, which is taken as the corresponding systematic uncertainty.

To estimate the systematic uncertainty due to the online tracking requirements of the displaced-jet triggers, we measure the per-jet efficiencies of the online tracking requirements as functions of the number of prompt tracks and displaced tracks, using events collected with the prescaled $H_{\mathrm{T}}$ trigger. The efficiencies obtained in data are found to be consistent with the efficiencies obtained in MC simulations. Therefore no corresponding systematic uncertainty is assigned.

To estimate the impact of the possible mismodeling of the GBDT score in MC simulation on the signal yields, we compare the distribution of the GBDT score in simulated QCD multijet events with the distribution measured in data, using events collected with the prescaled $H_{\mathrm{T}}$ trigger. The discrepancies between data and MC simulation are taken into account by varying the GBDT scores in the simulated samples by the same magnitude. The largest variation in the signal efficiency for a given model is taken as the corresponding systematic uncertainty in signal yields, and is found to be in the range of $4 \%-15 \%$.

Similarly, the uncertainty in the signal yields due to impact parameter modeling is estimated by comparing the distribution of the impact parameters in simulated QCD multijet events with the distribution measured in data, also using events collected with the prescaled $H_{\mathrm{T}}$ trigger. The impact parameters in simulated QCD multijet events are varied until the discrepancies between data and MC simulation are covered by the variation. The impact parameters in the simulated signal samples are then varied by the same magnitude. The largest variation in the signal efficiency for a given model is taken as the corresponding systematic uncertainty, and is found to be in the range of $8 \%-18 \%$.

The impact of the jet energy scale uncertainty on the signal yields is estimated by varying the jet energy and $p_{\mathrm{T}}$ by one standard deviation of the jet energy scale uncertainty [53]. The variations of the signal efficiencies are smaller than $3 \%$, which is taken as the corresponding systematic uncertainty.

The impact of the PDF uncertainty on the signal acceptance is estimated by reweighting the simulated signal events with NNPDF, CT14 [90] and MMHT14 [91] PDF sets, and their associated uncertainty sets [92,93], following the PDF4LHC recommendation [92]. The uncertainty in signal efficiency for a given signal model is quantified by comparing the efficiencies calculated with alternative PDF sets and the ones with the nominal NNPDF set, and is found to be in the range of $4-6 \%$.
TABLE III. Summary of the systematic uncertainties in the signal yields.

\begin{tabular}{lc}
\hline \hline Source & Uncertainties $(\%)$ \\
\hline Integrated luminosity & $2.3-2.5$ \\
Online $H_{\mathrm{T}}$ requirement & $0-2$ \\
Online jet $p_{\mathrm{T}}$ requirement & $0-8$ \\
Offline vertexing & $4-15$ \\
Track impact parameter modeling & $8-18$ \\
Jet energy scale & $0-3$ \\
PDF & $4-6$ \\
Primary vertex selection & $8-15$ \\
Total & $17-25$ \\
\hline \hline
\end{tabular}

The uncertainty in the signal yields due to the selection of the PV is estimated by replacing the leading PV with the subleading PV when calculating impact parameters and vertex displacement. The largest variation of the signal efficiency for a given signal model is taken as the corresponding systematic uncertainty, and is found to be in the range of $8-15 \%$.

The various systematic uncertainties in the signal yields are summarized in Table III.

\section{RESULTS}

\section{A. Data in the signal region}

The predicted background yields and the numbers of observed events in different GBDT ranges, after all the preselection criteria are applied, are shown in Fig. 6, with $N_{\text {tracks }}^{3 \mathrm{D}}$ smaller than 3 for both jets. The final signal region is defined by a GBDT score larger than 0.988 , and the predicted background yield is $0.75 \pm 0.44$ (stat) \pm 0.39 (syst) events in the data samples collected in 2017 and 2018. After all the preselection criteria are applied, the efficiencies for signal events to have at least one SV/dijet candidate satisfying GBDT $>0.988$ are generally above 80-90\% for the different LLP models considered with $m_{\mathrm{LLP}} \gtrsim 300 \mathrm{GeV}$ and $1 \lesssim c \tau_{0} \lesssim 1000 \mathrm{~mm}$, while the background rate has been reduced by a factor of around $3 \times 10^{3}$ by the GBDT selection.

Event yields in data after different selection requirements have been applied are shown in Table IV. We observe one event in the final signal region, which is consistent with the predicted background yield. The observed event has an offline $H_{\mathrm{T}}$ of $570 \mathrm{GeV}$, and an SV candidate with an $L_{x y}$ of $\approx 26 \mathrm{~cm}$ and 8 associated tracks. The position of the $\mathrm{SV}$ is close to one of the silicon strip layers, and was likely produced by NIs with the silicon strip detector.

\section{B. Interpretation of the results}

The signal yields in the final signal region are used to set limits on a variety of models. The signal efficiencies for representative signal points in different models can be found in Tables V-XIII and Fig. 15 of Appendix. The 


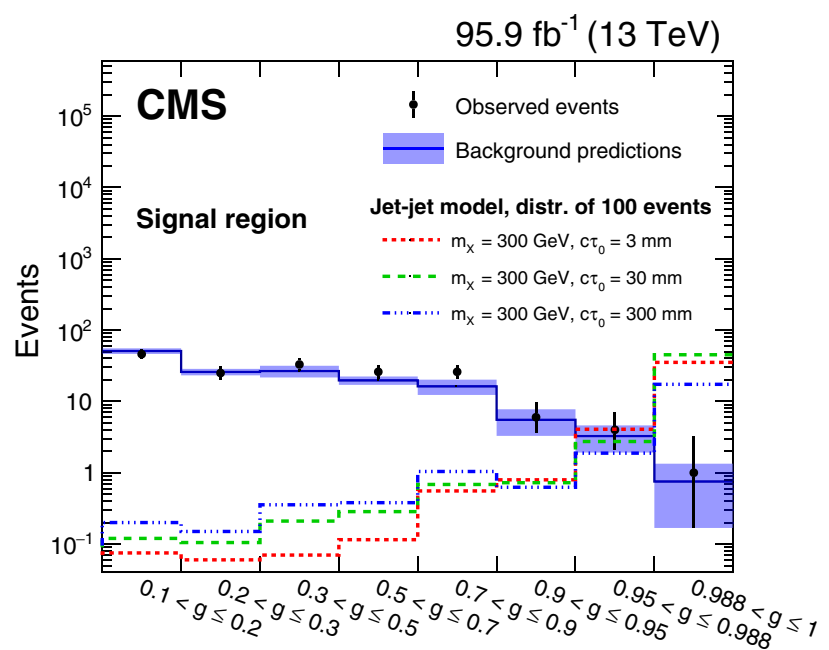

GBDT score $\mathrm{g}$

FIG. 6. The predicted background yields and the number of observed events for the data in the signal region, with $N_{\text {tracks }}^{3 \mathrm{D}}$ smaller than 3 for both jets, shown for different bins of the GBDT scores. The background predictions in different bins are correlated, since the events that are used for background predictions in lower bins are also used in the background predictions in higher bins. For comparison, three benchmark signal points are also shown (dashed lines) for the jet-jet model with $m_{\mathrm{X}}=300 \mathrm{GeV}$ and different lifetimes. For visualization purposes, each signal process is given a cross section that yields 100 events produced in the analyzed data sample.

results obtained with the 2017-2018 analysis are further combined in this paper with the results from the displacedjets search using the $p p$ collision data collected with the CMS experiment in 2016 [40], for which the systematic uncertainties arising from the same source are taken to be fully correlated, while the other systematic uncertainties and the statistical uncertainties in signal yields or expected background yields are taken to be uncorrelated. The total integrated luminosity is $132 \mathrm{fb}^{-1}$. For the models that were not studied in the 2016 displaced-jets search, we have produced additional signal MC samples following the 2016 run condition of the CMS detector. We then process these samples with the reconstruction and selection procedures implemented in the 2016 search to compute the additional signal yields and systematic uncertainties for the 2016 data that are used in the combination.

Upper limits on the cross section for a given model are presented with different masses and lifetimes by computing the $95 \%$ confidence level (C.L.) associated with each signal point using the $\mathrm{CL}_{\mathrm{s}}$ prescription [94-97], for which an LHC-style profile likelihood ratio $[96,97]$ is taken as the test statistic. Systematic uncertainties are incorporated through the use of nuisance parameters treated according to the frequentist paradigm. The asymptotic approximation [96] is used for the calculation of the $\mathrm{CL}_{\mathrm{s}}$ values, which have been verified with full-frequentist results for representative signal points. Since the background yields of this search are small, the impact of the associated statistical or systematic uncertainties on the upper limits are also small.

The expected and observed upper limits on the pair production cross section for the jet-jet model at different values of $c \tau_{0}$ and LLP mass $m_{\mathrm{X}}$ are shown in Fig. 7, where a branching fraction of $100 \%$ for $\mathrm{X}$ to decay into a quarkantiquark pair is assumed. For a fixed LLP mass $m_{\mathrm{X}}$, the limits are most restrictive for $c \tau_{0}$ between 3 and $300 \mathrm{~mm}$. For smaller $c \tau_{0}$, the limits become less stringent, because we only select displaced tracks to reconstruct SVs and we veto dijet candidates with a large number of prompt tracks. The limits also become less restrictive for larger $c \tau_{0}$ $\left(c \tau_{0}>300 \mathrm{~mm}\right)$, because the tracking efficiency becomes worse with large displacement of the SV. For high-mass

TABLE IV. Event yields after different selection requirements have been applied for data collected in 2017 and 2018. Signal efficiencies for the jet-jet model with $m_{\mathrm{X}}=1000 \mathrm{GeV}$ and different $c \tau_{0}$ are also shown for comparison. Selection requirements are cumulative from the first row to the last.

\begin{tabular}{|c|c|c|c|c|}
\hline \multirow[b]{2}{*}{ Selections } & \multirow[b]{2}{*}{ Observed events } & \multicolumn{3}{|c|}{$\begin{array}{c}\text { Signal efficiency }(\%) \\
m_{\mathrm{X}}=1000 \mathrm{GeV} \\
c \tau_{0}\end{array}$} \\
\hline & & $3 \mathrm{~mm}$ & $30 \mathrm{~mm}$ & $300 \mathrm{~mm}$ \\
\hline Displaced-jet triggers, offline $H_{\mathrm{T}}$ selections & 17758640 & 69.4 & 91.2 & 80.5 \\
\hline Offline jet $p_{\mathrm{T}}$ and $\eta$ selections, vertex $\chi^{2} / \mathrm{n}_{\mathrm{dof}}<5.0$ & 8387775 & 68.9 & 90.7 & 73.5 \\
\hline Vertex $p_{\mathrm{T}}>8 \mathrm{GeV}$ & 3794960 & 68.2 & 90.3 & 69.4 \\
\hline Vertex invariant mass $>4 \mathrm{GeV}$ & 1129531 & 66.5 & 89.3 & 61.6 \\
\hline Second largest $\operatorname{Sig}\left[\mathrm{IP}_{2 \mathrm{D}}\right]>15$ & 422449 & 66.0 & 89.0 & 60.9 \\
\hline Charged energy fraction from the SV $\epsilon>0.15$ & 93873 & 64.3 & 87.6 & 58.4 \\
\hline Energy fraction from the PVs $\zeta<0.20$ & 15891 & 63.6 & 86.9 & 57.9 \\
\hline Veto using the NI-veto map & 13721 & 63.6 & 84.9 & 55.4 \\
\hline$N_{\text {tracks }}^{3 \mathrm{D}}<3$ for each jet & 2753 & 54.6 & 76.4 & 48.4 \\
\hline GBDT $>0.988$ & 1 & 51.5 & 73.5 & 42.5 \\
\hline
\end{tabular}



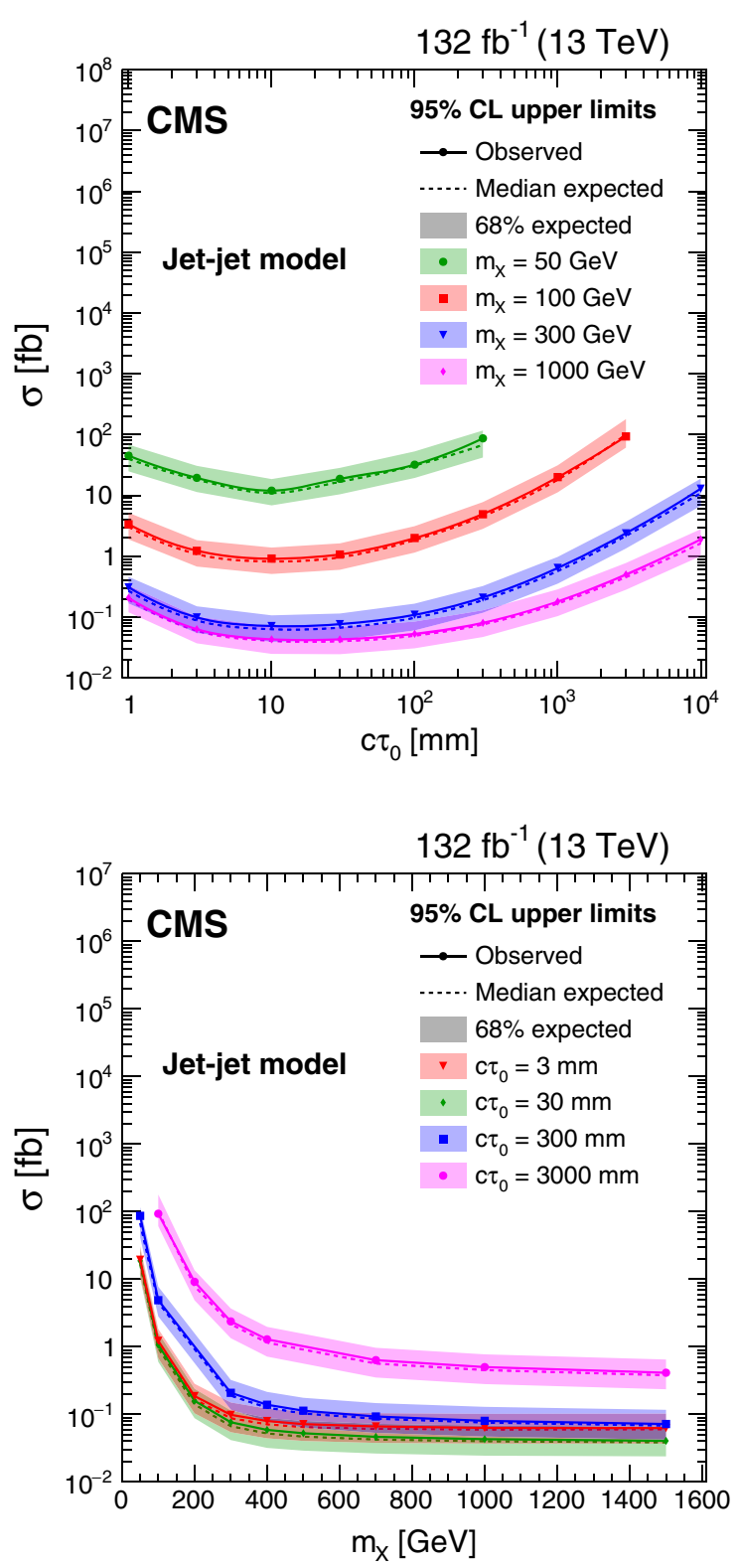

FIG. 7. The $95 \%$ C.L. upper limits on the pair production cross section of the LLP X, where a $100 \%$ branching fraction for X to decay to a quark-antiquark pair is assumed. Upper: the upper limits as functions of $c \tau_{0}$ for different masses. Lower: the upper limits as functions of the particle mass for different $c \tau_{0}$. The solid (dashed) curves show the observed (median expected) limits. The shaded bands indicate the regions containing $68 \%$ of the distributions of the limits expected under the background-only hypothesis.

LLPs $\left(m_{\mathrm{X}}>500 \mathrm{GeV}\right)$, pair production cross sections larger than $0.07 \mathrm{fb}$ are excluded for $c \tau_{0}$ between 2 and $250 \mathrm{~mm}$. The lowest pair production cross section excluded is $0.04 \mathrm{fb}$, at $c \tau_{0}=30 \mathrm{~mm}$ and $m_{\mathrm{X}}>1000 \mathrm{GeV}$.

Figure 8 presents the expected and observed upper limits on the branching fraction of the SM-like Higgs boson to decay to two long-lived scalar particles $\mathrm{S}$, each of which decays $100 \%$ of times to a quark-antiquark pair of a specific flavor. The upper limits on the branching fraction are
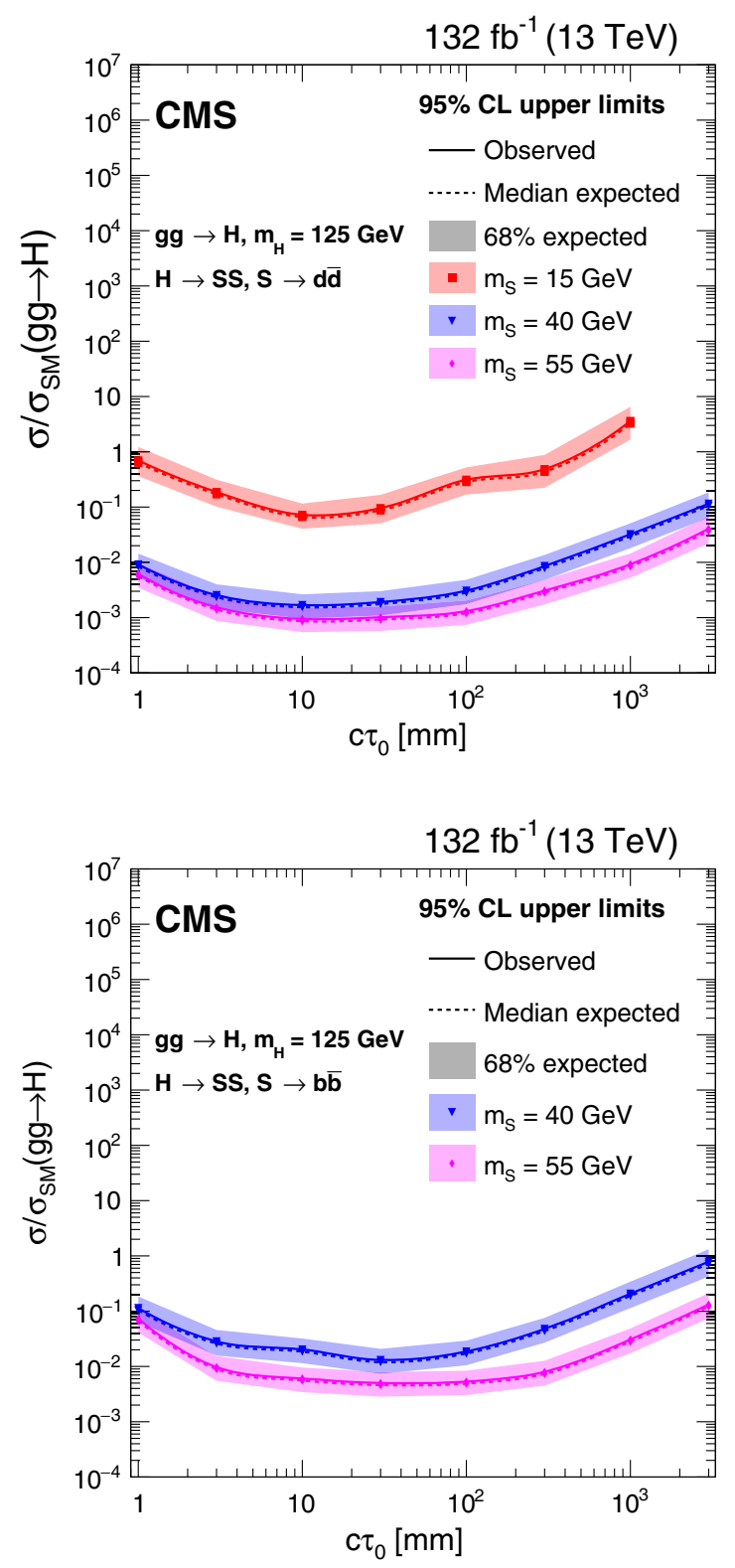

FIG. 8. The expected and observed 95\% C.L. upper limits on the branching fraction of the SM-like Higgs boson to decay to two long-lived scalar particles, assuming the gluon-gluon fusion Higgs boson production cross section of $49 \mathrm{pb}$ at $13 \mathrm{TeV}$ with $m_{H}=125 \mathrm{GeV}$, shown at different masses and $c \tau_{0}$ for the scalar particle S. Upper: the upper limits when each scalar particle decays to a down quark-antiquark pair. Lower: the upper limits when each scalar particle decays to a bottom quark-antiquark pair. The solid (dashed) curves represent the observed (median expected) limits. The shaded bands represent the regions containing $68 \%$ of the distributions of the expected limits under the background-only hypothesis.

calculated assuming the gluon-gluon fusion production cross section of a $125 \mathrm{GeV}$ Higgs boson at $13 \mathrm{TeV}$ [60]. When the long-lived scalar particle decays to a light-flavor quark-antiquark pair, branching fractions larger than $1 \%$ are excluded for $c \tau_{0}$ between 1 and $340 \mathrm{~mm}$ with 
$m_{\mathrm{S}} \geq 40 \mathrm{GeV}$. When the long-lived scalar particle decays to a bottom quark-antiquark pair, branching fractions larger than $10 \%$ are excluded for $c \tau_{0}$ between 1 and $530 \mathrm{~mm}$ with $m_{\mathrm{S}} \geq 40 \mathrm{GeV}$. These are the most stringent limits to date on this model for $c \tau_{0}$ between 1 and $1000 \mathrm{~mm}$. For $m_{\mathrm{S}}=15 \mathrm{GeV}$, where the track multiplicity of the SV is small, and the tracks are collimated due to the boost of $\mathrm{S}$, the limits become worse. The limits are also worse for the case where the scalar particle decays to a bottom quarkantiquark pair, because the decays of $b$ hadrons can produce tertiary vertices, which can be missed by the SV reconstruction we deploy in this search.

The expected and observed upper limits on the pair production cross section of long-lived gluinos in the GMSB $\tilde{g} \rightarrow g \tilde{G}$ model are shown in Fig. 9, where a branching fraction of $100 \%$ for the gluino to decay into a gluon and a gravitino is assumed. Since we do not require the reconstructed SV to have associated tracks from both jets, the two separate displaced single jets produced by the decays of the two long-lived gluinos in the $\tilde{g} \rightarrow g \tilde{G}$ model can be paired together and pass the selections, therefore the search is sensitive to the models with similar signatures. When the gluino mass is $2400 \mathrm{GeV}$, signal efficiencies are around 21 , 53, and $41 \%$ in the 2017 and 2018 analysis for $c \tau_{0}=3,30$, and $300 \mathrm{~mm}$, respectively. With the data samples collected in 2016-2018, gluino pair production cross sections larger than $0.1 \mathrm{fb}$ are excluded for $c \tau_{0}$ between 7 and $600 \mathrm{~mm}$ at $m_{\tilde{g}}=2400 \mathrm{GeV}$. We then compute the upper limits on the gluino mass for different $c \tau_{0}$ according to the upper limits on the pair production cross section, and a calculation at nextto-next-to-leading logarithmic precision matched to the approximated next-to-next-to-leading order predictions $\left(\mathrm{NNLO}_{\text {approx }}+\mathrm{NNLL}\right)$ of the gluino pair production cross section at $\sqrt{s}=13 \mathrm{TeV}$ [98-103]. Gluino masses up to $2450 \mathrm{GeV}$ are excluded for $c \tau_{0}$ between 6 and $550 \mathrm{~mm}$. The largest gluino mass excluded is $2560 \mathrm{GeV}$ with a $c \tau_{0}$ of $30 \mathrm{~mm}$. These limits are the most restrictive to date on this model for $c \tau_{0}$ between 1 and $1000 \mathrm{~mm}$.

Figure 10 shows the expected and observed upper limits on the pair production cross section of the long-lived gluinos in the minisplit $\tilde{g} \rightarrow q \bar{q} \tilde{\chi}_{1}^{0}$ model, assuming a branching fraction of $100 \%$ for the gluino to decay into a quark-antiquark pair and the lightest neutralino. The neutralino mass is assumed to be $100 \mathrm{GeV}$. When the gluino mass is $2400 \mathrm{GeV}$, signal efficiencies are around 31 , 69, and 51\% in the 2017 and 2018 analysis for $c \tau_{0}=3,30$, and $300 \mathrm{~mm}$, respectively. With the data samples collected in 2016-2018, gluino pair production cross sections larger than $0.1 \mathrm{fb}$ are excluded for proper decay lengths between 3 and $900 \mathrm{~mm}$. The upper limits on the pair production cross sections are then translated into upper limits on the gluino mass for different $c \tau_{0}$, based on the $\mathrm{NNLO}_{\text {approx }}+\mathrm{NNLL}$ gluino pair production cross sections. Gluino masses up to $2500 \mathrm{GeV}$ are excluded for $c \tau_{0}$ between 7 and $360 \mathrm{~mm}$. The largest gluino mass excluded is $2610 \mathrm{GeV}$ with a $c \tau_{0}$ of
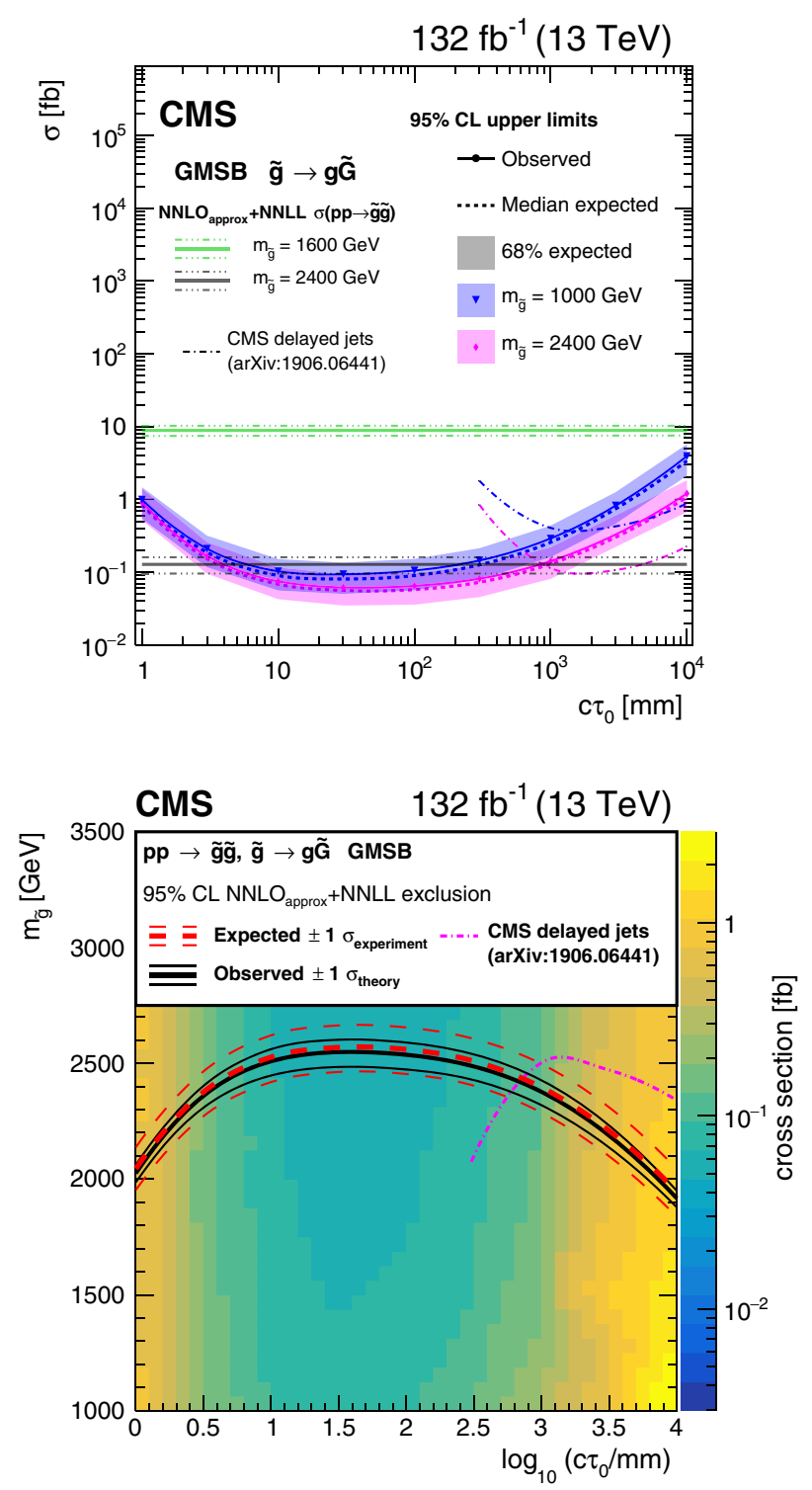

FIG. 9. Upper: the 95\% C.L. upper limits on the pair production cross section for the long-lived gluinos with $m_{\tilde{g}}=2400$ and $1600 \mathrm{GeV}$, where a $100 \%$ branching fraction for $\tilde{g} \rightarrow g \tilde{G}$ decays is assumed. The $\mathrm{NNLO}_{\text {approx }}+\mathrm{NNLL}$ gluino pair production cross sections for $m_{\tilde{g}}=2400$ and $1600 \mathrm{GeV}$, as well as their variations due to the theoretical uncertainties, are shown as horizontal lines. The solid (dashed) curves show the observed (median expected) limits, and the shaded bands indicate the regions containing $68 \%$ of the distributions of the limits expected under the background-only hypothesis. The observed limits from the CMS search utilizing the timing capabilities of the ECAL system [48] are also shown for comparison. Lower: the $95 \%$ C.L. upper limits on the pair production cross section for the $\tilde{g} \rightarrow g \tilde{G}$ model as a function of the mean proper decay length $c \tau_{0}$ and the gluino mass $m_{\tilde{g}}$. The thick solid black (dashed red) curve shows the observed (median expected) 95\% C.L. limit on the gluino mass as a function of $c \tau_{0}$, assuming the $\mathrm{NNLO}_{\text {approx }}+\mathrm{NNLL}$ cross sections. The thin dashed red curves indicate the region containing $68 \%$ of the distribution of the limits expected under the background-only hypothesis. The thin solid black curves represent the change in the observed limit when the signal cross sections are varied according to their theoretical uncertainties. 

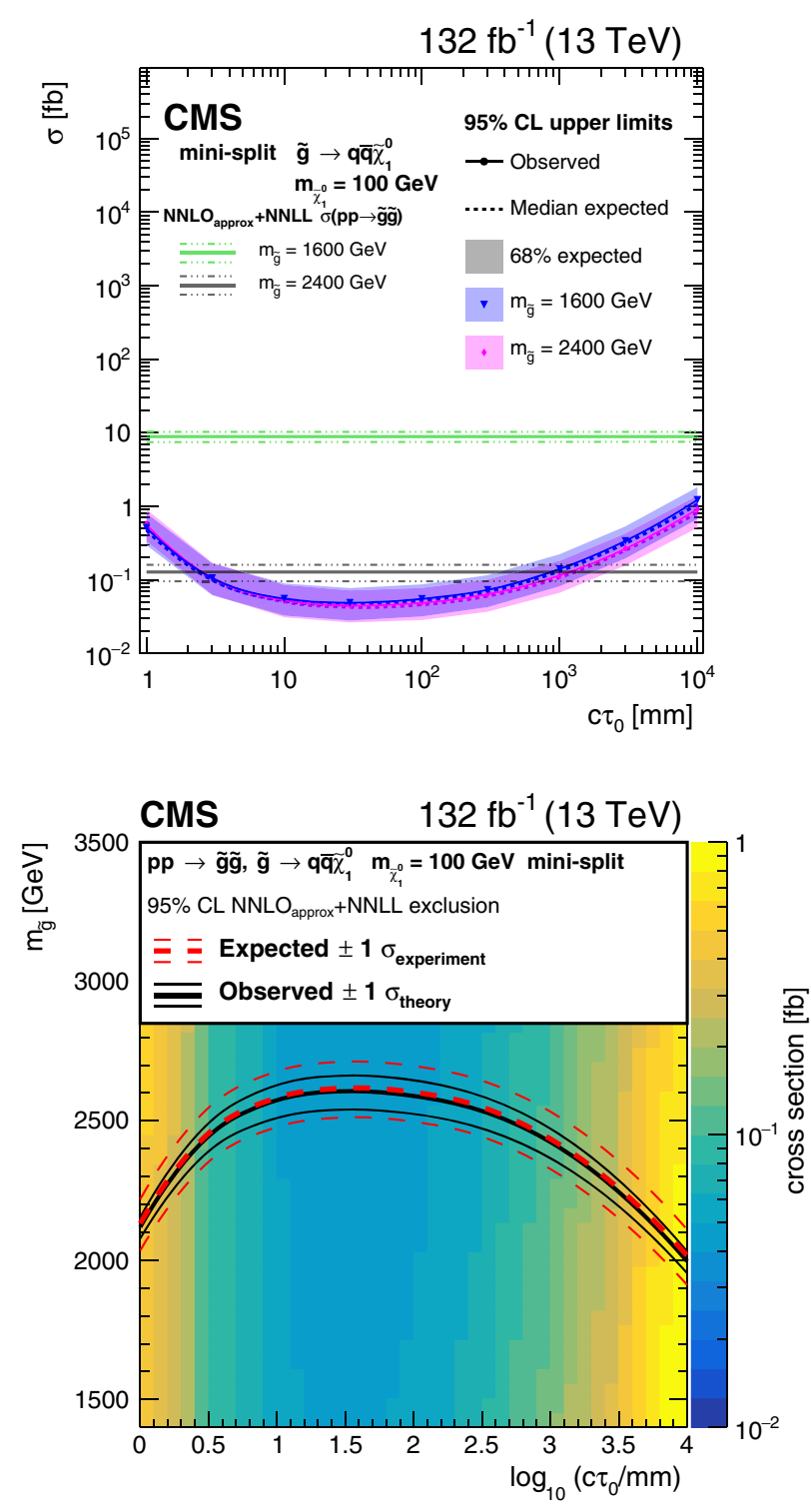

FIG. 10. Upper: the 95\% C.L. upper limits on the pair production cross section for the long-lived gluinos with $m_{\tilde{g}}=$ $2400 \mathrm{GeV}$ and $1600 \mathrm{GeV}$, where a $100 \%$ branching fraction for $\tilde{g} \rightarrow q \bar{q} \tilde{\chi}_{1}^{0}$ decays is assumed. The $\mathrm{NNLO}_{\text {approx }}+$ NNLL gluino pair production cross sections for $m_{\tilde{g}}=2400$ and $1600 \mathrm{GeV}$, as well as their variations due to the theoretical uncertainties, are shown as horizontal lines. The solid (dashed) curves show the observed (median expected) limits, and the shaded bands indicate the regions containing $68 \%$ of the distributions of the limits expected under the background-only hypothesis. Lower: the $95 \%$ C.L. limits on the pair production cross section for the $\tilde{g} \rightarrow q \bar{q} \tilde{\chi}_{1}^{0}$ model as a function of the mean proper decay length $c \tau_{0}$ and the gluino mass $m_{\tilde{g}}$. The thick solid black (dashed red) curve shows the observed (median expected) $95 \%$ C.L. limits on the gluino mass as a function of $c \tau_{0}$, assuming the $\mathrm{NNLO}_{\text {approx }}+\mathrm{NNLL}$ cross sections. The thin dashed red curves indicate the region containing $68 \%$ of the distribution of the limits expected under the background-only hypothesis. The thin solid black curves represent the change in the observed limit when the signal cross sections are varied according to their theoretical uncertainties.
$30 \mathrm{~mm}$. These bounds are the most stringent to date on this model for $c \tau_{0}$ between 10 and $1000 \mathrm{~mm}$.

The expected and observed upper limits on the pair production cross section of the long-lived gluinos in the $\tilde{g} \rightarrow t b s$ model are shown in Fig. 11, where a branching fraction of $100 \%$ for the gluino to decay into top, bottom, and strange quarks is assumed. When the gluino mass is $2400 \mathrm{GeV}$, signal efficiencies are around 41,81 , and $66 \%$ in the 2017 and 2018 analysis for $c \tau_{0}=3,30$, and $300 \mathrm{~mm}$, respectively. With the data samples collected in 20162018, gluino pair production cross sections larger than $0.1 \mathrm{fb}$ are excluded for $c \tau_{0}$ between 3 and $1490 \mathrm{~mm}$ at $m_{\tilde{g}}=2400 \mathrm{GeV}$. We then compute the upper limits on the gluino mass for different $c \tau_{0}$ according to the upper limits on the pair production cross section and the calculation of the $\mathrm{NNLO}_{\text {approx }}+\mathrm{NNLL}$ gluino pair production cross sections. Gluino masses up to $2500 \mathrm{GeV}$ are excluded for $c \tau_{0}$ between 3 and $1000 \mathrm{~mm}$. The largest gluino mass excluded is $2640 \mathrm{GeV}$ with a $c \tau_{0}$ of $30 \mathrm{~mm}$. These limits are the most stringent to date on this model for $c \tau_{0}$ between 30 and $10^{4} \mathrm{~mm}$.

The expected and observed upper limits on the pair production cross section of the long-lived top squarks in the RPV $\tilde{t} \rightarrow b \ell$ model are shown in Fig. 12, where a branching fraction of $100 \%$ for the top squark to decay into a bottom quark and a charged lepton is assumed, with equal branching fractions for $e, \mu$, and $\tau$. When the top squark mass is $1600 \mathrm{GeV}$, signal efficiencies are around 22, 43, and 26\% in the 2017 and 2018 analysis for $c \tau_{0}=3,30$, and $300 \mathrm{~mm}$, respectively. With the data samples collected in 2016-2018, top squark pair production cross sections larger than $0.1 \mathrm{fb}$ are excluded for $c \tau_{0}$ between 8 and $160 \mathrm{~mm}$ at $m_{\tilde{t}}=1600 \mathrm{GeV}$. We then compute the upper limits on the top squark mass for different $c \tau_{0}$ according to the upper limits on the pair production cross section and the calculation of the $\mathrm{NNLO}_{\text {approx }}+\mathrm{NNLL}$ top squark pair production cross sections. Top squark masses up to $1600 \mathrm{GeV}$ are excluded for $c \tau_{0}$ between 5 and $240 \mathrm{~mm}$. The largest top squark mass excluded is $1720 \mathrm{GeV}$ with a $c \tau_{0}$ of $30 \mathrm{~mm}$. These limits are the most stringent to date on this model in the tested $c \tau_{0}$ range.

The expected and observed upper limits on the pair production cross section of the long-lived top squarks in the RPV $\tilde{t} \rightarrow \bar{d} \bar{d}$ model are shown in Fig. 13, where a branching fraction of $100 \%$ for the top squark to decay into a down quark and a charged lepton, with equal branching fractions for $e, \mu$, and $\tau$. When the top squark mass is $1600 \mathrm{GeV}$, signal efficiencies are around 25,48 , and $29 \%$ in the 2017 and 2018 analysis for $c \tau_{0}=3,30$, and $300 \mathrm{~mm}$, respectively. With the data samples collected in 2016-2018, and top squark pair production cross sections larger than $0.1 \mathrm{fb}$ are excluded for $c \tau_{0}$ between 7 and $220 \mathrm{~mm}$. We then compute the upper limits on the top squark mass for different $c \tau_{0}$ according to the upper limits on the pair production cross section and the calculation of 

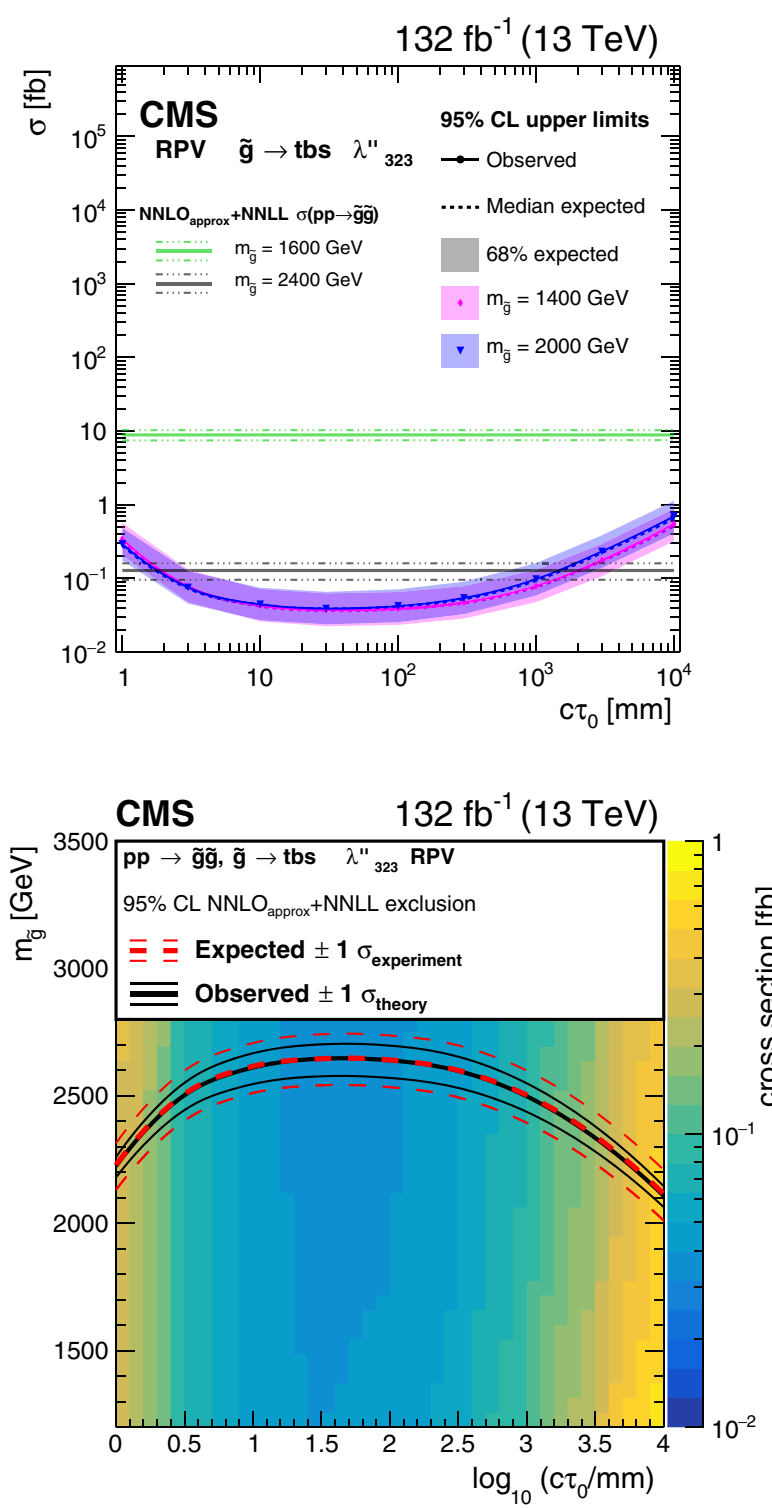

FIG. 11. Upper: the $95 \%$ C.L. upper limits on the pair production cross section for the long-lived gluinos with $m_{\tilde{g}}=$ $2000 \mathrm{GeV}$ and $1400 \mathrm{GeV}$, where a $100 \%$ branching fraction for $\tilde{g} \rightarrow t b s$ decays is assumed. The $\mathrm{NNLO}_{\text {approx }}+\mathrm{NNLL}$ gluino pair production cross sections for $m_{\tilde{g}}=2400$ and $1600 \mathrm{GeV}$, as well as their variations due to the theoretical uncertainties, are shown as horizontal lines. The solid (dashed) curves show the observed (median expected) limits, and the shaded bands indicate the regions containing $68 \%$ of the distributions of the limits expected under the background-only hypothesis. Lower: the $95 \%$ C.L. limits on the pair production cross section for the $\tilde{g} \rightarrow t b s$ model as a function of the mean proper decay length $c \tau_{0}$ and the gluino mass $m_{\tilde{g}}$. The thick solid black (dashed red) curve shows the observed (median expected) $95 \%$ C.L. limits on the gluino mass as a function of $c \tau_{0}$, assuming the $\mathrm{NNLO}_{\text {approx }}+\mathrm{NNLL}$ cross sections. The thin dashed red curves indicate the region containing $68 \%$ of the distribution of the limits expected under the background-only hypothesis. The thin solid black curves represent the change in the observed limit when the signal cross sections are varied according to their theoretical uncertainties.
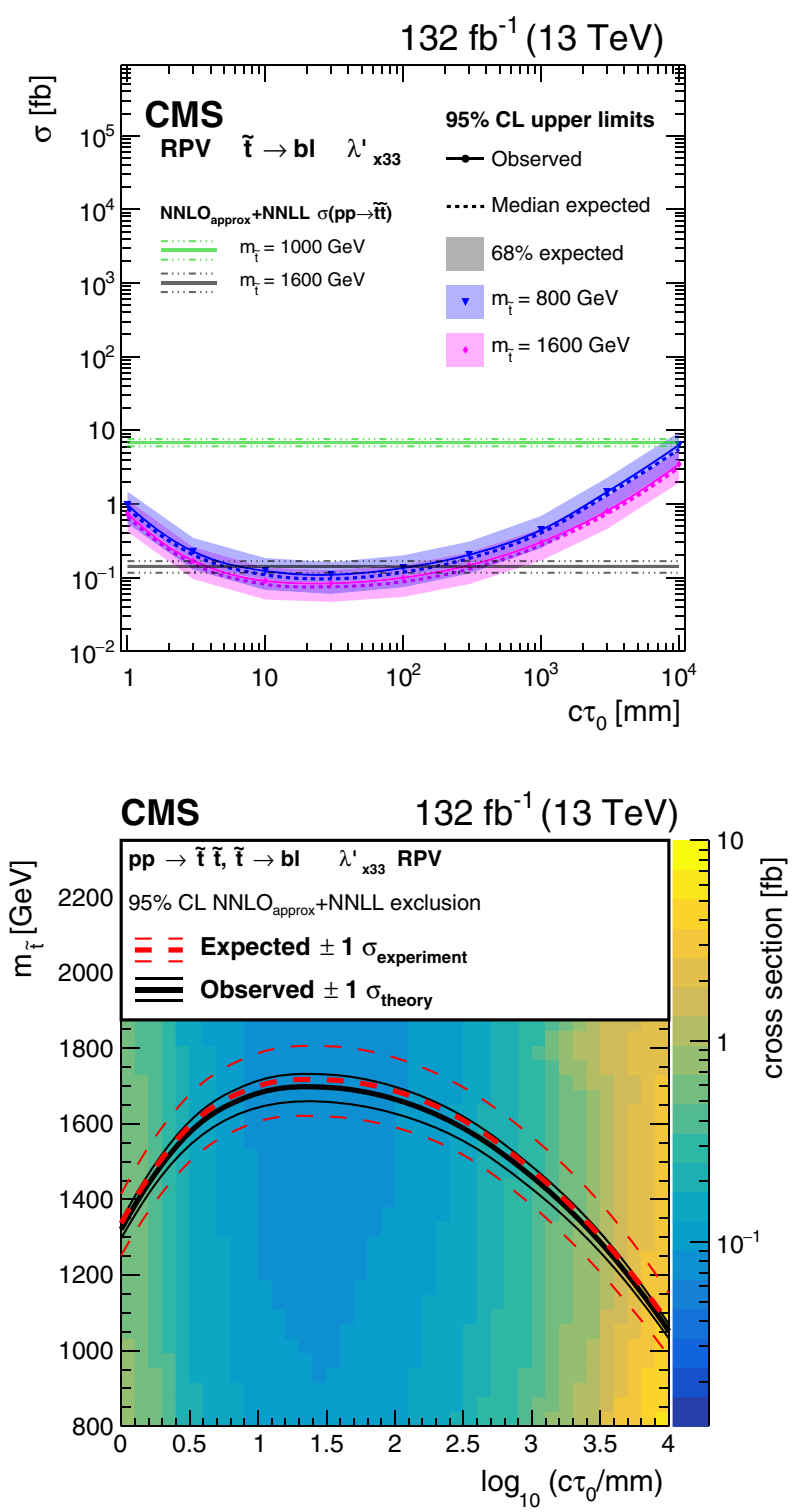

FIG. 12. Upper: the 95\% C.L. upper limits on the pair production cross section for the long-lived top squarks with $m_{\tilde{t}}=1600 \mathrm{GeV}$ and $800 \mathrm{GeV}$, where a $100 \%$ branching fraction for $\tilde{t} \rightarrow b \ell$ decays is assumed, with equal branching fractions for $e, \mu$, and $\tau$. The $\mathrm{NNLO}_{\text {approx }}+\mathrm{NNLL}$ top squark pair production cross sections for $m_{\tilde{t}}=1600$ and $1000 \mathrm{GeV}$, as well as their variations due to the theoretical uncertainties, are shown as horizontal lines. The solid (dashed) curves show the observed (median expected) limits, and the shaded bands indicate the regions containing $68 \%$ of the distributions of the limits expected under the background-only hypothesis. Lower: the $95 \%$ C.L. limits on the pair production cross section for the $\tilde{t} \rightarrow b \ell$ model as a function of the mean proper decay length $c \tau_{0}$ and the top squark mass $m_{\tilde{t}}$. The thick solid black (dashed red) curve shows the observed (median expected) $95 \%$ C.L. limits on the top squark mass as a function of $c \tau_{0}$, assuming the $\mathrm{NNLO}_{\text {approx }}+$ NNLL cross sections. The thin dashed red curves indicate the region containing $68 \%$ of the distribution of the limits expected under the background-only hypothesis. The thin solid black curves represent the change in the observed limit when the signal cross sections are varied according to their theoretical uncertainties. 

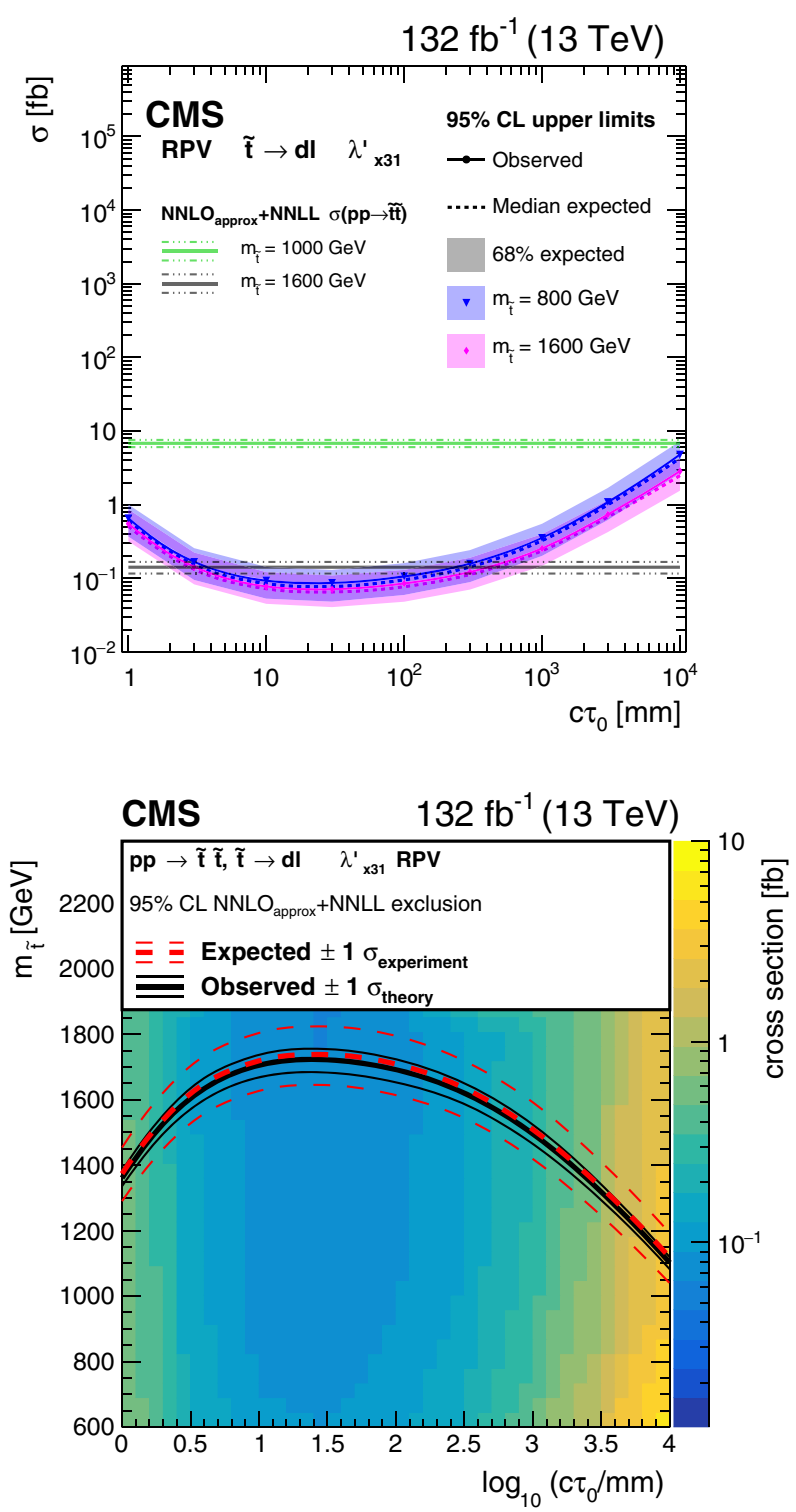

FIG. 13. Upper: the 95\% C.L. upper limits on the pair production cross section for the long-lived top squarks with $m_{\tilde{t}}=1600 \mathrm{GeV}$ and $800 \mathrm{GeV}$, where a $100 \%$ branching fraction for $\tilde{t} \rightarrow d \ell$ decays is assumed, with equal branching fractions for $e, \mu$, and $\tau$. The $\mathrm{NNLO}_{\text {approx }}+\mathrm{NNLL}$ top squark pair production cross sections for $m_{\tilde{t}}=1600$ and $1000 \mathrm{GeV}$, as well as their variations due to the theoretical uncertainties, are shown as horizontal lines. The solid (dashed) curves show the observed (median expected) limits, and the shaded bands indicate the regions containing $68 \%$ of the distributions of the limits expected under the background-only hypothesis. Lower: the $95 \%$ C.L. limits on the pair production cross section for the $\tilde{t} \rightarrow d \ell$ model as a function of the mean proper decay length $c \tau_{0}$ and the top squark mass $m_{\tilde{t}}$. The thick solid black (dashed red) curve shows the observed (median expected) 95\% C.L. limits on the top squark mass as a function of $c \tau_{0}$, assuming the $\mathrm{NNLO}_{\text {approx }}+$ NNLL cross sections. The thin dashed red curves indicate the region containing $68 \%$ of the distribution of the limits expected under the background-only hypothesis. The thin solid black curves represent the change in the observed limit when the signal cross sections are varied according to their theoretical uncertainties.
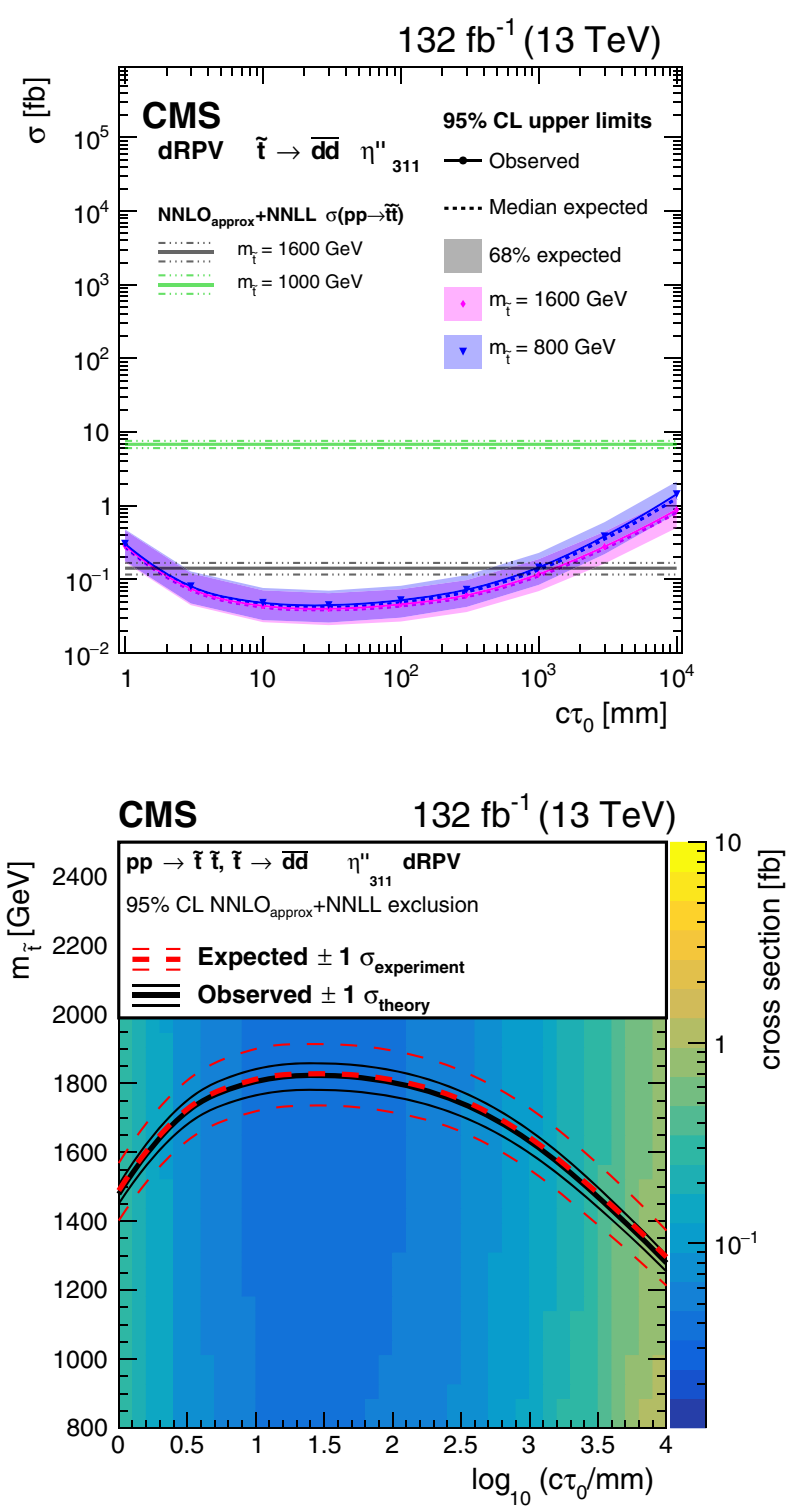

FIG. 14. Upper: the 95\% C.L. upper limits on the pair production cross section for the long-lived top squarks with $m_{\tilde{t}}=1600 \mathrm{GeV}$ and $800 \mathrm{GeV}$, where a $100 \%$ branching fraction for $\tilde{t} \rightarrow \bar{d} \bar{d}$ decays is assumed. The $\mathrm{NNLO}_{\text {approx }}+\mathrm{NNLL}$ top squark pair production cross sections for $m_{\tilde{t}}=1600$ and $1000 \mathrm{GeV}$, as well as their variations due to the theoretical uncertainties, are shown as horizontal lines. The solid (dashed) curves show the observed (median expected) limits, and the shaded bands indicate the regions containing $68 \%$ of the distributions of the limits expected under the background-only hypothesis. Lower: the 95\% C.L. limits on the pair production cross section for the $\tilde{t} \rightarrow \bar{d} \bar{d}$ model as a function of the mean proper decay length $c \tau_{0}$ and the top squark mass $m_{\tilde{t}}$. The thick solid black (dashed red) curve shows the observed (median expected) $95 \%$ C.L. limits on the top squark mass as a function of $c \tau_{0}$, assuming the $\mathrm{NNLO}_{\text {approx }}+$ NNLL cross sections. The thin dashed red curves indicate the region containing $68 \%$ of the distribution of the limits expected under the background-only hypothesis. The thin solid black curves represent the change in the observed limit when the signal cross sections are varied according to their theoretical uncertainties. 
the $\mathrm{NNLO}_{\text {approx }}+\mathrm{NNLL}$ top squark pair production cross sections. Top squark masses up to $1600 \mathrm{GeV}$ are excluded for $c \tau_{0}$ between 3 and $360 \mathrm{~mm}$. The largest top squark mass excluded is $1740 \mathrm{GeV}$ with a $c \tau_{0}$ of $30 \mathrm{~mm}$. These limits are the most restrictive to date on this model in the tested $c \tau_{0}$ range.

The expected and observed upper limits on the pair production cross section of the long-lived top squarks in the dRPV $\tilde{t} \rightarrow \bar{d} \bar{d}$ model are shown in Fig. 14, where a branching fraction of $100 \%$ for the top squark to decay into two down antiquarks is assumed. When the top squark mass is $1600 \mathrm{GeV}$, signal efficiencies are around 43,76 , and $53 \%$ in the 2017 and 2018 analysis for $c \tau_{0}=3,30$, and $300 \mathrm{~mm}$, respectively. With the data samples collected in 2016-2018, top squark pair production cross sections larger than $0.1 \mathrm{fb}$ are excluded for $c \tau_{0}$ between 3 and $820 \mathrm{~mm}$ at $m_{\tilde{t}}=1600 \mathrm{GeV}$. We then compute the upper limits on the top squark mass for different $c \tau_{0}$ according to the upper limits on the pair production cross section and the calculation of the $\mathrm{NNLO}_{\text {approx }}+$ NNLL top squark pair production cross sections. Top squark masses up to $1600 \mathrm{GeV}$ are excluded for $c \tau_{0}$ between 2 and $1320 \mathrm{~mm}$. The largest top squark mass excluded is $1820 \mathrm{GeV}$ with a $c \tau_{0}$ of $30 \mathrm{~mm}$. These bounds are the most stringent to date on this model for $c \tau_{0}$ between 10 and $10^{4} \mathrm{~mm}$.

\section{SUMMARY}

A search has been presented for long-lived particles decaying to jets, using proton-proton collision data collected with the CMS experiment at a center-of-mass energy of $13 \mathrm{TeV}$ in 2017 and 2018. The results are combined with those of a previous CMS search for displaced jets using proton-proton collision data from 2016, accumulating to a total integrated luminosity of $132 \mathrm{fb}^{-1}$. After all selections, one event is observed in the data collected in 2017 and 2018, which is consistent with the predicted background yield. The search is designed to be model independent, and is sensitive to a large number of models predicting displaced-jets signatures with different final-state topologies.

The best current limits are set on a variety of models that have long-lived particles with mean proper decay lengths between $1 \mathrm{~mm}$ and $10 \mathrm{~m}$. All limits are computed at the 95\% confidence level. For a simplified model where pairproduced long-lived neutral particles decay to quarkantiquark pairs, pair production cross sections larger than $0.07 \mathrm{fb}$ are excluded for mean proper decay lengths between 2 and $250 \mathrm{~mm}$ at high mass $\left(m_{\mathrm{X}}>500 \mathrm{GeV}\right)$. For a model where the standard model-like Higgs boson decays to two long-lived scalar particles and each longlived scalar particle decays to a down (bottom) quarkantiquark pair, branching fractions for the exotic Higgs boson decay larger than $1 \%$ (10\%) are excluded for mean proper decay lengths between 1 and $340 \mathrm{~mm}(530 \mathrm{~mm})$ when the scalar particle mass is larger than $40 \mathrm{GeV}$. For a supersymmetric (SUSY) model in the general gauge mediation scenario, where the long-lived gluino decays to a gluon and a lightest SUSY particle, gluino masses up to $2450 \mathrm{GeV}$ are excluded for mean proper decay lengths between 6 and $550 \mathrm{~mm}$. For another SUSY model in the mini-split scenario, where the long-lived gluino can decay to a quark-antiquark pair and the lightest neutralino, gluino masses up to $2500 \mathrm{GeV}$ are excluded for mean proper decay lengths between 7 and $360 \mathrm{~mm}$. An $R$-parity violating (RPV) SUSY model is also tested, where the long-lived gluino can decay to top, bottom, and strange antiquarks, and gluino masses up to $2500 \mathrm{GeV}$ are excluded for mean proper decay lengths between 3 and $1000 \mathrm{~mm}$. Another RPV SUSY model is studied, where the long-lived top squark can decay to a bottom quark and a charged lepton, and top squark masses up to $1600 \mathrm{GeV}$ are excluded for mean proper decay lengths between 5 and $240 \mathrm{~mm}$. For an RPV SUSY model, where the long-lived top squark can decay to a down quark and a charged lepton, top squark masses up to $1600 \mathrm{GeV}$ are excluded for mean proper decay lengths between 3 and $360 \mathrm{~mm}$. Finally, for a dynamicalRPV SUSY model, where the long-lived top squark can decay to two down antiquarks, top squark masses up to $1600 \mathrm{GeV}$ are excluded for mean proper decay lengths between 2 and $1320 \mathrm{~mm}$. These are the most stringent limits to date on these models.

\section{ACKNOWLEDGMENTS}

We congratulate our colleagues in the CERN accelerator departments for the excellent performance of the LHC and thank the technical and administrative staffs at CERN and at other CMS institutes for their contributions to the success of the CMS effort. In addition, we gratefully acknowledge the computing centers and personnel of the Worldwide LHC Computing Grid for delivering so effectively the computing infrastructure essential to our analyses. Finally, we acknowledge the enduring support for the construction and operation of the LHC and the CMS detector provided by the following funding agencies: BMBWF and FWF (Austria); FNRS and FWO (Belgium); CNPq, CAPES, FAPERJ, FAPERGS, and FAPESP (Brazil); MES (Bulgaria); CERN; CAS, MoST, and NSFC (China); COLCIENCIAS (Colombia); MSES and CSF (Croatia); RIF (Cyprus); SENESCYT (Ecuador); MoER, ERC PUT and ERDF (Estonia); Academy of Finland, MEC, and HIP (Finland); CEA and CNRS/IN2P3 (France); BMBF, DFG, and HGF (Germany); GSRT (Greece); NKFIA (Hungary); DAE and DST (India); IPM (Iran); SFI (Ireland); INFN (Italy); MSIP and NRF (Republic of Korea); MES (Latvia); LAS (Lithuania); MOE and UM (Malaysia); BUAP, CINVESTAV, CONACYT, LNS, SEP, and UASLP-FAI (Mexico); MOS (Montenegro); MBIE (New Zealand); PAEC (Pakistan); MSHE and NSC (Poland); FCT (Portugal); JINR (Dubna); MON, RosAtom, RAS, RFBR, and NRC KI (Russia); MESTD (Serbia); SEIDI, CPAN, PCTI, and FEDER (Spain); MOSTR (Sri Lanka); 
Swiss Funding Agencies (Switzerland); MST (Taipei); ThEPCenter, IPST, STAR, and NSTDA (Thailand); TUBITAK and TAEK (Turkey); NASU (Ukraine); STFC (United Kingdom); DOE and NSF (USA). Individuals have received support from the Marie-Curie program and the European Research Council and Horizon 2020 Grant, contracts No. 675440, No. 724704, No. 752730, and No. 765710 (European Union); the Leventis Foundation; the A. P. Sloan Foundation; the Alexander von Humboldt Foundation; the Belgian Federal Science Policy Office; the Fonds pour la Formation à la Recherche dans l'Industrie et dans l'Agriculture (FRIA-Belgium); the Agentschap voor Innovatie door Wetenschap en Technologie (IWTBelgium); the F. R. S.-FNRS and FWO (Belgium) under the "Excellence of Science-EOS"-be.h project n. 30820817; the Beijing Municipal Science \& Technology Commission, No. Z191100007219010; the Ministry of Education, Youth and Sports (MEYS) of the Czech Republic; the Deutsche Forschungsgemeinschaft (DFG) under Germany's Excellence Strategy-EXC 2121 "Quantum Universe"-390833306; the Lendület ("Momentum") Program and the János Bolyai Research Scholarship of the Hungarian Academy of Sciences, the New National Excellence Program ÚNKP, the NKFIA research grants No. 123842, No. 123959, No. 124845,
No. 124850 , No. 125105 , No. 128713 , No. 128786 , and No. 129058 (Hungary); the Council of Science and Industrial Research, India; the HOMING PLUS program of the Foundation for Polish Science, cofinanced from European Union, Regional Development Fund, the Mobility Plus program of the Ministry of Science and Higher Education, the National Science Center (Poland), contracts Harmonia 2014/14/M/ST2/00428, Opus 2014/13/B/ST2/02543, 2014/ 15/B/ST2/03998, and 2015/19/B/ST2/02861, Sonata-bis 2012/07/E/ST2/01406; the National Priorities Research Program by Qatar National Research Fund; the Ministry of Science and Higher Education, project no. 0723-20200041 (Russia); the Tomsk Polytechnic University Competitiveness Enhancement Program; the Programa Estatal de Fomento de la Investigación Científica y Técnica de Excelencia María de Maeztu, Grant No. MDM-2015-0509 and the Programa Severo Ochoa del Principado de Asturias; the Thalis and Aristeia programs cofinanced by EU-ESF and the Greek NSRF; the Rachadapisek Sompot Fund for Postdoctoral Fellowship, Chulalongkorn University and the Chulalongkorn Academic into Its 2nd Century Project Advancement Project (Thailand); the Kavli Foundation; the Nvidia Corporation; the SuperMicro Corporation; the Welch Foundation, Contract C-1845; and the Weston Havens Foundation (USA).

\section{APPENDIX: SIGNAL EFFICIENCIES FOR REPRESENTATIVE SIGNAL POINTS IN DIFFERENT MODELS}

TABLE V. Signal efficiencies for the jet-jet model in the 2017 and 2018 analysis at different mean proper decay lengths $c \tau_{0}$ and different masses $m_{\mathrm{X}}$. Selection requirements are cumulative from the first row to the last for each value of $m_{\mathrm{X}}$. Uncertainties are statistical only.

\begin{tabular}{|c|c|c|c|c|c|c|}
\hline \multirow[b]{2}{*}{ Efficiency (\%) } & \multirow[b]{2}{*}{$m_{\mathrm{X}}(\mathrm{GeV})$} & \multicolumn{5}{|c|}{$c \tau_{0}$} \\
\hline & & $1 \mathrm{~mm}$ & $10 \mathrm{~mm}$ & $30 \mathrm{~mm}$ & $100 \mathrm{~mm}$ & $1000 \mathrm{~mm}$ \\
\hline Trigger & 1000 & $29.47 \pm 0.38$ & $89.98 \pm 0.67$ & $91.16 \pm 0.68$ & $84.41 \pm 0.65$ & $71.72 \pm 0.66$ \\
\hline Preselection & & $22.56 \pm 0.34$ & $85.22 \pm 0.65$ & $84.92 \pm 0.65$ & $73.83 \pm 0.61$ & $27.47 \pm 0.41$ \\
\hline Final selection & & $16.27 \pm 0.29$ & $73.63 \pm 0.61$ & $73.51 \pm 0.61$ & $61.51 \pm 0.55$ & $20.13 \pm 0.35$ \\
\hline Trigger & 300 & $25.05 \pm 0.35$ & $70.50 \pm 0.59$ & $68.19 \pm 0.58$ & $58.97 \pm 0.54$ & $30.22 \pm 0.39$ \\
\hline Preselection & & $17.42 \pm 0.30$ & $59.89 \pm 0.55$ & $55.40 \pm 0.53$ & $42.38 \pm 0.46$ & $9.11 \pm 0.21$ \\
\hline Final selection & & $12.06 \pm 0.25$ & $48.41 \pm 0.49$ & $45.13 \pm 0.48$ & $32.42 \pm 0.40$ & $5.87 \pm 0.17$ \\
\hline Trigger & 100 & $2.65 \pm 0.12$ & $6.97 \pm 0.19$ & $6.47 \pm 0.18$ & $4.87 \pm 0.16$ & $0.95 \pm 0.07$ \\
\hline Preselection & & $1.81 \pm 0.10$ & $4.94 \pm 0.16$ & $4.41 \pm 0.15$ & $2.59 \pm 0.11$ & $0.28 \pm 0.04$ \\
\hline Final selection & & $1.03 \pm 0.07$ & $3.47 \pm 0.13$ & $3.00 \pm 0.12$ & $1.64 \pm 0.09$ & $0.17 \pm 0.03$ \\
\hline
\end{tabular}


TABLE VI. Signal efficiencies for the model where the SM-like Higgs boson decays to two long-lived scalar particles $\mathrm{S}$ in the 2017 and 2018 analysis at different mean proper decay lengths $c \tau_{0}$ and with $m_{\mathrm{S}}=55 \mathrm{GeV}$. The long-lived scalar particle is assumed to decay to a down quark-antiquark pair $(S \rightarrow d \bar{d})$. Selection requirements are cumulative from the first row to the last. Uncertainties are statistical only.

\begin{tabular}{|c|c|c|c|c|c|c|}
\hline \multirow[b]{2}{*}{ Efficiency $\times 10^{4}$} & \multirow[b]{2}{*}{$m_{\mathrm{S}}(\mathrm{GeV})$} & \multicolumn{5}{|c|}{$\mathrm{c} \tau_{0}$} \\
\hline & & $1 \mathrm{~mm}$ & $10 \mathrm{~mm}$ & $30 \mathrm{~mm}$ & $100 \mathrm{~mm}$ & $1000 \mathrm{~mm}$ \\
\hline Trigger & 55 & $6.63 \pm 0.13$ & $32.07 \pm 0.29$ & $33.44 \pm 1.16$ & $25.25 \pm 0.26$ & $5.71 \pm 0.12$ \\
\hline Preselection & & $3.11 \pm 0.09$ & $13.61 \pm 0.19$ & $13.72 \pm 0.75$ & $9.39 \pm 0.16$ & $1.36 \pm 0.06$ \\
\hline Final selection & & $0.95 \pm 0.05$ & $6.12 \pm 0.13$ & $6.34 \pm 0.42$ & $4.46 \pm 0.11$ & $0.64 \pm 0.04$ \\
\hline
\end{tabular}

TABLE VII. Signal efficiencies for the model where the SM-like Higgs boson decays to two long-lived scalar particles S in the 2017 and 2018 analysis at different mean proper decay lengths $c \tau_{0}$ and with $m_{\mathrm{S}}=55 \mathrm{GeV}$. The long-lived scalar particle is assumed to decay to a bottom quark-antiquark pair $(\mathrm{S} \rightarrow b \bar{b})$. Selection requirements are cumulative from the first row to the last. Uncertainties are statistical only.

\begin{tabular}{|c|c|c|c|c|c|c|}
\hline \multirow[b]{2}{*}{ Efficiency $\times 10^{4}$} & \multirow[b]{2}{*}{$m_{\mathrm{S}}(\mathrm{GeV})$} & \multicolumn{5}{|c|}{$\mathrm{c} \tau_{0}$} \\
\hline & & $1 \mathrm{~mm}$ & $10 \mathrm{~mm}$ & $30 \mathrm{~mm}$ & $100 \mathrm{~mm}$ & $1000 \mathrm{~mm}$ \\
\hline Trigger & 55 & $4.30 \pm 0.11$ & $22.56 \pm 0.24$ & $24.45 \pm 0.48$ & $17.78 \pm 0.21$ & $4.05 \pm 0.10$ \\
\hline Preselection & & $0.66 \pm 0.04$ & $3.30 \pm 0.09$ & $3.97 \pm 0.19$ & $3.37 \pm 0.09$ & $0.57 \pm 0.04$ \\
\hline Final selection & & $0.08 \pm 0.01$ & $0.96 \pm 0.05$ & $1.17 \pm 0.10$ & $1.09 \pm 0.05$ & $0.19 \pm 0.02$ \\
\hline
\end{tabular}

TABLE VIII. Signal efficiencies for the $\tilde{g} \rightarrow g \tilde{G}$ model in the 2017 and 2018 analysis at different mean proper decay lengths $c \tau_{0}$ and different masses $m_{\tilde{g}}$. Selection requirements are cumulative from the first row to the last for each value of $m_{\tilde{g}}$. Uncertainties are statistical only.

\begin{tabular}{|c|c|c|c|c|c|c|}
\hline \multirow[b]{2}{*}{ Efficiency (\%) } & \multirow[b]{2}{*}{$m_{\tilde{g}}(\mathrm{GeV})$} & \multicolumn{5}{|c|}{$\mathrm{c} \tau_{0}$} \\
\hline & & $1 \mathrm{~mm}$ & $10 \mathrm{~mm}$ & $30 \mathrm{~mm}$ & $100 \mathrm{~mm}$ & $1000 \mathrm{~mm}$ \\
\hline Trigger & 2400 & $10.69 \pm 0.24$ & $69.70 \pm 0.60$ & $81.86 \pm 0.65$ & $82.86 \pm 0.66$ & $73.07 \pm 0.38$ \\
\hline Preselection & & $7.03 \pm 0.19$ & $63.62 \pm 0.57$ & $72.80 \pm 0.61$ & $69.67 \pm 0.60$ & $35.54 \pm 0.27$ \\
\hline Final selection & & $3.84 \pm 0.14$ & $44.20 \pm 0.48$ & $52.69 \pm 0.52$ & $50.84 \pm 0.52$ & $24.19 \pm 0.22$ \\
\hline Trigger & 1600 & $12.04 \pm 0.25$ & $68.36 \pm 0.59$ & $79.64 \pm 0.64$ & $79.96 \pm 0.64$ & $67.49 \pm 0.38$ \\
\hline Preselection & & $7.52 \pm 0.20$ & $60.55 \pm 0.56$ & $68.74 \pm 0.59$ & $63.47 \pm 0.57$ & $29.57 \pm 0.25$ \\
\hline Final selection & & $4.02 \pm 0.15$ & $41.33 \pm 0.46$ & $47.67 \pm 0.49$ & $43.16 \pm 0.47$ & $19.53 \pm 0.20$ \\
\hline Trigger & 1000 & $12.14 \pm 0.25$ & $62.77 \pm 0.56$ & $72.44 \pm 0.60$ & $71.90 \pm 0.60$ & $52.77 \pm 0.51$ \\
\hline Preselection & & $7.29 \pm 0.19$ & $53.19 \pm 0.52$ & $57.56 \pm 0.54$ & $52.25 \pm 0.51$ & $21.10 \pm 0.32$ \\
\hline Final selection & & $3.75 \pm 0.14$ & $34.57 \pm 0.42$ & $37.55 \pm 0.43$ & $33.84 \pm 0.41$ & $12.88 \pm 0.25$ \\
\hline
\end{tabular}

TABLE IX. Signal efficiencies for the $\tilde{g} \rightarrow q \bar{q} \tilde{\chi}_{1}^{0}$ model $\left(m_{\tilde{\chi}_{1}^{0}}=100 \mathrm{GeV}\right)$ in the 2017 and 2018 analysis at different mean proper decay lengths $c \tau_{0}$ and different masses $m_{\tilde{g}}$. Selection requirements are cumulative from the first row to the last for each value of $m_{\tilde{g}}$. Uncertainties are statistical only.

\begin{tabular}{|c|c|c|c|c|c|c|}
\hline \multirow[b]{2}{*}{ Efficiency (\%) } & \multirow[b]{2}{*}{$m_{\tilde{g}}(\mathrm{GeV})$} & \multicolumn{5}{|c|}{$\mathrm{c} \tau_{0}$} \\
\hline & & $1 \mathrm{~mm}$ & $10 \mathrm{~mm}$ & $30 \mathrm{~mm}$ & $100 \mathrm{~mm}$ & $1000 \mathrm{~mm}$ \\
\hline Trigger & 2600 & $15.08 \pm 0.28$ & $82.32 \pm 0.66$ & $90.46 \pm 0.69$ & $87.65 \pm 0.68$ & $78.78 \pm 0.65$ \\
\hline Preselection & & $9.91 \pm 0.23$ & $77.60 \pm 0.64$ & $85.82 \pm 0.67$ & $80.22 \pm 0.65$ & $43.24 \pm 0.48$ \\
\hline Final selection & & $5.75 \pm 0.17$ & $59.12 \pm 0.56$ & $68.44 \pm 0.60$ & $63.26 \pm 0.58$ & $30.84 \pm 0.41$ \\
\hline Trigger & 2000 & $17.70 \pm 0.31$ & $83.21 \pm 0.67$ & $90.46 \pm 0.69$ & $87.66 \pm 0.68$ & $79.04 \pm 0.65$ \\
\hline Preselection & & $11.20 \pm 0.24$ & $77.74 \pm 0.65$ & $85.04 \pm 0.67$ & $79.45 \pm 0.65$ & $39.69 \pm 0.46$ \\
\hline Final selection & & $6.57 \pm 0.19$ & $58.36 \pm 0.56$ & $66.29 \pm 0.59$ & $60.96 \pm 0.57$ & $27.12 \pm 0.38$ \\
\hline Trigger & 1600 & $19.39 \pm 0.32$ & $82.56 \pm 0.66$ & $90.27 \pm 0.69$ & $87.70 \pm 0.68$ & $78.31 \pm 0.64$ \\
\hline Preselection & & $12.16 \pm 0.26$ & $76.59 \pm 0.64$ & $84.13 \pm 0.59$ & $77.71 \pm 0.64$ & $37.41 \pm 0.44$ \\
\hline Final selection & & $6.76 \pm 0.19$ & $57.19 \pm 0.55$ & $64.37 \pm 0.58$ & $57.98 \pm 0.55$ & $25.02 \pm 0.36$ \\
\hline
\end{tabular}


TABLE X. Signal efficiencies for the $\tilde{g} \rightarrow t b s$ model in the 2017 and 2018 analysis at different mean proper decay lengths $c \tau_{0}$ and different masses $m_{\tilde{g}}$. Selection requirements are cumulative from the first row to the last for each value of $m_{\tilde{g}}$. Uncertainties are statistical only.

\begin{tabular}{|c|c|c|c|c|c|c|}
\hline \multirow[b]{2}{*}{ Efficiency (\%) } & \multirow[b]{2}{*}{$m_{\tilde{g}}(\mathrm{GeV})$} & \multicolumn{5}{|c|}{$\mathrm{c} \tau_{0}$} \\
\hline & & $1 \mathrm{~mm}$ & $10 \mathrm{~mm}$ & $30 \mathrm{~mm}$ & $100 \mathrm{~mm}$ & $1000 \mathrm{~mm}$ \\
\hline Trigger & 2600 & $25.14 \pm 0.39$ & $90.65 \pm 0.70$ & $95.78 \pm 0.73$ & $91.42 \pm 0.73$ & $83.61 \pm 0.67$ \\
\hline Preselection & & $16.26 \pm 0.31$ & $87.43 \pm 0.69$ & $94.08 \pm 0.73$ & $89.30 \pm 0.72$ & $56.49 \pm 0.55$ \\
\hline Final selection & & $9.60 \pm 0.24$ & $71.09 \pm 0.62$ & $81.12 \pm 0.67$ & $77.55 \pm 0.67$ & $42.62 \pm 0.48$ \\
\hline Trigger & 2000 & $29.89 \pm 0.42$ & $91.95 \pm 0.71$ & $95.53 \pm 0.73$ & $91.58 \pm 0.72$ & $83.38 \pm 0.68$ \\
\hline Preselection & & $18.93 \pm 0.34$ & $88.27 \pm 0.70$ & $93.55 \pm 0.72$ & $89.17 \pm 0.71$ & $52.54 \pm 0.54$ \\
\hline Final selection & & $11.29 \pm 0.26$ & $72.14 \pm 0.63$ & $79.58 \pm 0.67$ & $75.52 \pm 0.65$ & $38.12 \pm 0.46$ \\
\hline Trigger & 1600 & $31.61 \pm 0.43$ & $92.16 \pm 0.71$ & $95.58 \pm 0.76$ & $92.03 \pm 0.72$ & $84.50 \pm 0.67$ \\
\hline Preselection & & $20.06 \pm 0.34$ & $88.40 \pm 0.70$ & $93.46 \pm 0.75$ & $89.04 \pm 0.71$ & $50.46 \pm 0.52$ \\
\hline Final selection & & $11.71 \pm 0.26$ & $70.85 \pm 0.63$ & $78.52 \pm 0.69$ & $73.77 \pm 0.65$ & $35.91 \pm 0.44$ \\
\hline
\end{tabular}

TABLE XI. Signal efficiencies for the $\tilde{t} \rightarrow b \ell$ model in the 2017 and 2018 analysis at different mean proper decay lengths $c \tau_{0}$ and different masses $m_{\tilde{t}}$. Selection requirements are cumulative from the first row to the last for each value of $m_{\tilde{t}}$. Uncertainties are statistical only.

\begin{tabular}{|c|c|c|c|c|c|c|}
\hline \multirow[b]{2}{*}{ Efficiency (\%) } & \multirow[b]{2}{*}{$m_{\tilde{t}}(\mathrm{GeV})$} & \multicolumn{5}{|c|}{$\mathrm{c} \tau_{0}$} \\
\hline & & $1 \mathrm{~mm}$ & $10 \mathrm{~mm}$ & $30 \mathrm{~mm}$ & $100 \mathrm{~mm}$ & $1000 \mathrm{~mm}$ \\
\hline $\begin{array}{l}\text { Trigger } \\
\text { Preselection } \\
\text { Final selection }\end{array}$ & 1600 & $\begin{array}{r}18.00 \pm 0.30 \\
8.45 \pm 0.21 \\
5.03 \pm 0.16\end{array}$ & $\begin{array}{l}66.30 \pm 0.58 \\
54.41 \pm 0.53 \\
39.77 \pm 0.45\end{array}$ & $\begin{array}{l}74.36 \pm 0.62 \\
59.13 \pm 0.56 \\
42.85 \pm 0.48\end{array}$ & $\begin{array}{l}74.48 \pm 0.62 \\
52.63 \pm 0.52 \\
37.02 \pm 0.44\end{array}$ & $\begin{array}{l}66.67 \pm 0.59 \\
21.00 \pm 0.33 \\
12.58 \pm 0.25\end{array}$ \\
\hline $\begin{array}{l}\text { Trigger } \\
\text { Preselection } \\
\text { Final selection }\end{array}$ & 1000 & $\begin{array}{r}20.13 \pm 0.32 \\
8.32 \pm 0.21 \\
4.65 \pm 0.16\end{array}$ & $\begin{array}{l}65.37 \pm 0.58 \\
48.92 \pm 0.49 \\
33.46 \pm 0.41\end{array}$ & $\begin{array}{l}73.13 \pm 0.61 \\
53.45 \pm 0.53 \\
37.56 \pm 0.44\end{array}$ & $\begin{array}{l}73.74 \pm 0.61 \\
47.68 \pm 0.49 \\
31.81 \pm 0.40\end{array}$ & $\begin{array}{l}61.20 \pm 0.56 \\
16.99 \pm 0.30 \\
10.12 \pm 0.23\end{array}$ \\
\hline $\begin{array}{l}\text { Trigger } \\
\text { Preselection } \\
\text { Final selection }\end{array}$ & 600 & $\begin{array}{r}19.96 \pm 0.32 \\
6.85 \pm 0.19 \\
3.33 \pm 0.13\end{array}$ & $\begin{array}{l}58.90 \pm 0.54 \\
39.15 \pm 0.44 \\
24.44 \pm 0.35\end{array}$ & $\begin{array}{l}64.58 \pm 0.58 \\
42.25 \pm 0.47 \\
27.69 \pm 0.38\end{array}$ & $\begin{array}{l}64.93 \pm 0.58 \\
36.80 \pm 0.44 \\
23.12 \pm 0.35\end{array}$ & $\begin{array}{c}48.32 \pm 0.50 \\
11.59 \pm 0.25 \\
6.47 \pm 0.18\end{array}$ \\
\hline
\end{tabular}


TABLE XII. Signal efficiencies for the $\tilde{t} \rightarrow d \ell$ model in the 2017 and 2018 analysis at different mean proper decay lengths $c \tau_{0}$ and different masses $m_{\tilde{t}}$. Selection requirements are cumulative from the first row to the last for each value of $m_{\tilde{t}}$. Uncertainties are statistical only.

\begin{tabular}{|c|c|c|c|c|c|c|}
\hline \multirow[b]{2}{*}{ Efficiency (\%) } & \multirow[b]{2}{*}{$m_{\tilde{t}}(\mathrm{GeV})$} & \multicolumn{5}{|c|}{$\mathrm{c} \tau_{0}$} \\
\hline & & $1 \mathrm{~mm}$ & $10 \mathrm{~mm}$ & $30 \mathrm{~mm}$ & $100 \mathrm{~mm}$ & $1000 \mathrm{~mm}$ \\
\hline Trigger & 1600 & $18.58 \pm 0.31$ & $66.73 \pm 0.59$ & $74.69 \pm 0.63$ & $74.59 \pm 0.64$ & $67.27 \pm 0.60$ \\
\hline Preselection & & $10.72 \pm 0.24$ & $57.96 \pm 0.55$ & $63.08 \pm 0.58$ & $56.77 \pm 0.56$ & $22.79 \pm 0.35$ \\
\hline Final selection & & $6.62 \pm 0.19$ & $43.88 \pm 0.48$ & $47.92 \pm 0.51$ & $41.09 \pm 0.47$ & $14.33 \pm 0.28$ \\
\hline Trigger & 1000 & $19.95 \pm 0.33$ & $66.23 \pm 0.59$ & $73.87 \pm 0.63$ & $73.99 \pm 0.64$ & $62.66 \pm 0.57$ \\
\hline Preselection & & $9.71 \pm 0.23$ & $55.31 \pm 0.54$ & $59.93 \pm 0.57$ & $53.79 \pm 0.54$ & $19.80 \pm 0.32$ \\
\hline Final selection & & $5.60 \pm 0.17$ & $41.22 \pm 0.47$ & $44.38 \pm 0.49$ & $38.07 \pm 0.46$ & $12.34 \pm 0.25$ \\
\hline Trigger & 600 & $20.27 \pm 0.33$ & $60.49 \pm 0.58$ & $66.86 \pm 0.61$ & $66.85 \pm 0.61$ & $49.73 \pm 0.52$ \\
\hline Preselection & & $8.83 \pm 0.22$ & $47.48 \pm 0.51$ & $50.55 \pm 0.53$ & $44.37 \pm 0.50$ & $13.46 \pm 0.27$ \\
\hline Final selection & & $5.14 \pm 0.17$ & $33.96 \pm 0.43$ & $36.03 \pm 0.45$ & $30.16 \pm 0.41$ & $8.19 \pm 0.21$ \\
\hline
\end{tabular}

TABLE XIII. Signal efficiencies for the $\tilde{t} \rightarrow \bar{d} \bar{d}$ model in the 2017 and 2018 analysis at different mean proper decay lengths $c \tau_{0}$ and different masses $m_{\tilde{t}}$. Selection requirements are cumulative from the first row to the last for each value of $m_{\tilde{t}}$. Uncertainties are statistical only.

\begin{tabular}{|c|c|c|c|c|c|c|}
\hline \multirow[b]{2}{*}{ Efficiency $(\%)$} & \multirow[b]{2}{*}{$m_{\tilde{t}}(\mathrm{GeV})$} & \multicolumn{5}{|c|}{$\mathrm{c} \tau_{0}$} \\
\hline & & $1 \mathrm{~mm}$ & $10 \mathrm{~mm}$ & $30 \mathrm{~mm}$ & $100 \mathrm{~mm}$ & $1000 \mathrm{~mm}$ \\
\hline Trigger & 1600 & $22.10 \pm 0.35$ & $87.15 \pm 0.71$ & $92.19 \pm 0.72$ & $87.73 \pm 0.71$ & $79.12 \pm 0.67$ \\
\hline Preselection & & $17.06 \pm 0.31$ & $84.06 \pm 0.70$ & $88.49 \pm 0.71$ & $81.10 \pm 0.68$ & $39.89 \pm 0.48$ \\
\hline Final selection & & $11.78 \pm 0.26$ & $71.44 \pm 0.65$ & $76.02 \pm 0.66$ & $67.02 \pm 0.62$ & $28.52 \pm 0.40$ \\
\hline Trigger & 1000 & $23.86 \pm 0.36$ & $86.99 \pm 0.72$ & $91.58 \pm 0.77$ & $88.19 \pm 0.70$ & $78.31 \pm 0.66$ \\
\hline Preselection & & $16.96 \pm 0.30$ & $82.33 \pm 0.70$ & $86.73 \pm 0.75$ & $79.72 \pm 0.67$ & $35.91 \pm 0.45$ \\
\hline Final selection & & $11.91 \pm 0.26$ & $69.01 \pm 0.64$ & $73.52 \pm 0.69$ & $64.23 \pm 0.60$ & $25.52 \pm 0.38$ \\
\hline Trigger & 600 & $24.51 \pm 0.37$ & $84.75 \pm 0.68$ & $89.27 \pm 0.70$ & $85.55 \pm 0.68$ & $69.66 \pm 0.61$ \\
\hline Preselection & & $15.80 \pm 0.29$ & $78.05 \pm 0.66$ & $81.84 \pm 0.67$ & $74.51 \pm 0.64$ & $29.37 \pm 0.39$ \\
\hline Final selection & & $11.15 \pm 0.25$ & $64.32 \pm 0.60$ & $67.68 \pm 0.61$ & $58.85 \pm 0.57$ & $20.66 \pm 0.33$ \\
\hline
\end{tabular}



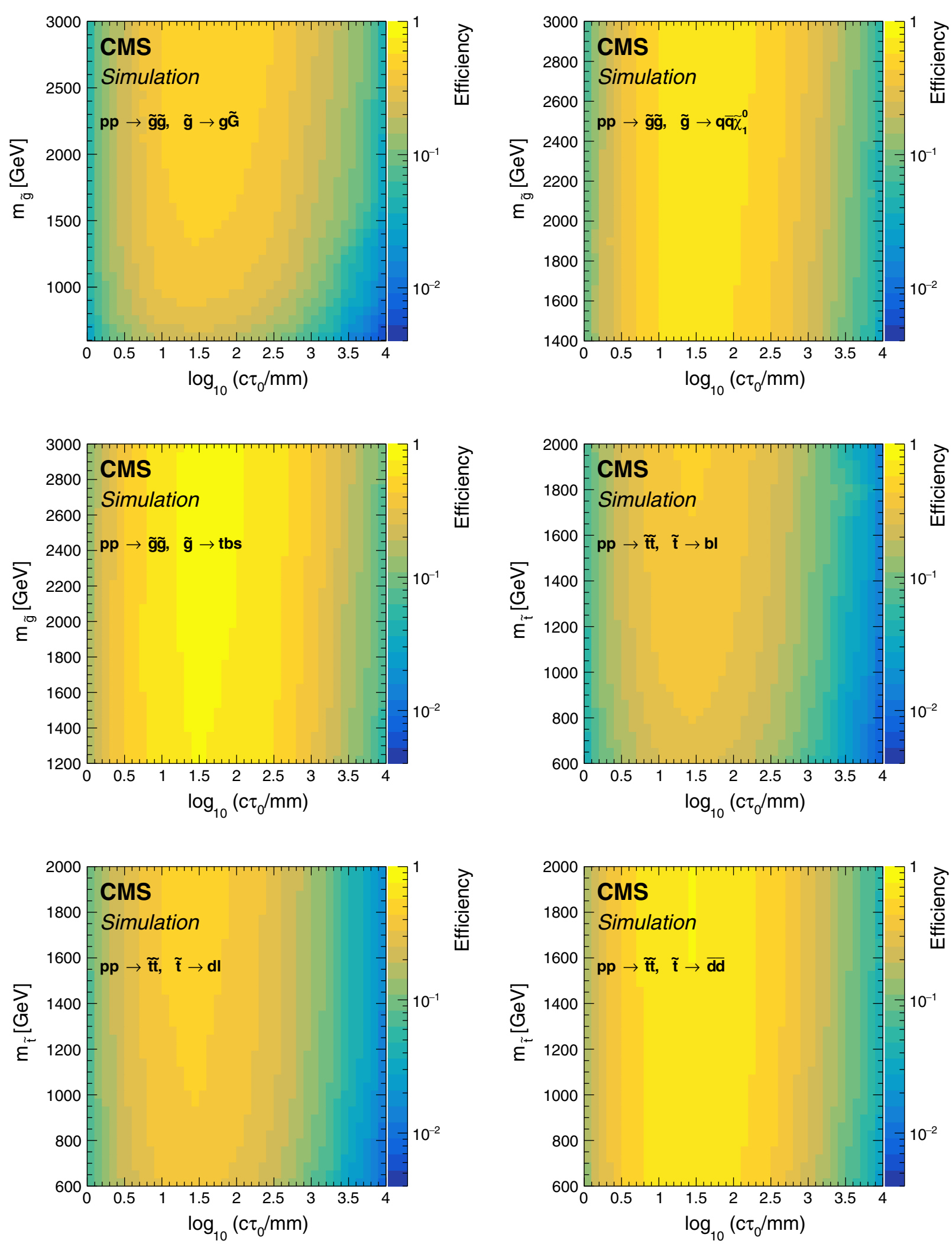

FIG. 15. The signal efficiencies as functions of the long-lived particle mass and mean proper decay length in the 2017 and 2018 analysis, for the $\tilde{g} \rightarrow g \tilde{G}$ model (upper left), the $\tilde{g} \rightarrow q \bar{q} \tilde{\chi}_{1}^{0}$ model (upper right), the $\tilde{g} \rightarrow t b s$ model (middle left), the $\tilde{t} \rightarrow b \ell$ model (middle right), the $\tilde{t} \rightarrow d \ell$ model (lower left), and the $\tilde{t} \rightarrow \bar{d} \bar{d}$ model (lower right). 
[1] N. Arkani-Hamed and S. Dimopoulos, Supersymmetric unification without low energy supersymmetry and signatures for fine-tuning at the LHC, J. High Energy Phys. 06 (2005) 073.

[2] G. F. Giudice and A. Romanino, Split supersymmetry, Nucl. Phys. B699, 65 (2004); Erratum, Nucl. Phys. B706, 487 (2005).

[3] J. L. Hewett, B. Lillie, M. Masip, and T. G. Rizzo, Signatures of long-lived gluinos in split supersymmetry, J. High Energy Phys. 09 (2004) 070.

[4] N. Arkani-Hamed, S. Dimopoulos, G. F. Giudice, and A. Romanino, Aspects of split supersymmetry, Nucl. Phys. B709, 3 (2005).

[5] P. Gambino, G. F. Giudice, and P. Slavich, Gluino decays in split supersymmetry, Nucl. Phys. B726, 35 (2005).

[6] A. Arvanitaki, N. Craig, S. Dimopoulos, and G. Villadoro, Mini-Split, J. High Energy Phys. 02 (2013) 126.

[7] N. Arkani-Hamed, A. Gupta, D. E. Kaplan, N. Weiner, and T. Zorawski, Simply unnatural supersymmetry, arXiv: 1212.6971.

[8] P. Fayet, Supergauge invariant extension of the Higgs mechanism and a model for the electron and its neutrino, Nucl. Phys. B90, 104 (1975).

[9] G. R. Farrar and P. Fayet, Phenomenology of the production, decay, and detection of new hadronic states associated with supersymmetry, Phys. Lett. 76B, 575 (1978).

[10] S. Weinberg, Supersymmetry at ordinary energies. 1. Masses and conservation laws, Phys. Rev. D 26, 287 (1982).

[11] L. J. Hall and M. Suzuki, Explicit R-parity breaking in supersymmetric models, Nucl. Phys. B231, 419 (1984).

[12] R. Barbier, C. Bérat, M. Besançon, M. Chemtob, A. Deandrea, E. Dudas, P. Fayet, S. Lavignac, G. Moreau, E. Perez, and Y. Sirois, $R$-parity violating supersymmetry, Phys. Rep. 420, 1 (2005).

[13] G. F. Giudice and R. Rattazzi, Theories with gauge mediated supersymmetry breaking, Phys. Rep. 322, 419 (1999).

[14] P. Meade, N. Seiberg, and D. Shih, General gauge mediation, Prog. Theor. Phys. Suppl. 177, 143 (2009).

[15] M. Buican, P. Meade, N. Seiberg, and D. Shih, Exploring general gauge mediation, J. High Energy Phys. 03 (2009) 016.

[16] J. Fan, M. Reece, and J. T. Ruderman, Stealth supersymmetry, J. High Energy Phys. 11 (2011) 012.

[17] J. Fan, M. Reece, and J. T. Ruderman, A stealth supersymmetry sampler, J. High Energy Phys. 07 (2012) 196.

[18] M. J. Strassler and K. M. Zurek, Echoes of a hidden valley at hadron colliders, Phys. Lett. B 651, 374 (2007).

[19] M. J. Strassler and K. M. Zurek, Discovering the Higgs through highly-displaced vertices, Phys. Lett. B 661, 263 (2008).

[20] T. Han, Z. Si, K. M. Zurek, and M. J. Strassler, Phenomenology of hidden valleys at hadron colliders, J. High Energy Phys. 07 (2008) 008.

[21] D. E. Kaplan, M. A. Luty, and K. M. Zurek, Asymmetric dark matter, Phys. Rev. D 79, 115016 (2009).

[22] L. J. Hall, K. Jedamzik, J. March-Russell, and S. M. West, Freeze-in production of FIMP dark matter, J. High Energy Phys. 03 (2010) 080.
[23] I.-W. Kim and K. M. Zurek, Flavor and collider signatures of asymmetric dark matter, Phys. Rev. D 89, 035008 (2014).

[24] Y. Cui and B. Shuve, Probing baryogenesis with displaced vertices at the LHC, J. High Energy Phys. 02 (2015) 049.

[25] R. T. Co, F. D’Eramo, L. J. Hall, and D. Pappadopulo, Freeze-In dark matter with displaced signatures at colliders, J. Cosmol. Astropart. Phys. 12 (2015) 024.

[26] L. Calibbi, L. Lopez-Honorez, S. Lowette, and A. Mariotti, Singlet-doublet dark matter freeze-in: LHC displaced signatures versus cosmology, J. High Energy Phys. 09 (2018) 037.

[27] Y. Cui, L. Randall, and B. Shuve, A WIMPy baryogenesis miracle, J. High Energy Phys. 04 (2012) 075.

[28] Y. Cui and R. Sundrum, Baryogenesis for weakly interacting massive particles, Phys. Rev. D 87, 116013 (2013).

[29] A. Atre, T. Han, S. Pascoli, and B. Zhang, The search for heavy Majorana neutrinos, J. High Energy Phys. 05 (2009) 030 .

[30] M. Drewes, The phenomenology of right handed neutrinos, Int. J. Mod. Phys. E 22, 1330019 (2013).

[31] F. F. Deppisch, P.S. Bhupal Dev, and A. Pilaftsis, Neutrinos and collider physics, New J. Phys. 17, 075019 (2015).

[32] Y. Cai, T. Han, T. Li, and R. Ruiz, Lepton number violation: Seesaw models and their collider tests, Front. Phys. 6, 40 (2018).

[33] Z. Chacko, H.-S. Goh, and R. Harnik, Natural Electroweak Breaking from a Mirror Symmetry, Phys. Rev. Lett. 96, 231802 (2006).

[34] H. Cai, H.-C. Cheng, and J. Terning, A quirky little Higgs model, J. High Energy Phys. 05 (2009) 045.

[35] N. Craig, S. Knapen, and P. Longhi, Neutral Naturalness from Orbifold Higgs Models, Phys. Rev. Lett. 114, 061803 (2015).

[36] N. Craig, A. Katz, M. Strassler, and R. Sundrum, Naturalness in the dark at the LHC, J. High Energy Phys. 07 (2015) 105.

[37] D. Curtin and C. B. Verhaaren, Discovering uncolored naturalness in exotic Higgs decays, J. High Energy Phys. 12 (2015) 072.

[38] S. Alipour-Fard, N. Craig, S. Gori, S. Koren, and D. Redigolo, The second Higgs at the lifetime frontier, J. High Energy Phys. 07 (2020) 029.

[39] CMS Collaboration, The CMS experiment at the CERN LHC, J. Instrum. 3, S08004 (2008).

[40] CMS Collaboration, Search for long-lived particles decaying into displaced jets in proton-proton collisions at $\sqrt{s}=13 \mathrm{TeV}$, Phys. Rev. D 99, 032011 (2019).

[41] ATLAS Collaboration, Search for long-lived, massive particles in events with displaced vertices and missing transverse momentum in $\sqrt{s}=13 \mathrm{TeV} p p$ collisions with the ATLAS detector, Phys. Rev. D 97, 052012 (2018).

[42] ATLAS Collaboration, Search for long-lived particles produced in $p p$ collisions at $\sqrt{s}=13 \mathrm{TeV}$ that decay into displaced hadronic jets in the ATLAS muon spectrometer, Phys. Rev. D 99, 052005 (2019).

[43] ATLAS Collaboration, Search for long-lived neutral particles in $p p$ collisions at $\sqrt{s}=13 \mathrm{TeV}$ that decay into 
displaced hadronic jets in the ATLAS calorimeter, Eur. Phys. J. C 79, 481 (2019).

[44] ATLAS Collaboration, Search for long-lived, massive particles in events with a displaced vertex and a muon with large impact parameter in $p p$ collisions at $\sqrt{s}=$ $13 \mathrm{TeV}$ with the ATLAS detector, Phys. Rev. D 102, 032006 (2020).

[45] ATLAS Collaboration, Search for long-lived neutral particles produced in $p p$ collisions at $\sqrt{s}=13 \mathrm{TeV}$ decaying into displaced hadronic jets in the ATLAS inner detector and muon spectrometer, Phys. Rev. D 101, 052013 (2020).

[46] CMS Collaboration, Search for new long-lived particles at $\sqrt{s}=13 \mathrm{TeV}$, Phys. Lett. B 780, 432 (2018).

[47] CMS Collaboration, Search for long-lived particles with displaced vertices in multijet events in proton-proton collisions at $\sqrt{s}=13 \mathrm{TeV}$, Phys. Rev. D 98, 092011 (2018).

[48] CMS Collaboration, Search for long-lived particles using nonprompt jets and missing transverse momentum with proton-proton collisions at $\sqrt{s}=13 \mathrm{TeV}$, Phys. Lett. B 797, 134876 (2019).

[49] CMS Collaboration, Description and performance of track and primary-vertex reconstruction with the CMS tracker, J. Instrum. 9, P10009 (2014).

[50] CMS Collaboration, The CMS trigger system, J. Instrum. 12, P01020 (2017).

[51] M. Cacciari, G. P. Salam, and G. Soyez, The anti- $k_{\mathrm{T}}$ jet clustering algorithm, J. High Energy Phys. 04 (2008) 063.

[52] M. Cacciari, G. P. Salam, and G. Soyez, FastJet user manual, Eur. Phys. J. C 72, 1896 (2012).

[53] CMS Collaboration, Jet energy scale and resolution in the CMS experiment in pp collisions at $8 \mathrm{TeV}$, J. Instrum. 12, P02014 (2017).

[54] CMS Collaboration, Technical proposal for the Phase-II upgrade of the CMS detector, CMS Technical proposal CERN-LHCC-2015-010, CMS-TDR-15-02, 2015.

[55] J. Alwall, R. Frederix, S. Frixione, V. Hirschi, F. Maltoni, O. Mattelaer, H. S. Shao, T. Stelzer, P. Torrielli, and M. Zaro, The automated computation of tree-level and nextto-leading order differential cross sections, and their matching to parton shower simulations, J. High Energy Phys. 07 (2014) 079.

[56] T. Sjöstrand, S. Ask, J. R. Christiansen, R. Corke, N. Desai, P. Ilten, S. Mrenna, S. Prestel, C. O. Rasmussen, and P. Z. Skands, An introduction to PYTHIA8.2, Comput. Phys. Commun. 191, 159 (2015).

[57] J. Alwall et al., Comparative study of various algorithms for the merging of parton showers and matrix elements in hadronic collisions, Eur. Phys. J. C 53, 473 (2008).

[58] CMS Collaboration, Extraction and validation of a new set of CMS PYTHIA8 tunes from underlying-event measurements, Eur. Phys. J. C 80, 4 (2020).

[59] R. D. Ball et al. (NNPDF Collaboration), Parton distributions from high-precision collider data, Eur. Phys. J. C 77, 663 (2017).

[60] LHC Higgs Cross Section Working Group, Handbook of LHC Higgs cross sections: 4. Deciphering the nature of the Higgs sector, CERN Report CERN-2017-002-M, 2016.
[61] G. Burdman, Z. Chacko, H.-S. Goh, and R. Harnik, Folded supersymmetry and the LEP paradox, J. High Energy Phys. 02 (2007) 009.

[62] P. Nason, A new method for combining NLO QCD with shower Monte Carlo algorithms, J. High Energy Phys. 11 (2004) 040.

[63] S. Frixione, P. Nason, and C. Oleari, Matching NLO QCD computations with parton shower simulations: The POWHEG method, J. High Energy Phys. 11 (2007) 070.

[64] S. Alioli, P. Nason, C. Oleari, and E. Re, A general framework for implementing NLO calculations in shower Monte Carlo programs: The POWHEG BOX, J. High Energy Phys. 06 (2010) 043.

[65] E. Bagnaschi, G. Degrassi, P. Slavich, and A. Vicini, Higgs production via gluon fusion in the POWHEG approach in the SM and in the MSSM, J. High Energy Phys. 02 (2012) 088 .

[66] Z. Liu and B. Tweedie, The fate of long-lived superparticles with hadronic decays after LHC Run 1, J. High Energy Phys. 06 (2015) 042.

[67] C. Csaki, Y. Grossman, and B. Heidenreich, Minimal flavor violation supersymmetry: A natural theory for $R$ parity violation, Phys. Rev. D 85, 095009 (2012).

[68] P. W. Graham, D. E. Kaplan, S. Rajendran, and P. Saraswat, Displaced supersymmetry, J. High Energy Phys. 07 (2012) 149.

[69] C. Csaki, E. Kuflik, and T. Volansky, Dynamical R-Parity Violation, Phys. Rev. Lett. 112, 131801 (2014).

[70] C. Csaki, E. Kuflik, O. Slone, and T. Volansky, Models of dynamical $R$-parity violation, J. High Energy Phys. 06 (2015) 045.

[71] C. Csaki, E. Kuflik, S. Lombardo, O. Slone, and T. Volansky, Phenomenology of a long-lived LSP with $R$-parity violation, J. High Energy Phys. 08 (2015) 016.

[72] G. R. Farrar and P. Fayet, Bounds on $R$-hadron production from calorimetry experiments, Phys. Lett. 79B, 442 (1978).

[73] M. Fairbairn, A. C. Kraan, D. A. Milstead, T. Sjostrand, P.Z. Skands, and T. Sloan, Stable massive particles at colliders, Phys. Rep. 438, 1 (2007).

[74] R. Mackeprang and A. Rizzi, Interactions of coloured heavy stable particles in matter, Eur. Phys. J. C 50, 353 (2007).

[75] R. Mackeprang and D. Milstead, An updated description of heavy-hadron interactions in GEANT-4, Eur. Phys. J. C 66, 493 (2010).

[76] S. Agostinelli et al. (GEANT4 Collaboration), GEANT4A simulation toolkit, Nucl. Instrum. Methods Phys. Res., Sect. A 506, 250 (2003).

[77] R. Frühwirth, W. Waltenberger, and P. Vanlaer, Adaptive vertex fitting, J. Phys. G 34, N343 (2007).

[78] CMS Collaboration, Precision measurement of the structure of the CMS inner tracking system using nuclear interactions, J. Instrum. 13, P10034 (2018).

[79] CMS Collaboration, CMS technical design report for the pixel detector upgrade, Technical Report No. CERNLHCC-2012-016, CMS-TDR-011, 2012.

[80] S. C. Johnson, Hierarchical clustering schemes, Psychometrika 32, 241 (1967). 
[81] Y. Freund and R. E. Schapire, Experiments with a new boosting algorithm, in Proceedings of the Thirteenth International Conference on International Conference on Machine Learning, ICML'96 (Morgan Kaufmann Publishers Inc., San Francisco, CA, USA, 1996), p. 148.

[82] J. Friedman, T. Hastie, and R. Tibshirani, Additive logistic regression: A statistical view of boosting (with discussion and a rejoinder by the authors), Ann. Stat. 28, 337 (2000).

[83] J.H. Friedman, Greedy function approximation: A gradient boosting machine, Ann. Stat. 29, 1189 (2001).

[84] H. Voss, A. Höcker, J. Stelzer, and F. Tegenfeldt, TMVA, the toolkit for multivariate data analysis with ROOT, in XIth International Workshop on Advanced Computing and Analysis Techniques in Physics Research (ACAT) (2007), p. 40.

[85] F. Pedregosa et al., Scikit-learn: Machine learning in PYTHON, J. Mach. Learn. Res. 12, 2825 (2011), https:// www.jmlr.org/papers/volume12/pedregosa11a/ pedregosa11a.pdf.

[86] G. Punzi, Sensitivity of searches for new signals and its optimization, in Statistical Problems in Particle Physics, Astrophysics and Cosmology. Proceedings, Conference, PHYSTAT 2003, Stanford, USA, 2003 (2003), p. MODT002, [eConf C030908/MOTD002], arXiv:physics/0308063.

[87] CMS Collaboration, CMS luminosity measurement for the 2017 data-taking period at $\sqrt{s}=13 \mathrm{TeV}$, CMS Physics Analysis Summary CMS-PAS-LUM-17-004, 2018.

[88] CMS Collaboration, CMS luminosity measurement for the 2018 data-taking period at $\sqrt{s}=13 \mathrm{TeV}, \mathrm{CMS}$ Physics Analysis Summary CMS-PAS-LUM-18-002, 2019.

[89] CMS Collaboration, Measurement of the inelastic protonproton cross section at $\sqrt{s}=13 \mathrm{TeV}$, J. High Energy Phys. 07 (2018) 161.

[90] S. Dulat, T.-J. Hou, J. Gao, M. Guzzi, J. Huston, P. Nadolsky, J. Pumplin, C. Schmidt, D. Stump, and C. P. Yuan, New parton distribution functions from a global analysis of quantum chromodynamics, Phys. Rev. D 93, 033006 (2016).

[91] L. A. Harland-Lang, A. D. Martin, P. Motylinski, and R. S. Thorne, Parton distributions in the LHC era: MMHT 2014 PDFs, Eur. Phys. J. C 75, 204 (2015).
[92] J. Butterworth et al., PDF4LHC recommendations for LHC Run II, J. Phys. G 43, 023001 (2016).

[93] A. Buckley, J. Ferrando, S. Lloyd, K. Nordström, B. Page, M. Rüfenacht, M. Schönherr, and G. Watt, LHAPDF6: Parton density access in the LHC precision era, Eur. Phys. J. C 75, 132 (2015).

[94] T. Junk, Confidence level computation for combining searches with small statistics, Nucl. Instrum. Methods Phys. Res., Sect. A 434, 435 (1999).

[95] A.L. Read, Presentation of search results: The $\mathrm{CL}_{\mathrm{s}}$ technique, J. Phys. G 28, 2693 (2002).

[96] G. Cowan, K. Cranmer, E. Gross, and O. Vitells, Asymptotic formulae for likelihood-based tests of new physics, Eur. Phys. J. C 71, 1554 (2011).

[97] ATLAS and CMS Collaborations, The LHC Higgs Combination group, Procedure for the LHC Higgs boson search combination in Summer 2011, Technical Report No. CMS-NOTE-2011-005, ATL-PHYS-PUB-2011-11, 2011.

[98] W. Beenakker, R. Hopker, M. Spira, and P. M. Zerwas, Squark and gluino production at hadron colliders, Nucl. Phys. B492, 51 (1997).

[99] A. Kulesza and L. Motyka, Threshold Resummation for Squark-Antisquark and Gluino-Pair Production at the LHC, Phys. Rev. Lett. 102, 111802 (2009).

[100] A. Kulesza and L. Motyka, Soft gluon resummation for the production of gluino-gluino and squark-antisquark pairs at the LHC, Phys. Rev. D 80, 095004 (2009).

[101] W. Beenakker, S. Brensing, M. n. Kramer, A. Kulesza, E. Laenen, L. Motyka, and I. Niessen, Squark and gluino hadroproduction, Int. J. Mod. Phys. A 26, 2637 (2011).

[102] C. Borschensky, M. Krämer, A. Kulesza, M. Mangano, S. Padhi, T. Plehn, and X. Portell, Squark and gluino production cross sections in pp collisions at $\sqrt{s}=13$, 14, 33 and 100 TeV, Eur. Phys. J. C 74, 3174 (2014).

[103] W. Beenakker, C. Borschensky, M. Krämer, A. Kulesza, and E. Laenen, NNLL-fast: Predictions for coloured supersymmetric particle production at the LHC with threshold and Coulomb resummation, J. High Energy Phys. 12 (2016) 133.

A. M. Sirunyan, ${ }^{1, a}$ A. Tumasyan, ${ }^{1}$ W. Adam, ${ }^{2}$ F. Ambrogi, ${ }^{2}$ T. Bergauer, ${ }^{2}$ M. Dragicevic, ${ }^{2}$ J. Erö, ${ }^{2}$ A. Escalante Del Valle, ${ }^{2}$ R. Frühwirth, ${ }^{2, b}$ M. Jeitler, ${ }^{2, b}$ N. Krammer, ${ }^{2}$ L. Lechner, ${ }^{2}$ D. Liko, ${ }^{2}$ T. Madlener, ${ }^{2}$ I. Mikulec, ${ }^{2}$ F. M. Pitters, ${ }^{2}$ N. Rad, ${ }^{2}$ J. Schieck, ${ }^{2, b}$ R. Schöfbeck, ${ }^{2}$ M. Spanring, ${ }^{2}$ S. Templ, ${ }^{2}$ W. Waltenberger, ${ }^{2}$ C.-E. Wulz, ${ }^{2, b}$ M. Zarucki, ${ }^{2}$ V. Chekhovsky, ${ }^{3}$ A. Litomin, ${ }^{3}$ V. Makarenko, ${ }^{3}$ J. Suarez Gonzalez, ${ }^{3}$ M. R. Darwish,${ }^{4, \mathrm{c}}$ E. A. De Wolf, ${ }^{4}$ D. Di Croce, ${ }^{4}$ X. Janssen, ${ }^{4}$ T. Kello, ${ }^{4, d}$ A. Lelek, ${ }^{4}$ M. Pieters, ${ }^{4}$ H. Rejeb Sfar ${ }^{4}$ H. Van Haevermaet, ${ }^{4}$ P. Van Mechelen, ${ }^{4}$ S. Van Putte, ${ }^{4}$ N. Van Remortel, ${ }^{4}$ F. Blekman, ${ }^{5}$ E. S. Bols, ${ }^{5}$ S. S. Chhibra, ${ }_{5}^{5}$ J. D'Hondt ${ }^{5}$ J. De Clercq, ${ }^{5}$ D. Lontkovskyi, ${ }^{5}$ S. Lowette, ${ }^{5}$ I. Marchesini, S. Moortgat, ${ }^{5}$ A. Morton, ${ }^{5}$ Q. Python, ${ }^{5}$ S. Tavernier, ${ }^{5}$ W. Van Doninck, ${ }^{5}$ P. Van Mulders, ${ }^{5}$ D. Beghin, ${ }^{6}$ B. Bilin, ${ }^{6}$ B. Clerbaux, ${ }^{6}$ G. De Lentdecker, ${ }^{6}$ B. Dorney, ${ }^{6}$ L. Favart, ${ }^{6}$ A. Grebenyuk, ${ }^{6}$ A. K. Kalsi, ${ }^{6}$ I. Makarenko, ${ }^{6}$ L. Moureaux, ${ }^{6}$ L. Pétré, ${ }^{6}$ A. Popov, ${ }^{6}$ N. Postiau, ${ }^{6}$ E. Starling, ${ }^{6}$ L. Thomas, ${ }^{6}$ C. Vander Velde, ${ }^{6}$ P. Vanlaer, ${ }^{6}$ D. Vannerom, ${ }^{6}$ L. Wezenbeek, ${ }^{6}$ T. Cornelis, ${ }^{7}$ D. Dobur, ${ }^{7}$ M. Gruchala, ${ }^{7}$ I. Khvastunov, ${ }^{7, \mathrm{e}}$ M. Niedziela, ${ }^{7}$ C. Roskas, ${ }^{7}$ K. Skovpen, ${ }^{7}$ M. Tytgat, ${ }^{7}$ W. Verbeke, ${ }^{7}$ B. Vermassen, ${ }^{7}$ M. Vit, ${ }^{7}$ G. Bruno, ${ }^{8}$ F. Bury,${ }^{8}$ C. Caputo, ${ }^{8}$ P. David,${ }^{8}$ C. Delaere, ${ }^{8}$ M. Delcourt, ${ }^{8}$ I. S. Donertas, ${ }^{8}$ 
A. Giammanco, ${ }^{8}$ V. Lemaitre, ${ }^{8}$ K. Mondal,${ }^{8}$ J. Prisciandaro, ${ }^{8}$ A. Taliercio, ${ }^{8}$ M. Teklishyn, ${ }^{8}$ P. Vischia, ${ }^{8}$ S. Wuyckens, ${ }^{8}$ J. Zobec, ${ }^{8}$ G. A. Alves, ${ }^{9}$ G. Correia Silva, ${ }^{9}$ C. Hensel,${ }^{9}$ A. Moraes, ${ }^{9}$ W. L. Aldá Júnior, ${ }^{10}$ E. Belchior Batista Das Chagas, ${ }^{10}$ H. Brandao Malbouisson, ${ }^{10}$ W. Carvalho, ${ }^{10}$ J. Chinellato, ${ }^{10, \mathrm{f}}$ E. Coelho, ${ }^{10}$ E. M. Da Costa, ${ }^{10}$ G. G. Da Silveira, ${ }^{10, g}$ D. De Jesus Damiao, ${ }^{10}$ S. Fonseca De Souza,${ }^{10}$ J. Martins,${ }^{10, h}$ D. Matos Figueiredo, ${ }^{10}$ M. Medina Jaime, ${ }^{10, i}$ M. Melo De Almeida, ${ }^{10}$ C. Mora Herrera, ${ }^{10}$ L. Mundim, ${ }^{10}$ H. Nogima, ${ }^{10}$ P. Rebello Teles, ${ }^{10}$ L. J. Sanchez Rosas, ${ }^{10}$ A. Santoro, ${ }^{10}$ S. M. Silva Do Amaral, ${ }^{10}$ A. Sznajder, ${ }^{10}$ M. Thiel, ${ }^{10}$ E. J. Tonelli Manganote,${ }^{10, f}$

F. Torres Da Silva De Araujo, ${ }^{10}$ A. Vilela Pereira, ${ }^{10}$ C. A. Bernardes, ${ }^{11 a}$ L. Calligaris, ${ }^{11 a}$ T. R. Fernandez Perez Tomei, ${ }^{11 a}$ E. M. Gregores, ${ }^{11 a, 11 b}$ D. S. Lemos, ${ }^{11 a}$ P. G. Mercadante, ${ }^{11 a, 11 b}$ S. F. Novaes, ${ }^{11 a}$ Sandra S. Padula, ${ }^{11 a}$ A. Aleksandrov, ${ }^{12}$ G. Antchev, ${ }^{12}$ I. Atanasov, ${ }^{12}$ R. Hadjiiska, ${ }^{12}$ P. Iaydjiev, ${ }^{12}$ M. Misheva, ${ }^{12}$ M. Rodozov, ${ }^{12}$ M. Shopova, ${ }^{12}$ G. Sultanov, ${ }^{12}$ M. Bonchev, ${ }^{13}$ A. Dimitrov, ${ }^{13}$ T. Ivanov, ${ }^{13}$ L. Litov, ${ }^{13}$ B. Pavlov, ${ }^{13}$ P. Petkov, ${ }^{13}$ A. Petrov, ${ }^{13}$ W. Fang, ${ }^{14, d}$ Q. Guo, ${ }^{14}$ H. Wang, ${ }^{14}$ L. Yuan, ${ }^{14}$ M. Ahmad, ${ }^{15}$ Z. Hu, ${ }^{15}$ Y. Wang, ${ }^{15}$ E. Chapon, ${ }^{16}$ G. M. Chen, ${ }^{16, j}$ H. S. Chen, ${ }^{16, j}$ M. Chen, ${ }^{16}$ D. Leggat, ${ }^{16}$ H. Liao, ${ }^{16}$ Z. Liu, ${ }^{16}$ R. Sharma,${ }^{16}$ A. Spiezia, ${ }^{16}$ J. Tao, ${ }^{16}$ J. Thomas-Wilsker, ${ }^{16}$ J. Wang, ${ }^{16}$ H. Zhang, ${ }^{16}$ S. Zhang, ${ }^{16, j}$ J. Zhao, ${ }^{16}$ A. Agapitos,${ }^{17}$ Y. Ban,${ }^{17}$ C. Chen, ${ }^{17}$ A. Levin, ${ }^{17}$ Q. Li,${ }^{17}$ M. Lu, ${ }^{17}$ X. Lyu,${ }^{17}$ Y. Mao, ${ }^{17}$ S. J. Qian, ${ }^{17}$ D. Wang, ${ }^{17}$ Q. Wang, ${ }^{17}$ J. Xiao, ${ }^{17}$ Z. You, ${ }^{18}$ X. Gao, ${ }^{19, d}$ M. Xiao, ${ }^{20}$ C. Avila, ${ }^{21}$ A. Cabrera,${ }^{21}$ C. Florez, ${ }^{21}$ J. Fraga, ${ }^{21}$ A. Sarkar, ${ }^{21}$ M. A. Segura Delgado, ${ }^{21}$ J. Jaramillo, ${ }^{22}$ J. Mejia Guisao, ${ }^{22}$ F. Ramirez, ${ }^{22}$ J. D. Ruiz Alvarez, ${ }^{22}$ C. A. Salazar González, ${ }^{22}$ N. Vanegas Arbelaez, ${ }^{22}$ D. Giljanovic, ${ }^{23}$ N. Godinovic, ${ }^{23}$ D. Lelas, ${ }^{23}$ I. Puljak, ${ }^{23}$ T. Sculac, ${ }^{23}$ Z. Antunovic, ${ }^{24}$ M. Kovac, ${ }^{24}$ V. Brigljevic, ${ }^{25}$ D. Ferencek ${ }^{25}$ D. Majumder ${ }^{25}$ M. Roguljic, ${ }^{25}$ A. Starodumov, ${ }^{25, k}$ T. Susa, ${ }^{25}$ M. W. Ather, ${ }^{26}$ A. Attikis, ${ }^{26}$ E. Erodotou, ${ }^{26}$ A. Ioannou, ${ }^{26}$ G. Kole, ${ }^{26}$ M. Kolosova, ${ }^{26}$ S. Konstantinou, ${ }^{26}$

G. Mavromanolakis, ${ }^{26}$ J. Mousa, ${ }^{26}$ C. Nicolaou, ${ }^{26}$ F. Ptochos, ${ }^{26}$ P. A. Razis,${ }^{26}$ H. Rykaczewski, ${ }^{26}$ H. Saka, ${ }^{26}$ D. Tsiakkouri, ${ }^{26}$ M. Finger ${ }^{27,1}$ M. Finger Jr., ${ }^{27,1}$ A. Kveton, ${ }^{27}$ J. Tomsa, ${ }^{27}$ E. Ayala, ${ }^{28}$ E. Carrera Jarrin, ${ }^{29}$ S. Abu Zeid, ${ }^{30, m}$ S. Elgammal, ${ }^{30, n}$ A. Ellithi Kamel, ${ }^{30,0}$ M. A. Mahmoud, ${ }^{31}$ Y. Mohammed, ${ }^{31, p}$ S. Bhowmik, ${ }^{32}$ A. Carvalho Antunes De Oliveira, ${ }^{32}$ R. K. Dewanjee, ${ }^{32}$ K. Ehataht,${ }^{32}$ M. Kadastik, ${ }^{32}$ M. Raidal, ${ }^{32}$ C. Veelken, ${ }^{32}$ P. Eerola, ${ }^{33}$ L. Forthomme, ${ }^{33}$ H. Kirschenmann, ${ }^{33}$ K. Osterberg, ${ }^{33}$ M. Voutilainen, ${ }^{33}$ E. Brücken, ${ }^{34}$ F. Garcia ${ }^{34}$ J. Havukainen, ${ }^{34}$ V. Karimäki, ${ }^{34}$ M. S. Kim, ${ }^{34}$ R. Kinnunen,${ }^{34}$ T. Lampén, ${ }^{34}$ K. Lassila-Perini, ${ }^{34}$ S. Laurila, ${ }^{34}$ S. Lehti, ${ }^{34}$ T. Lindén, ${ }^{34}$ H. Siikonen, ${ }^{34}$ E. Tuominen, ${ }^{34}$ J. Tuominiemi, ${ }^{34}$ P. Luukka, ${ }^{35}$ T. Tuuva, ${ }^{35}$ C. Amendola,${ }^{36}$ M. Besancon, ${ }^{36}$ F. Couderc, ${ }^{36}$ M. Dejardin, ${ }^{36}$ D. Denegri, ${ }^{36}$ J. L. Faure, ${ }^{36}$ F. Ferri ${ }^{36}$ S. Ganjour, ${ }^{36}$ A. Givernaud ${ }^{36}$ P. Gras, ${ }^{36}$ G. Hamel de Monchenault, ${ }^{36}$ P. Jarry, ${ }^{36}$ B. Lenzi, ${ }^{36}$ E. Locci, ${ }^{36}$ J. Malcles, ${ }^{36}$ J. Rander, ${ }^{36}$ A. Rosowsky, ${ }^{36}$ M. Ö. Sahin, ${ }^{36}$ A. Savoy-Navarro, ${ }^{36, q}$ M. Titov, ${ }^{36}$ G. B. Yu, ${ }^{36}$ S. Ahuja ${ }^{37}$ F. Beaudette, ${ }^{37}$

M. Bonanomi, ${ }^{37}$ A. Buchot Perraguin, ${ }^{37}$ P. Busson, ${ }^{37}$ C. Charlot,${ }^{37}$ O. Davignon, ${ }^{37}$ B. Diab, ${ }^{37}$ G. Falmagne, ${ }^{37}$

R. Granier de Cassagnac, ${ }^{37}$ A. Hakimi ${ }^{37}$ I. Kucher, ${ }^{37}$ A. Lobanov, ${ }^{37}$ C. Martin Perez,${ }^{37}$ M. Nguyen, ${ }^{37}$ C. Ochando, ${ }^{37}$ P. Paganini, ${ }^{37}$ J. Rembser ${ }^{37}$ R. Salerno,${ }^{37}$ J. B. Sauvan, ${ }^{37}$ Y. Sirois, ${ }^{37}$ A. Zabi, ${ }^{37}$ A. Zghiche, ${ }^{37}$ J.-L. Agram, ${ }^{38, r}$ J. Andrea,${ }^{38}$ D. Bloch, ${ }^{38}$ G. Bourgatte, ${ }^{38}$ J.-M. Brom, ${ }^{38}$ E. C. Chabert, ${ }^{38}$ C. Collard ${ }^{38}$ J.-C. Fontaine, ${ }^{38, r}$ D. Gelé, ${ }^{38}$ U. Goerlach, ${ }^{38}$ C. Grimault, ${ }^{38}$ A.-C. Le Bihan, ${ }^{38}$ P. Van Hove,${ }^{38}$ E. Asilar, ${ }^{39}$ S. Beauceron, ${ }^{39}$ C. Bernet, ${ }^{39}$ G. Boudoul, ${ }^{39}$ C. Camen,${ }^{39}$ A. Carle,${ }^{39}$ N. Chanon, ${ }^{39}$ D. Contardo, ${ }^{39}$ P. Depasse, ${ }^{39}$ H. El Mamouni, ${ }^{39}$ J. Fay, ${ }^{39}$ S. Gascon, ${ }^{39}$ M. Gouzevitch, ${ }^{39}$ B. Ille, ${ }^{39}$ Sa. Jain, ${ }^{39}$ I. B. Laktineh, ${ }^{39}$ H. Lattaud ${ }^{39}$ A. Lesauvage, ${ }^{39}$ M. Lethuillier, ${ }^{39}$ L. Mirabito, ${ }^{39}$ L. Torterotot,${ }^{39}$ G. Touquet, ${ }^{39}$ M. Vander Donckt, ${ }^{39}$ S. Viret, ${ }^{39}$ I. Bagaturia, ${ }^{40, s}$ Z. Tsamalaidze, ${ }^{40,1}$ L. Feld, ${ }^{41}$ K. Klein, ${ }^{41}$ M. Lipinski, ${ }^{41}$ D. Meuser, ${ }^{41}$ A. Pauls, ${ }^{41}$ M. Preuten, ${ }^{41}$ M. P. Rauch,${ }^{41}$ J. Schulz,${ }^{41}$ M. Teroerde, ${ }^{41}$ D. Eliseev, ${ }^{42}$ M. Erdmann, ${ }^{42}$ P. Fackeldey, ${ }^{42}$ B. Fischer, ${ }^{42}$ S. Ghosh, ${ }^{42}$ T. Hebbeker, ${ }^{42}$ K. Hoepfner, ${ }^{42}$ H. Keller,${ }^{42}$ L. Mastrolorenzo, ${ }^{42}$ M. Merschmeyer, ${ }^{42}$ A. Meyer ${ }^{42}$ P. Millet, ${ }^{42}$ G. Mocellin, ${ }^{42}$ S. Mondal, ${ }^{42}$ S. Mukherjee, ${ }^{42}$ D. Noll, ${ }^{42}$ A. Novak, ${ }^{42}$ T. Pook, ${ }^{42}$ A. Pozdnyakov,${ }^{42}$ T. Quast,${ }^{42}$ M. Radziej, ${ }^{42}$ Y. Rath, ${ }^{42}$ H. Reithler, ${ }^{42}$ J. Roemer, ${ }^{42}$ A. Schmidt, ${ }^{42}$ S. C. Schuler, ${ }^{42}$ A. Sharma, ${ }^{42}$ S. Wiedenbeck, ${ }^{42}$ S. Zaleski, ${ }^{42}$ C. Dziwok, ${ }^{43}$ G. Flügge, ${ }^{43}$ W. Haj Ahmad, ${ }^{43, t}$ O. Hlushchenko, ${ }^{43}$ T. Kress, ${ }^{43}$ A. Nowack, ${ }^{43}$ C. Pistone, ${ }^{43}$ O. Pooth, ${ }^{43}$ D. Roy, ${ }^{43}$ H. Sert, ${ }^{43}$ A. Stahl, ${ }^{43, u}$ T. Ziemons, ${ }^{43}$ H. Aarup Petersen, ${ }^{44}$ M. Aldaya Martin, ${ }^{44}$ P. Asmuss, ${ }^{44}$ I. Babounikau, ${ }^{44}$ S. Baxter ${ }^{44}$ O. Behnke, ${ }^{44}$ A. Bermúdez Martínez ${ }^{44}$ A. A. Bin Anuar, ${ }^{44}$ K. Borras, ${ }^{44, v}$ V. Botta, ${ }^{44}$ D. Brunner, ${ }^{44}$ A. Campbell, ${ }^{44}$ A. Cardini, ${ }^{44}$ P. Connor, ${ }^{44}$ S. Consuegra Rodríguez, ${ }^{44}$ V. Danilov, ${ }^{44}$ A. De Wit ${ }^{44}$ M. M. Defranchis, ${ }^{44}$ L. Didukh ${ }^{44}$ D. Domínguez Damiani, ${ }^{44}$ G. Eckerlin,${ }^{44}$ D. Eckstein, ${ }^{44}$ T. Eichhorn, ${ }^{44}$ L. I. Estevez Banos, ${ }^{44}$ E. Gallo, ${ }^{44, w}$ A. Geiser ${ }^{44}$ A. Giraldi, ${ }^{44}$ A. Grohsjean,${ }^{44}$ M. Guthoff, ${ }^{44}$ A. Harb, ${ }^{44}$ A. Jafari, ${ }^{44, x}$ N. Z. Jomhari, ${ }^{44}$ H. Jung, ${ }^{44}$ A. Kasem, ${ }^{44, v}$ M. Kasemann, ${ }^{44}$ H. Kaveh, ${ }^{44}$ C. Kleinwort, ${ }^{44}$ J. Knolle, ${ }^{44}$ D. Krücker, ${ }^{44}$ W. Lange, ${ }^{44}$ T. Lenz, ${ }^{44}$ J. Lidrych, ${ }^{44}$ K. Lipka, ${ }^{44}$ W. Lohmann, ${ }^{44, y}$ R. Mankel, ${ }^{44}$ I.-A. Melzer-Pellmann, ${ }^{44}$ J. Metwally, ${ }^{44}$ A. B. Meyer, ${ }^{44}$ M. Meyer, ${ }^{44}$ M. Missiroli, ${ }^{44}$ J. Mnich, ${ }^{44}$ A. Mussgiller, ${ }^{44}$ V. Myronenko, ${ }^{44}$ Y. Otarid, ${ }^{44}$ D. Pérez Adán, ${ }^{44}$ S. K. Pflitsch, ${ }^{44}$ D. Pitzl, ${ }^{44}$ A. Raspereza, ${ }^{44}$ A. Saggio, ${ }^{44}$ A. Saibel, ${ }^{44}$ M. Savitskyi, ${ }^{44}$ V. Scheurer, ${ }^{44}$ P. Schütze, ${ }^{44}$ 
C. Schwanenberger, ${ }^{44}$ A. Singh, ${ }^{44}$ R. E. Sosa Ricardo, ${ }^{44}$ N. Tonon, ${ }^{44}$ O. Turkot, ${ }^{44}$ A. Vagnerini, ${ }^{44}$ M. Van De Klundert, ${ }^{44}$ R. Walsh ${ }^{44}$ D. Walter, ${ }^{44}$ Y. Wen, ${ }^{44}$ K. Wichmann, ${ }^{44}$ C. Wissing, ${ }^{44}$ S. Wuchterl,${ }^{44}$ O. Zenaiev, ${ }^{44}$ R. Zlebcik ${ }^{44}$ R. Aggleton, ${ }^{45}$ S. Bein, ${ }^{45}$ L. Benato, ${ }^{45}$ A. Benecke, ${ }^{45}$ K. De Leo, ${ }^{45}$ T. Dreyer, ${ }^{45}$ A. Ebrahimi ${ }^{45}$ M. Eich, ${ }^{45}$ F. Feindt, ${ }^{45}$ A. Fröhlich, ${ }^{45}$ C. Garbers, ${ }^{45}$ E. Garutti, ${ }^{45}$ P. Gunnellini, ${ }^{45}$ J. Haller, ${ }^{45}$ A. Hinzmann, ${ }^{45}$ A. Karavdina, ${ }^{45}$ G. Kasieczka, ${ }^{45}$ R. Klanner, ${ }^{45}$ R. Kogler, ${ }^{45}$ V. Kutzner, ${ }^{45}$ J. Lange, ${ }^{45}$ T. Lange, ${ }^{45}$ A. Malara, ${ }^{45}$ C. E. N. Niemeyer, ${ }^{45}$ A. Nigamova, ${ }^{45}$ K. J. Pena Rodriguez ${ }^{45}$ O. Rieger, ${ }^{45}$ P. Schleper, ${ }^{45}$ S. Schumann ${ }^{45}$ J. Schwandt ${ }^{45}$ D. Schwarz, ${ }^{45}$ J. Sonneveld ${ }^{45}$ H. Stadie, ${ }^{45}$ G. Steinbrück, ${ }^{45}$ B. Vormwald, ${ }^{45}$ I. Zoi ${ }^{45}$ M. Baselga,${ }^{46}$ S. Baur, ${ }^{46}$ J. Bechtel, ${ }^{46}$ T. Berger, ${ }^{46}$ E. Butz, ${ }^{46}$ R. Caspart, ${ }^{46}$ T. Chwalek, ${ }^{46}$ W. De Boer, ${ }^{46}$ A. Dierlamm, ${ }^{46}$ A. Droll, ${ }^{46}$ K. El Morabit, ${ }^{46}$ N. Faltermann, ${ }^{46}$ K. Flöh, ${ }^{46}$ M. Giffels, ${ }^{46}$ A. Gottmann, ${ }^{46}$ F. Hartmann, ${ }^{46, u}$ C. Heidecker, ${ }^{46}$ U. Husemann, ${ }^{46}$ M. A. Iqbal, ${ }^{46}$ I. Katkov, ${ }^{46, z}$ P. Keicher, ${ }^{46}$ R. Koppenhöfer, ${ }^{46}$ S. Maier, ${ }^{46}$ M. Metzler, ${ }^{46}$ S. Mitra, ${ }^{46}$ D. Müller, ${ }^{46}$ Th. Müller, ${ }^{46}$ M. Musich, ${ }^{46}$ G. Quast, ${ }^{46}$ K. Rabbertz, ${ }^{46}$ J. Rauser, ${ }^{46}$ D. Savoiu, ${ }^{46}$ D. Schäfer, ${ }^{46}$ M. Schnepf, ${ }^{46}$ M. Schröder, ${ }^{46}$ D. Seith, ${ }^{46}$ I. Shvetsov, ${ }^{46}$ H. J. Simonis, ${ }^{46}$ R. Ulrich, ${ }^{46}$ M. Wassmer, ${ }^{46}$ M. Weber, ${ }^{46}$ R. Wolf, ${ }^{46}$ S. Wozniewski, ${ }^{46}$ G. Anagnostou, ${ }^{47}$ P. Asenov,${ }^{47}$ G. Daskalakis, ${ }^{47}$ T. Geralis,${ }^{47}$ A. Kyriakis,${ }^{47}$ D. Loukas, ${ }^{47}$ G. Paspalaki, ${ }^{47}$ A. Stakia, ${ }^{47}$ M. Diamantopoulou, ${ }^{48}$ D. Karasavvas, ${ }^{48}$ G. Karathanasis, ${ }^{48}$ P. Kontaxakis,${ }^{48}$ C. K. Koraka, ${ }^{48}$ A. Manousakis-Katsikakis, ${ }^{48}$ A. Panagiotou, ${ }^{48}$ I. Papavergou ${ }^{48}$ N. Saoulidou, ${ }^{48}$ K. Theofilatos ${ }^{48}$ K. Vellidis, ${ }^{48}$ E. Vourliotis, ${ }^{48}$ G. Bakas, ${ }^{49}$ K. Kousouris, ${ }^{49}$ I. Papakrivopoulos, ${ }^{49}$ G. Tsipolitis ${ }^{49}$ A. Zacharopoulou, ${ }^{49}$ I. Evangelou, ${ }^{50}$ C. Foudas, ${ }^{50}$ P. Gianneios,${ }^{50}$ P. Katsoulis, ${ }^{50}$ P. Kokkas, ${ }^{50}$ S. Mallios,${ }^{50}$ K. Manitara, ${ }^{50}$ N. Manthos, ${ }^{50}$ I. Papadopoulos, ${ }^{50}$ J. Strologas, ${ }^{50}$ M. Bartók,${ }^{51, \text { aa }}$ R. Chudasama, ${ }^{51}$ M. Csanad,${ }^{51}$ M. M. A. Gadallah, ${ }^{51, b b}$ S. Lökös, ${ }^{51, c c}$ P. Major, ${ }^{51}$ K. Mandal,${ }^{51}$ A. Mehta, ${ }^{51}$ G. Pasztor, ${ }^{51}$ O. Surányi,${ }^{51}$ G. I. Veres,${ }^{51}$ G. Bencze,${ }^{52}$ C. Hajdu, ${ }^{52}$ D. Horvath,${ }^{52, d d}$ F. Sikler, ${ }^{52}$ V. Veszpremi, ${ }^{52}$ G. Vesztergombi, ${ }^{52, a, c c}$ S. Czellar, ${ }^{53}$ J. Karancsi, ${ }^{53, \text { aa }}$ J. Molnar, ${ }^{53}$ Z. Szillasi, ${ }^{53}$ D. Teyssier,${ }^{53}$ P. Raics,${ }^{54}$ Z. L. Trocsanyi, ${ }^{54}$ B. Ujvari, ${ }^{54}$ T. Csorgo, ${ }^{55}$ F. Nemes, ${ }^{55}$ T. Novak, ${ }^{55}$ S. Choudhury,${ }^{56}$ J. R. Komaragiri, ${ }^{56}$ D. Kumar, ${ }^{56}$ L. Panwar, ${ }^{56}$ P. C. Tiwari, ${ }^{56}$ S. Bahinipati, ${ }^{57, e e}$ D. Dash, ${ }^{57}$ C. Kar,${ }^{57}$ P. Mal, ${ }^{57}$ T. Mishra, ${ }^{57}$

V. K. Muraleedharan Nair Bindhu, ${ }^{57}$ A. Nayak, ${ }^{57 f f}$ D. K. Sahoo, ${ }^{57, e e}$ N. Sur, ${ }^{57}$ S. K. Swain, ${ }^{57}$ S. Bansal, ${ }^{58}$ S. B. Beri, ${ }^{58}$ V. Bhatnagar, ${ }^{58}$ S. Chauhan, ${ }^{58}$ N. Dhingra, ${ }^{58, g g}$ R. Gupta, ${ }^{58}$ A. Kaur, ${ }^{58}$ S. Kaur, ${ }^{58}$ P. Kumari, ${ }^{58}$ M. Lohan, ${ }^{58}$ M. Meena,${ }^{58}$ K. Sandeep, ${ }^{58}$ S. Sharma, ${ }^{58}$ J. B. Singh, ${ }^{58}$ A. K. Virdi, ${ }^{58}$ A. Ahmed, ${ }^{59}$ A. Bhardwaj, ${ }^{59}$ B. C. Choudhary, ${ }^{59}$ R. B. Garg, ${ }^{59}$ M. Gola, ${ }^{59}$ S. Keshri, ${ }^{59}$ A. Kumar, ${ }^{59}$ M. Naimuddin, ${ }^{59}$ P. Priyanka, ${ }^{59}$ K. Ranjan, ${ }^{59}$ A. Shah, ${ }^{59}$ M. Bharti, ${ }^{60, h h}$

R. Bhattacharya, ${ }^{60} \mathrm{~S}$. Bhattacharya, ${ }^{60} \mathrm{D}$. Bhowmik, ${ }^{60} \mathrm{~S}$. Dutta, ${ }^{60} \mathrm{~S}$. Ghosh, ${ }^{60}$ B. Gomber, ${ }^{60, \mathrm{ii}} \mathrm{M}$. Maity, ${ }^{60, j \mathrm{j}} \mathrm{S}$. Nandan, ${ }^{60}$ P. Palit, ${ }^{60}$ A. Purohit,${ }^{60}$ P. K. Rout, ${ }^{60}$ G. Saha,${ }^{60}$ S. Sarkar,${ }^{60}$ M. Sharan, ${ }^{60}$ B. Singh ${ }^{60, \text { hh }}$ S. Thakur,${ }^{60, \text { hh }}$ P. K. Behera, ${ }^{61}$ S. C. Behera, ${ }^{61}$ P. Kalbhor, ${ }^{61}$ A. Muhammad, ${ }^{61}$ R. Pradhan,${ }^{61}$ P. R. Pujahari, ${ }^{61}$ A. Sharma, ${ }^{61}$ A. K. Sikdar, ${ }^{61}$ D. Dutta, ${ }^{62}$ V. Kumar, ${ }^{62}$ K. Naskar, ${ }^{62, k k}$ P. K. Netrakanti, ${ }^{62}$ L. M. Pant, ${ }^{62}$ P. Shukla, ${ }^{62}$ T. Aziz,${ }^{63}$ M. A. Bhat, ${ }^{63}$ S. Dugad,${ }^{63}$ R. Kumar Verma, ${ }^{63}$ U. Sarkar, ${ }^{63}$ S. Banerjee,${ }^{64}$ S. Bhattacharya,${ }^{64}$ S. Chatterjee, ${ }^{64}$ P. Das, ${ }^{64}$ M. Guchait, ${ }^{64}$ S. Karmakar,${ }^{64}$ S. Kumar, ${ }^{64}$ G. Majumder, ${ }^{64}$ K. Mazumdar, ${ }^{64}$ S. Mukherjee, ${ }^{64}$ D. Roy, ${ }^{64}$ N. Sahoo,${ }^{64}$ S. Dube, ${ }^{65}$ B. Kansal, ${ }^{65}$ A. Kapoor,${ }^{65}$ K. Kothekar, ${ }^{65}$ S. Pandey, ${ }^{65}$ A. Rane, ${ }^{65}$ A. Rastogi ${ }^{65}$ S. Sharma ${ }^{65}$ H. Bakhshiansohi, ${ }^{66,11}$ S. Chenarani, ${ }^{67, m m}$ S. M. Etesami, ${ }^{67}$ M. Khakzad, ${ }^{67}$ M. Mohammadi Najafabadi, ${ }^{67}$ M. Felcini, ${ }^{68}$ M. Grunewald, ${ }^{68}$ M. Abbrescia, ${ }^{69 a, 69 b}$ R. Aly, ${ }^{6 a, 69 b, n n}$ C. Aruta, ${ }^{69 a, 69 \mathrm{~b}}$ A. Colaleo, ${ }^{69 \mathrm{a}}$ D. Creanza, ${ }^{69 \mathrm{a}, 69 \mathrm{c}}$ N. De Filippis, ${ }^{69 a, 69 \mathrm{c}}$ M. De Palma, ${ }^{69 \mathrm{a}, 69 \mathrm{~b}}$ A. Di Florio, ${ }^{69 \mathrm{a}, 69 \mathrm{~b}}$ A. Di Pilato ${ }^{69 a, 69 b}$ W. Elmetenawee ${ }^{69 a, 69 b}$ L. Fiore,${ }^{69 a}$ A. Gelmi ${ }^{69 a, 69 b}$ M. Gul,${ }^{69 a}$ G. Iaselli,${ }^{69 a, 69 c}$ M. Ince,${ }^{69 a, 69 b}$ S. Lezki, ${ }^{69 a, 69 b}$ G. Maggi, ${ }^{69 a, 69 c}$ M. Maggi, ${ }^{69 a}$ I. Margjeka, ${ }^{69 a, 69 b}$ J. A. Merlin, ${ }^{69 a}$ S. My, ${ }^{69 a, 69 b}$ S. Nuzzo, ${ }^{69 a, 69 b}$ A. Pompili, ${ }^{69,69 b}$ G. Pugliese, ${ }^{69 a, 69 c}$ A. Ranieri, ${ }^{69 a}$ G. Selvaggi, ${ }^{69,69 b}$ L. Silvestris, ${ }^{69 a}$ F. M. Simone,${ }^{69 a, 69 b}$ R. Venditti, ${ }^{69 a}$ P. Verwilligen, ${ }^{69 \mathrm{a}}$ G. Abbiendi, ${ }^{70 \mathrm{a}}$ C. Battilana, ${ }^{70,70 \mathrm{~b}}$ D. Bonacorsi, ${ }^{70 a}, 70 \mathrm{~b}$ L. Borgonovi, ${ }^{70 \mathrm{a}, 70 \mathrm{~b}}$ S. Braibant-Giacomelli, ${ }^{70 \mathrm{a}, 70 \mathrm{~b}}$ R. Campanini ${ }^{70 a, 70 b}$ P. Capiluppi, ${ }^{70 a, 70 b}$ A. Castro, ${ }^{70 a, 70 b}$ F. R. Cavallo, ${ }^{70 a}$ M. Cuffiani, ${ }^{70 a, 70 b}$ G. M. Dallavalle, ${ }^{70 a}$ T. Diotalevi, ${ }^{70 a, 70 b}$ F. Fabbri, ${ }^{70 a}$ A. Fanfani, ${ }^{70,70 b}$ E. Fontanesi, ${ }^{70 a, 70 b}$ P. Giacomelli, ${ }^{70 a}$ L. Giommi, ${ }^{70 a}, 70 b$ C. Grandi, ${ }^{70 a}$ L. Guiducci, ${ }^{70 a, 70 b}$ F. Iemmi, ${ }^{70 a, 70 b}$ S. Lo Meo, ${ }^{70 a, 0 o}$ S. Marcellini, ${ }^{70 a}$ G. Masetti, ${ }^{70 a}$ F. L. Navarria, ${ }^{70 a, 70 b}$ A. Perrotta, ${ }^{70 a}$ F. Primavera, ${ }^{70 a, 70 b}$ A. M. Rossi, ${ }^{70 a, 70 b}$ T. Rovelli, ${ }^{70 a, 70 b}$ G. P. Siroli, ${ }^{70 a, 70 b}$ N. Tosi, ${ }^{70 a}$ S. Albergo, ${ }^{71 a, 71 b, p p}$ S. Costa, ${ }^{71 a, 71 b}$ A. Di Mattia, ${ }^{71 a}$ R. Potenza, ${ }^{71 a, 71 b}$ A. Tricomi, ${ }^{71 a, 71 b, p p}$ C. Tuve, ${ }^{71 a, 71 b}$ G. Barbagli, ${ }^{72 a}$ A. Cassese, ${ }^{72 a}$ R. Ceccarelli, ${ }^{72 a, 72 b}$ V. Ciulli, ${ }^{72 a, 72 b}$ C. Civinini, ${ }^{72 a}$ R. D’Alessandro, ${ }^{72 a, 72 b}$ F. Fiori, ${ }^{72 a}$ E. Focardi, ${ }^{72 a, 72 b}$ G. Latino, ${ }^{72 a, 72 b}$ P. Lenzi, ${ }^{72 a, 72 b}$ M. Lizzo, ${ }^{72 a, 72 b}$ M. Meschini, ${ }^{72 a}$ S. Paoletti, ${ }^{72 a}$ R. Seidita ${ }^{72 a, 72 b}$ G. Sguazzoni, ${ }^{72 a}$ L. Viliani, ${ }^{72 a}$ L. Benussi,${ }^{73}$ S. Bianco, ${ }^{73}$ D. Piccolo, ${ }^{73}$ M. Bozzo, ${ }^{74 a}, 74 \mathrm{~b}$ F. Ferro, ${ }^{74 a}$ R. Mulargia, ${ }^{74 a, 74 b}$ E. Robutti, ${ }^{74 a}$ S. Tosi, ${ }^{74 a, 74 b}$ A. Benaglia, ${ }^{75 a}$ A. Beschi, ${ }^{75 a}, 75 b$ F. Brivio, ${ }^{75 a, 75 b}$ F. Cetorelli, ${ }^{75 a, 75 b}$ V. Ciriolo, ${ }^{75 a, 75 b, u}$ F. De Guio, ${ }^{75 a, 75 b}$ M. E. Dinardo, ${ }^{75 a, 75 b}$ P. Dini, ${ }^{75 a}$ S. Gennai, ${ }^{75 a}$ A. Ghezzi, ${ }^{75 a, 75 b}$ P. Govoni, ${ }^{75 a, 75 b}$ L. Guzzi, ${ }^{75 a, 75 b}$ M. Malberti, ${ }^{75 a}$ S. Malvezzi ${ }^{75 a}$ D. Menasce, ${ }^{75 a}$ F. Monti ${ }^{75 a, 75 b}$ L. Moroni, ${ }^{75 a}$ M. Paganoni, ${ }^{75 a, 75 b}$ D. Pedrini, ${ }^{75 a}$ S. Ragazzi, ${ }^{75 a, 75 b}$ T. Tabarelli de Fatis, ${ }^{75 a, 75 b}$ D. Valsecchi, ${ }^{75 a, 75 b, u}$ 
D. Zuolo, ${ }^{75 a, 75 b}$ S. Buontempo, ${ }^{76 a}$ N. Cavallo, ${ }^{76 a, 76 c}$ A. De Iorio, ${ }^{76 a, 76 b}$ F. Fabozzi, ${ }^{76 a, 76 c}$ F. Fienga, ${ }^{76 a}$ A. O. M. Iorio, ${ }^{76 a, 76 b}$ L. Layer, ${ }^{76 a, 76 b}$ L. Lista, ${ }^{76 a, 76 b}$ S. Meola, ${ }^{76 a, 76 d, u}$ P. Paolucci, ${ }^{76 a, u}$ B. Rossi, ${ }^{76 a}$ C. Sciacca, ${ }^{76 a, 76 b}$ E. Voevodina, ${ }^{76 a, 76 b}$ P. Azzi, ${ }^{77 a}$ N. Bacchetta, ${ }^{77 a}$ A. Boletti, ${ }^{77 a, 77 b}$ A. Bragagnolo, ${ }^{77,77 b}$ R. Carlin, ${ }^{77 a, 77 b}$ P. Checchia, ${ }^{77 a}$ P. De Castro Manzano, ${ }^{77 a}$ T. Dorigo, ${ }^{77 \mathrm{a}}$ F. Gasparini, ${ }^{77 \mathrm{a}, 77 \mathrm{~b}}$ U. Gasparini, ${ }^{77 \mathrm{a}, 77 \mathrm{~b}}$ S. Y. Hoh, ${ }^{77,77 \mathrm{~b}}$ M. Margoni, ${ }^{77 a, 77 \mathrm{~b}}$ A. T. Meneguzzo, ${ }^{77 a, 77 \mathrm{~b}}$ M. Presilla ${ }^{77 a, 77 b}$ P. Ronchese, ${ }^{77 a, 77 b}$ R. Rossin, ${ }^{77 a, 77 b}$ F. Simonetto, ${ }^{77 a, 77 b}$ G. Strong, ${ }^{77 a}$ A. Tiko, ${ }^{77 a}$ M. Tosi, ${ }^{77 a, 77 b}$ H. YARAR, ${ }^{77 a, 77 b}$ M. Zanetti, ${ }^{77 a, 77 b}$ P. Zotto, ${ }^{77 a, 77 b}$ A. Zucchetta, ${ }^{77 a, 77 b}$ G. Zumerle, ${ }^{77 a, 77 b}$ A. Braghieri, ${ }^{78 a}$ S. Calzaferri, ${ }^{78 a, 78 b}$ D. Fiorina, ${ }^{78 a, 78 b}$ P. Montagna, ${ }^{78 a, 78 b}$ S. P. Ratti, ${ }^{78 a, 78 b}$ V. Re ${ }^{78 a}$ M. Ressegotti, ${ }^{78 a, 78 b}$ C. Riccardi, ${ }^{78 a, 78 b}$ P. Salvini, ${ }^{78 a}$ I. Vai, ${ }^{78 a}$ P. Vitulo, ${ }^{78 a, 78 b}$ M. Biasini, ${ }^{79 a, 79 b}$ G. M. Bilei, ${ }^{79 a}$ D. Ciangottini, ${ }^{79 a, 79 b}$ L. Fanò,${ }^{79 a, 79 b}$ P. Lariccia, ${ }^{79 a, 79 b}$ G. Mantovani, ${ }^{79 a, 79 b}$ V. Mariani, ${ }^{79 a, 79 b}$ M. Menichelli, ${ }^{79 a}$ F. Moscatelli, ${ }^{79 a}$ A. Rossi, ${ }^{79 a, 79 b}$ A. Santocchia, ${ }^{79 a, 79 b}$ D. Spiga, ${ }^{79 a}$ T. Tedeschi, ${ }^{79,79 b}$ K. Androsov, ${ }^{80 a}$ P. Azzurri, ${ }^{80 a}$ G. Bagliesi, ${ }^{80 a}$ V. Bertacchi, ${ }^{80 a, 80 c}$ L. Bianchini, ${ }^{80 a}$ T. Boccali, ${ }^{80 a}$ R. Castaldi, ${ }^{80 a}$ M. A. Ciocci, ${ }^{80 a, 80 b}$ R. Dell'Orso, ${ }^{80 a}$ M. R. Di Domenico, ${ }^{80 a, 80 b}$ S. Donato ${ }^{80 a}$ L. Giannini, ${ }^{80 a, 80 c}$ A. Giassi, ${ }^{80 a}$ M. T. Grippo, ${ }^{80 a}$ F. Ligabue ${ }^{80 a, 80 c}$ E. Manca, ${ }^{80 a, 80 c}$ G. Mandorli, ${ }^{80,80 c}$ A. Messineo, ${ }^{80 a, 80 b}$ F. Palla, ${ }^{80 a}$ G. Ramirez-Sanchez, ${ }^{80,80 c}$ A. Rizzi, ${ }^{80 a 80 b}$ G. Rolandi, ${ }^{80 a, 80 c}$ S. Roy Chowdhury, ${ }^{80 a, 80 c}$ A. Scribano,${ }^{80 a}$ N. Shafiei, ${ }^{80 a, 80 b}$ P. Spagnolo, ${ }^{80 a}$ R. Tenchini, ${ }^{80 a}$ G. Tonelli, ${ }^{80 a, 80 b}$ N. Turini, ${ }^{80 a}$ A. Venturi, ${ }^{80 a}$ P. G. Verdini, ${ }^{80 a}$ F. Cavallari, ${ }^{81 a}$ M. Cipriani, ${ }^{81 a, 81 b}$ D. Del Re,${ }^{81 a, 81 b}$ E. Di Marco,${ }^{81 \mathrm{a}}$ M. Diemoz, ${ }^{81 \mathrm{a}}$ E. Longo, ${ }^{81 \mathrm{a}, 81 \mathrm{~b}}$ P. Meridiani, ${ }^{81 \mathrm{a}}$ G. Organtini,${ }^{81 \mathrm{a}, 81 \mathrm{~b}}$ F. Pandolfi, ${ }^{81 \mathrm{a}}$ R. Paramatti, ${ }^{81,81 \mathrm{~b}}$ C. Quaranta, ${ }^{81 a, 81 b}$ S. Rahatlou, ${ }^{81 a, 81 b}$ C. Rovelli, ${ }^{81 a}$ F. Santanastasio, ${ }^{81 a, 81 b}$ L. Soffi, ${ }^{81 a, 81 b}$ R. Tramontano, ${ }^{81 a, 81 b}$ N. Amapane, ${ }^{82 a, 82 b}$ R. Arcidiacono, ${ }^{82 a, 82 c}$ S. Argiro, ${ }^{82 a, 82 b}$ M. Arneodo, ${ }^{82 a, 82 c}$ N. Bartosik, ${ }^{82 a}$ R. Bellan, ${ }^{82 a, 82 b}$ A. Bellora, ${ }^{82 a, 82 b}$ C. Biino, ${ }^{82 a}$ A. Cappati, ${ }^{82 a, 82 b}$ N. Cartiglia, ${ }^{82 a}$ S. Cometti, ${ }^{82 a}$ M. Costa, ${ }^{82 a, 82 b}$ R. Covarelli, ${ }^{82 a, 82 b}$ N. Demaria, ${ }^{82 a}$ B. Kiani, ${ }^{82 a, 82 b}$ F. Legger, ${ }^{82 a}$ C. Mariotti, ${ }^{82 a}$ S. Maselli, ${ }^{82 a}$ E. Migliore, ${ }^{82 a, 82 b}$ V. Monaco, ${ }^{82 a, 82 b}$ E. Monteil, ${ }^{82 a, 82 b}$ M. Monteno, ${ }^{82 a}$ M. M. Obertino, ${ }^{82 a, 82 b}$ G. Ortona, ${ }^{82 a}$ L. Pacher, ${ }^{82 a, 82 b}$ N. Pastrone, ${ }^{82 a}$ M. Pelliccioni, ${ }^{82 a}$ G. L. Pinna Angioni, ${ }^{82 a, 82 b}$ M. Ruspa, ${ }^{82 a, 82 c}$ R. Salvatico, ${ }^{82 a, 82 b}$ F. Siviero, ${ }^{82 a, 82 b}$ V. Sola, ${ }^{82 a}$ A. Solano, ${ }^{82 a, 82 b}$ D. Soldi, ${ }^{82 a, 82 b}$ A. Staiano, ${ }^{82 a}$ D. Trocino, ${ }^{82 a, 82 b}$ S. Belforte, ${ }^{83 a}$ V. Candelise,${ }^{83 a, 83 b}$ M. Casarsa, ${ }^{83 a}$ F. Cossutti, ${ }^{83 a}$ A. Da Rold, ${ }^{83 a, 83 b}$ G. Della Ricca, ${ }^{83 a, 83 b}$ F. Vazzoler, ${ }^{83 a, 83 b}$ S. Dogra ${ }^{84}$ C. Huh, ${ }^{84}$ B. Kim, ${ }^{84}$ D. H. Kim,${ }^{84}$ G. N. Kim, ${ }^{84}$ J. Lee,${ }^{84}$ S. W. Lee ${ }^{84}$ C. S. Moon ${ }^{84}$ Y. D. Oh ${ }^{84}$ S. I. Pak ${ }^{84}$ B. C. Radburn-Smith,${ }^{84}$ S. Sekmen ${ }^{84}$ Y. C. Yang, ${ }^{84}$ H. Kim, ${ }^{85}$ D. H. Moon, ${ }^{85}$ B. Francois, ${ }^{86}$ T. J. Kim, ${ }^{86}$ J. Park, ${ }^{86}$ S. Cho, ${ }^{87}$ S. Choi,${ }^{87}$ Y. Go, ${ }^{87}$ S. Ha ${ }^{87}$ B. Hong,${ }^{87}$ K. Lee, ${ }^{87}$ K. S. Lee,${ }^{87}$ J. Lim, ${ }^{87}$ J. Park, ${ }^{87}$ S. K. Park, ${ }^{87}$ J. Yoo,${ }^{87}$ J. Goh, ${ }^{88}$ A. Gurtu, ${ }^{88}$ H. S. Kim,${ }^{89}$ Y. Kim, ${ }^{89}$ J. Almond, ${ }^{90}$ J. H. Bhyun, ${ }^{90}$ J. Choi, ${ }^{90}$ S. Jeon, ${ }^{90}$ J. Kim, ${ }^{90}$ J. S. Kim, ${ }^{90}$ S. Ko, ${ }^{90}$ H. Kwon, ${ }^{90}$ H. Lee, ${ }^{90}$ K. Lee, ${ }^{90}$ S. Lee, ${ }^{90}$ K. Nam,${ }^{90}$ B. H. Oh, ${ }^{90}$ M. Oh, ${ }^{90}$ S. B. Oh, ${ }^{90}$ H. Seo, ${ }^{90}$ U. K. Yang, ${ }^{90}$ I. Yoon, ${ }^{90}$ D. Jeon, ${ }^{91}$ J. H. Kim, ${ }^{91}$ B. Ko, ${ }^{91}$ J. S. H. Lee, ${ }^{91}$ I. C. Park,,${ }^{91}$ Y. Roh, ${ }^{91}$ D. Song, ${ }^{91}$ I. J. Watson, ${ }^{91}$ H. D. Yoo, ${ }^{92}$ Y. Choi,${ }^{93}$ C. Hwang, ${ }^{93}$ Y. Jeong, ${ }^{93}$ H. Lee, ${ }^{93}$ Y. Lee, ${ }^{93}$ I. Yu, ${ }^{93}$ Y. Maghrbi, ${ }^{94}$ V. Veckalns, ${ }^{95, q 9}$ A. Juodagalvis, ${ }^{96}$ A. Rinkevicius, ${ }^{96}$ G. Tamulaitis, ${ }^{96}$ W. A. T. Wan Abdullah, ${ }^{97}$ M. N. Yusli, ${ }^{97}$ Z. Zolkapli, ${ }^{97}$ J. F. Benitez, ${ }^{98}$

A. Castaneda Hernandez, ${ }^{98}$ J. A. Murillo Quijada, ${ }^{98}$ L. Valencia Palomo, ${ }^{98}$ H. Castilla-Valdez, ${ }^{99}$ E. De La Cruz-Burelo, ${ }^{99}$ I. Heredia-De La Cruz, ${ }^{99, \text { rr }}$ R. Lopez-Fernandez, ${ }^{99}$ A. Sanchez-Hernandez, ${ }^{99}$ S. Carrillo Moreno, ${ }^{100}$ C. Oropeza Barrera, ${ }^{100}$ M. Ramirez-Garcia, ${ }^{100}$ F. Vazquez Valencia,${ }^{100}$ J. Eysermans, ${ }^{101}$ I. Pedraza, ${ }^{101}$ H. A. Salazar Ibarguen, ${ }^{101}$

C. Uribe Estrada, ${ }^{101}$ A. Morelos Pineda, ${ }^{102}$ J. Mijuskovic, ${ }^{103, e}$ N. Raicevic, ${ }^{103}$ D. Krofcheck, ${ }^{104}$ S. Bheesette, ${ }^{105}$ P. H. Butler, ${ }^{105}$ A. Ahmad, ${ }^{106}$ M. I. Asghar, ${ }^{106}$ M. I. M. Awan, ${ }^{106}$ H. R. Hoorani, ${ }^{106}$ W. A. Khan, ${ }^{106}$ M. A. Shah, ${ }^{106}$ M. Shoaib, ${ }^{106}$ M. Waqas, ${ }^{106}$ V. Avati, ${ }^{107}$ L. Grzanka, ${ }^{107}$ M. Malawski, ${ }^{107}$ H. Bialkowska, ${ }^{108}$ M. Bluj, ${ }^{108}$ B. Boimska, ${ }^{108}$ T. Frueboes, ${ }^{108}$ M. Górski, ${ }^{108}$ M. Kazana, ${ }^{108}$ M. Szleper, ${ }^{108}$ P. Traczyk, ${ }^{108}$ P. Zalewski, ${ }^{108}$ K. Bunkowski, ${ }^{109}$ A. Byszuk, ${ }^{109, s s}$ K. Doroba, ${ }^{109}$ A. Kalinowski, ${ }^{109}$ M. Konecki, ${ }^{109}$ J. Krolikowski, ${ }^{109}$ M. Olszewski, ${ }^{109}$ M. Walczak, ${ }^{109}$ M. Araujo, ${ }^{110}$ P. Bargassa, ${ }^{110}$ D. Bastos, ${ }^{110}$ P. Faccioli, ${ }^{110}$ M. Gallinaro, ${ }^{110}$ J. Hollar,${ }^{110}$ N. Leonardo, ${ }^{110}$ T. Niknejad,${ }^{110}$ J. Seixas, ${ }^{110}$ K. Shchelina, ${ }^{110}$ O. Toldaiev, ${ }^{110}$ J. Varela, ${ }^{110}$ S. Afanasiev, ${ }^{111}$ P. Bunin,,${ }^{111}$ Y. Ershov, ${ }^{111}$ M. Gavrilenko, ${ }^{111}$ A. Golunov, ${ }^{111}$ I. Golutvin, ${ }^{111}$ N. Gorbounov, ${ }^{111}$ I. Gorbunov, ${ }^{111}$ V. Karjavine, ${ }^{111}$ A. Lanev, ${ }^{111}$ A. Malakhov, ${ }^{111}$ V. Matveev, ${ }^{11, t t, u u}$ P. Moisenz, ${ }^{111}$ V. Palichik, ${ }^{111}$ V. Perelygin, ${ }^{111}$ M. Savina, ${ }^{111}$ S. Shmatov, ${ }^{111}$ S. Shulha,${ }^{111}$ V. Smirnov, ${ }^{111}$ O. Teryaev, ${ }^{111}$ N. Voytishin, ${ }^{111}$ B. S. Yuldashev, ${ }^{111, v v}$ A. Zarubin, ${ }^{111}$ G. Gavrilov, ${ }^{112}$ V. Golovtcov, ${ }^{112}$ Y. Ivanov, ${ }^{112}$ V. Kim, ${ }^{112 \text {,ww }}$ E. Kuznetsova, ${ }^{112, x x}$ V. Murzin, ${ }^{112}$ V. Oreshkin, ${ }^{112}$ I. Smirnov, ${ }^{112}$ D. Sosnov, ${ }^{112}$ V. Sulimov, ${ }^{112}$ L. Uvarov, ${ }^{112}$ S. Volkov, ${ }^{112}$ A. Vorobyev, ${ }^{112}$ Yu. Andreev, ${ }^{113}$ A. Dermenev, ${ }^{113}$ S. Gninenko, ${ }^{113}$ N. Golubev, ${ }^{113}$ A. Karneyeu, ${ }^{113}$ M. Kirsanov, ${ }^{113}$ N. Krasnikov, ${ }^{113}$ A. Pashenkov, ${ }^{113}$ G. Pivovarov,${ }^{113}$ D. Tlisov, ${ }^{113, a}$ A. Toropin, ${ }^{113}$ V. Epshteyn, ${ }^{114}$ V. Gavrilov, ${ }^{114}$ N. Lychkovskaya, ${ }^{114}$ A. Nikitenko, ${ }^{114, y y}$ V. Popov, ${ }^{114}$ G. Safronov, ${ }^{114}$ A. Spiridonov, ${ }^{114}$ A. Stepennov, ${ }^{114}$ M. Toms, ${ }^{114}$ E. Vlasov, ${ }^{114}$ A. Zhokin, ${ }^{114}$ T. Aushev, ${ }^{115}$ O. Bychkova, ${ }^{116}$ M. Chadeeva, ${ }^{116, z z}$ D. Philippov, ${ }^{116}$ E. Popova,${ }^{116}$ V. Rusinov, ${ }^{116}$ V. Andreev, ${ }^{117}$ M. Azarkin, ${ }^{117}$ I. Dremin, ${ }^{117}$ M. Kirakosyan, ${ }^{117}$ A. Terkulov, ${ }^{117}$ A. Baskakov, ${ }^{118}$ A. Belyaev, ${ }^{118}$ E. Boos, ${ }^{118}$ 
V. Bunichev, ${ }^{118}$ M. Dubinin, ${ }^{118, a a a}$ L. Dudko, ${ }^{118}$ A. Ershov, ${ }^{118}$ A. Gribushin, ${ }^{118}$ V. Klyukhin, ${ }^{118}$ O. Kodolova, ${ }^{118}$ I. Lokhtin, ${ }^{118}$ S. Obraztsov, ${ }^{118}$ V. Savrin, ${ }^{118}$ V. Blinov, ${ }^{119, b b b}$ T. Dimova, ${ }^{119, b b b}$ L. Kardapoltsev, ${ }^{119, \text { bbb }}$ I. Ovtin, ${ }^{119, \text { bbb }}$ Y. Skovpen, ${ }^{119, \text { bbb }}$ I. Azhgirey, ${ }^{120}$ I. Bayshev, ${ }^{120}$ V. Kachanov, ${ }^{120}$ A. Kalinin, ${ }^{120}$ D. Konstantinov, ${ }^{120}$ V. Petrov, ${ }^{120}$ R. Ryutin, ${ }^{120}$ A. Sobol, ${ }^{120}$ S. Troshin, ${ }^{120}$ N. Tyurin, ${ }^{120}$ A. Uzunian, ${ }^{120}$ A. Volkov, ${ }^{120}$ A. Babaev, ${ }^{121}$ A. Iuzhakov, ${ }^{121}$ V. Okhotnikov, ${ }^{121}$ L. Sukhikh, ${ }^{121}$ V. Borchsh, ${ }^{122}$ V. Ivanchenko, ${ }^{122}$ E. Tcherniaev, ${ }^{122}$ P. Adzic, ${ }^{123, \text { ccc }}$ P. Cirkovic, ${ }^{123}$ M. Dordevic, ${ }^{123}$ P. Milenovic, ${ }^{123}$ J. Milosevic, ${ }^{123}$ M. Aguilar-Benitez, ${ }^{124}$ J. Alcaraz Maestre, ${ }^{124}$ A. Álvarez Fernández, ${ }^{124}$

I. Bachiller, ${ }^{124}$ M. Barrio Luna, ${ }^{124}$ Cristina F. Bedoya, ${ }^{124}$ J. A. Brochero Cifuentes, ${ }^{124}$ C. A. Carrillo Montoya, ${ }^{124}$ M. Cepeda, ${ }^{124}$ M. Cerrada, ${ }^{124}$ N. Colino, ${ }^{124}$ B. De La Cruz, ${ }^{124}$ A. Delgado Peris, ${ }^{124}$ J. P. Fernández Ramos, ${ }^{124}$ J. Flix, ${ }^{124}$ M. C. Fouz, ${ }^{124}$ A. García Alonso, ${ }^{124}$ O. Gonzalez Lopez, ${ }^{124}$ S. Goy Lopez, ${ }^{124}$ J. M. Hernandez, ${ }^{124}$ M. I. Josa, ${ }^{124}$ J. León Holgado, ${ }^{124}$ D. Moran, ${ }^{124}$ Á. Navarro Tobar, ${ }^{124}$ A. Pérez-Calero Yzquierdo, ${ }^{124}$ J. Puerta Pelayo, ${ }^{124}$ I. Redondo, ${ }^{124}$ L. Romero, ${ }^{124}$ S. Sánchez Navas, ${ }^{124}$ M. S. Soares, ${ }^{124}$ A. Triossi, ${ }^{124}$ L. Urda Gómez, ${ }^{124}$ C. Willmott, ${ }^{124}$ C. Albajar, ${ }^{125}$ J. F. de Trocóniz, ${ }^{125}$ R. Reyes-Almanza, ${ }^{125}$ B. Alvarez Gonzalez, ${ }^{126}$ J. Cuevas, ${ }^{126}$ C. Erice, ${ }^{126}$ J. Fernandez Menendez, ${ }^{126}$ S. Folgueras, ${ }^{126}$ I. Gonzalez Caballero, ${ }^{126}$ E. Palencia Cortezon, ${ }^{126}$ C. Ramón Álvarez, ${ }^{126}$ J. Ripoll Sau, ${ }^{126}$ V. Rodríguez Bouza, ${ }^{126}$ S. Sanchez Cruz, ${ }^{126}$ A. Trapote, ${ }^{126}$ I. J. Cabrillo, ${ }^{127}$ A. Calderon, ${ }^{127}$ B. Chazin Quero, ${ }^{127}$ J. Duarte Campderros, ${ }^{127}$ M. Fernandez, ${ }^{127}$ P. J. Fernández Manteca, ${ }^{127}$ G. Gomez, ${ }^{127}$ C. Martinez Rivero, ${ }^{127}$ P. Martinez Ruiz del Arbol, ${ }^{127}$ F. Matorras, ${ }^{127}$ J. Piedra Gomez, ${ }^{127}$ C. Prieels, ${ }^{127}$ F. Ricci-Tam, ${ }^{127}$ T. Rodrigo, ${ }^{127}$ A. Ruiz-Jimeno, ${ }^{127}$ L. Scodellaro, ${ }^{127}$ I. Vila, ${ }^{127}$ J. M. Vizan Garcia, ${ }^{127}$ MK Jayananda, ${ }^{128}$ B. Kailasapathy, ${ }^{128, d d d}$ D. U. J. Sonnadara, ${ }^{128}$ DDC Wickramarathna, ${ }^{128}$ W. G. D. Dharmaratna, ${ }^{129}$ K. Liyanage,${ }^{129}$ N. Perera, ${ }^{129}$ N. Wickramage, ${ }^{129}$ T. K. Aarrestad, ${ }^{130}$ D. Abbaneo, ${ }^{130}$ B. Akgun, ${ }^{130}$ E. Auffray, ${ }^{130}$ G. Auzinger, ${ }^{130}$ J. Baechler, ${ }^{130}$ P. Baillon, ${ }^{130}$ A. H. Ball, ${ }^{130}$ D. Barney, ${ }^{130}$ J. Bendavid, ${ }^{130}$ N. Beni, ${ }^{130}$ M. Bianco, ${ }^{130}$ A. Bocci, ${ }^{130}$ P. Bortignon, ${ }^{130}$ E. Bossini, ${ }^{130}$ E. Brondolin, ${ }^{130}$ T. Camporesi, ${ }^{130}$ G. Cerminara, ${ }^{130}$ L. Cristella, ${ }^{130}$ D. d'Enterria, ${ }^{130}$ A. Dabrowski, ${ }^{130}$ N. Daci, ${ }^{130}$ V. Daponte, ${ }^{130}$ A. David, ${ }^{130}$ A. De Roeck, ${ }^{130}$ M. Deile, ${ }^{130}$ R. Di Maria, ${ }^{130}$ M. Dobson, ${ }^{130}$ M. Dünser, ${ }^{130}$ N. Dupont, ${ }^{130}$ A. Elliott-Peisert, ${ }^{130}$ N. Emriskova, ${ }^{130}$ F. Fallavollita, ${ }^{130, e e e}$ D. Fasanella, ${ }^{130}$ S. Fiorendi, ${ }^{130}$ G. Franzoni, ${ }^{130}$ J. Fulcher, ${ }^{130}$ W. Funk, ${ }^{130}$ S. Giani, ${ }^{130}$ D. Gigi, ${ }^{130}$ K. Gill, ${ }^{130}$ F. Glege, ${ }^{130}$ L. Gouskos, ${ }^{130}$ M. Guilbaud, ${ }^{130}$ D. Gulhan, ${ }^{130}$ M. Haranko, ${ }^{130}$ J. Hegeman, ${ }^{130}$ Y. Iiyama, ${ }^{130}$ V. Innocente, ${ }^{130}$ T. James, ${ }^{130}$ P. Janot, ${ }^{130}$ J. Kaspar, ${ }^{130}$ J. Kieseler, ${ }^{130}$ M. Komm, ${ }^{130}$ N. Kratochwil, ${ }^{130}$ C. Lange, ${ }^{130}$ P. Lecoq, ${ }^{130}$ K. Long, ${ }^{130}$ C. Lourenço, ${ }^{130}$ L. Malgeri, ${ }^{130}$ M. Mannelli, ${ }^{130}$ A. Massironi, ${ }^{130}$ F. Meijers, ${ }^{130}$ S. Mersi, ${ }^{130}$ E. Meschi, ${ }^{130}$ F. Moortgat, ${ }^{130}$ M. Mulders, ${ }^{130}$ J. Ngadiuba, ${ }^{130}$ J. Niedziela, ${ }^{130}$ S. Orfanelli, ${ }^{130}$ L. Orsini, ${ }^{130}$ F. Pantaleo, ${ }^{130, u}$ L. Pape, ${ }^{130}$ E. Perez, ${ }^{130}$ M. Peruzzi, ${ }^{130}$ A. Petrilli, ${ }^{130}$ G. Petrucciani, ${ }^{130}$ A. Pfeiffer, ${ }^{130}$ M. Pierini, ${ }^{130}$ D. Rabady, ${ }^{130}$ A. Racz, ${ }^{130}$ M. Rieger, ${ }^{130}$ M. Rovere, ${ }^{130}$ H. Sakulin, ${ }^{130}$ J. Salfeld-Nebgen, ${ }^{130}$ S. Scarfi, ${ }^{130}$ C. Schäfer, ${ }^{130}$ C. Schwick, ${ }^{130}$ M. Selvaggi, ${ }^{130}$ A. Sharma, ${ }^{130}$ P. Silva, ${ }^{130}$ W. Snoeys, ${ }^{130}$ P. Sphicas, ${ }^{130, f f f}$ J. Steggemann, ${ }^{130}$ S. Summers, ${ }^{130}$ V. R. Tavolaro, ${ }^{130}$ D. Treille, ${ }^{130}$ A. Tsirou, ${ }^{130}$ G. P. Van Onsem, ${ }^{130}$ A. Vartak, ${ }^{130}$ M. Verzetti, ${ }^{130}$ K. A. Wozniak, ${ }^{130}$ W. D. Zeuner, ${ }^{130}$ L. Caminada, ${ }^{131, g g g}$ W. Erdmann, ${ }^{131}$ R. Horisberger, ${ }^{131}$ Q. Ingram, ${ }^{131}$ H. C. Kaestli, ${ }^{131}$ D. Kotlinski, ${ }^{131}$ U. Langenegger, ${ }^{131}$ T. Rohe, ${ }^{131}$ M. Backhaus, ${ }^{132}$ P. Berger, ${ }^{132}$ A. Calandri, ${ }^{132}$ N. Chernyavskaya, ${ }^{132}$ G. Dissertori, ${ }^{132}$ M. Dittmar, ${ }^{132}$ M. Donegà, ${ }^{132}$ C. Dorfer, ${ }^{132}$ T. Gadek, ${ }^{132}$ T. A. Gómez Espinosa, ${ }^{132}$ C. Grab, ${ }^{132}$ D. Hits, ${ }^{132}$ W. Lustermann, ${ }^{132}$ A.-M. Lyon, ${ }^{132}$ R. A. Manzoni, ${ }^{132}$ M. T. Meinhard, ${ }^{132}$ F. Micheli, ${ }^{132}$ F. Nessi-Tedaldi, ${ }^{132}$ F. Pauss, ${ }^{132}$ V. Perovic, ${ }^{132}$ G. Perrin, ${ }^{132}$ L. Perrozzi, ${ }^{132}$ S. Pigazzini, ${ }^{132}$ M. G. Ratti, ${ }^{132}$ M. Reichmann, ${ }^{132}$ C. Reissel, ${ }^{132}$ T. Reitenspiess, ${ }^{132}$ B. Ristic, ${ }^{132}$ D. Ruini, ${ }^{132}$ D. A. Sanz Becerra, ${ }^{132}$ M. Schönenberger, ${ }^{132}$ V. Stampf, ${ }^{132}$ M. L. Vesterbacka Olsson, ${ }^{132}$ R. Wallny, ${ }^{132}$

D. H. Zhu, ${ }^{132}$ C. Amsler, ${ }^{133, \text { hhh }}$ C. Botta, ${ }^{133}$ D. Brzhechko, ${ }^{133}$ M. F. Canelli, ${ }^{133}$ A. De Cosa, ${ }^{133}$ R. Del Burgo, ${ }^{133}$ J. K. Heikkilä, ${ }^{133}$ M. Huwiler, ${ }^{133}$ A. Jofrehei, ${ }^{133}$ B. Kilminster, ${ }^{133}$ S. Leontsinis, ${ }^{133}$ A. Macchiolo, ${ }^{133}$ P. Meiring, ${ }^{133}$ V. M. Mikuni, ${ }^{133}$ U. Molinatti, ${ }^{133}$ I. Neutelings, ${ }^{133}$ G. Rauco, ${ }^{133}$ A. Reimers, ${ }^{133}$ P. Robmann, ${ }^{133}$ K. Schweiger, ${ }^{133}$ Y. Takahashi, ${ }^{133}$ S. Wertz, ${ }^{133}$ C. Adloff, ${ }^{134, i i i}$ C. M. Kuo, ${ }^{134}$ W. Lin, ${ }^{134}$ A. Roy, ${ }^{134}$ T. Sarkar, ${ }^{134, j j}$ S. S. Yu, ${ }^{134}$ L. Ceard, ${ }^{135}$ P. Chang, ${ }^{135}$ Y. Chao, ${ }^{135}$ K. F. Chen, ${ }^{135}$ P. H. Chen, ${ }^{135}$ W.-S. Hou, ${ }^{135}$ Y. y. Li, ${ }^{135}$ R.-S. Lu, ${ }^{135}$ E. Paganis, ${ }^{135}$ A. Psallidas, ${ }^{135}$ A. Steen, ${ }^{135}$ E. Yazgan, ${ }^{135}$ B. Asavapibhop, ${ }^{136}$ C. Asawatangtrakuldee, ${ }^{136}$ N. Srimanobhas, ${ }^{136}$ F. Boran, ${ }^{137}$

S. Damarseckin, ${ }^{137, j j j}$ Z. S. Demiroglu, ${ }^{137}$ F. Dolek, ${ }^{137}$ C. Dozen, ${ }^{137, k k k}$ I. Dumanoglu, ${ }^{137,111}$ E. Eskut, ${ }^{137}$ G. Gokbulut, ${ }^{137}$ Y. Guler, ${ }^{137}$ E. Gurpinar Guler, ${ }^{137, \mathrm{mmm}}$ I. Hos, ${ }^{137, \mathrm{nnn}}$ C. Isik, ${ }^{137}$ E. E. Kangal, ${ }^{137,000}$ O. Kara, ${ }^{137}$ A. Kayis Topaksu, ${ }^{137}$ U. Kiminsu, ${ }^{137}$ G. Onengut, ${ }^{137}$ K. Ozdemir, ${ }^{137, p p p}$ A. Polatoz, ${ }^{137}$ A. E. Simsek, ${ }^{137}$ B. Tali, ${ }^{137, q q q}$ U. G. Tok, ${ }^{137}$

S. Turkcapar, ${ }^{137}$ I. S. Zorbakir, ${ }^{137}$ C. Zorbilmez, ${ }^{137}$ B. Isildak, ${ }^{138, \text { rrr }}$ G. Karapinar, ${ }^{138, \text { sss }}$ K. Ocalan, ${ }^{138, \text { ttt }}$ M. Yalvac, ${ }^{138, \text { uuu }}$ I. O. Atakisi, ${ }^{139}$ E. Gülmez, ${ }^{139}$ M. Kaya, ${ }^{139, \text { vvv }}$ O. Kaya ${ }^{139, \text { www }}$ Ö. Özçelik, ${ }^{139}$ S. Tekten, ${ }^{139, x x x}$ E. A. Yetkin, ${ }^{139, y y y}$ A. Cakir, ${ }^{140}$ K. Cankocak, ${ }^{140,111}$ Y. Komurcu, ${ }^{140}$ S. Sen, ${ }^{140, z z z}$ F. Aydogmus Sen, ${ }^{141}$ S. Cerci, ${ }^{141, q q q}$ B. Kaynak, ${ }^{141}$ 
S. Ozkorucuklu, ${ }^{141}$ D. Sunar Cerci, ${ }^{141, q q q}$ B. Grynyov, ${ }^{142}$ L. Levchuk, ${ }^{143}$ E. Bhal,${ }^{144}$ S. Bologna, ${ }^{144}$ J. J. Brooke, ${ }^{144}$ E. Clement, ${ }^{144}$ D. Cussans, ${ }^{144}$ H. Flacher, ${ }^{144}$ J. Goldstein, ${ }^{144}$ G. P. Heath,${ }^{144}$ H. F. Heath, ${ }^{144}$ L. Kreczko, ${ }^{144}$ B. Krikler, ${ }^{144}$ S. Paramesvaran, ${ }^{144}$ T. Sakuma, ${ }^{144}$ S. Seif El Nasr-Storey, ${ }^{144}$ V. J. Smith, ${ }^{144}$ J. Taylor, ${ }^{144}$ A. Titterton, ${ }^{144}$ K. W. Bell, ${ }^{145}$ A. Belyaev, ${ }^{145 \text {,aaa }}$ C. Brew, ${ }^{145}$ R. M. Brown, ${ }^{145}$ D. J. A. Cockerill, ${ }^{145}$ K. V. Ellis, ${ }^{145}$ K. Harder,${ }^{145}$ S. Harper,${ }^{145}$ J. Linacre, ${ }^{145}$ K. Manolopoulos, ${ }^{145}$ D. M. Newbold, ${ }^{145}$ E. Olaiya, ${ }^{145}$ D. Petyt, ${ }^{145}$ T. Reis, ${ }^{145}$ T. Schuh,${ }^{145}$

C. H. Shepherd-Themistocleous, ${ }^{145}$ A. Thea, ${ }^{145}$ I. R. Tomalin, ${ }^{145}$ T. Williams, ${ }^{145}$ R. Bainbridge, ${ }^{146}$ P. Bloch, ${ }^{146}$ S. Bonomally, ${ }^{146}$ J. Borg, ${ }^{146}$ S. Breeze, ${ }^{146}$ O. Buchmuller, ${ }^{146}$ A. Bundock, ${ }^{146}$ V. Cepaitis, ${ }^{146}$ G. S. Chahal, ${ }^{146, b b b b}$ D. Colling, ${ }^{146}$ P. Dauncey, ${ }^{146}$ G. Davies,${ }^{146}$ M. Della Negra, ${ }^{146}$ P. Everaerts,${ }^{146}$ G. Fedi, ${ }^{146}$ G. Hall, ${ }^{146}$ G. Iles, ${ }^{146}$ J. Langford, ${ }^{146}$ L. Lyons, ${ }^{146}$ A.-M. Magnan, ${ }^{146}$ S. Malik, ${ }^{146}$ A. Martelli, ${ }^{146}$ V. Milosevic, ${ }^{146}$ J. Nash, ${ }^{146, \text { cccc }}$ V. Palladino, ${ }^{146}$ M. Pesaresi, ${ }^{146}$ D. M. Raymond, ${ }^{146}$ A. Richards, ${ }^{146}$ A. Rose, ${ }^{146}$ E. Scott, ${ }^{146}$ C. Seez, ${ }^{146}$ A. Shtipliyski, ${ }^{146}$ M. Stoye, ${ }^{146}$ A. Tapper, ${ }^{146}$ K. Uchida, ${ }^{146}$ T. Virdee,${ }^{146, u}$ N. Wardle, ${ }^{146}$ S. N. Webb,${ }^{146}$ D. Winterbottom, ${ }^{146}$ A. G. Zecchinelli, ${ }^{146}$ J. E. Cole,${ }^{147}$ P. R. Hobson, ${ }^{147}$ A. Khan, ${ }^{147}$ P. Kyberd, ${ }^{147}$ C. K. Mackay, ${ }^{147}$ I. D. Reid, ${ }^{147}$ L. Teodorescu, ${ }^{147}$ S. Zahid, ${ }^{147}$ A. Brinkerhoff, ${ }^{148}$ K. Call, ${ }^{148}$ B. Caraway, ${ }^{148}$ J. Dittmann, ${ }^{148}$ K. Hatakeyama, ${ }^{148}$ A. R. Kanuganti, ${ }^{148}$ C. Madrid, ${ }^{148}$ B. McMaster, ${ }^{148}$ N. Pastika, ${ }^{148}$ S. Sawant, ${ }^{148}$ C. Smith, ${ }^{148}$ R. Bartek, ${ }^{149}$ A. Dominguez, ${ }^{149}$ R. Uniyal, ${ }^{149}$ A. M. Vargas Hernandez, ${ }^{149}$ A. Buccilli, ${ }^{150}$ O. Charaf, ${ }^{150}$ S. I. Cooper, ${ }^{150}$ S. V. Gleyzer ${ }^{150}$ C. Henderson, ${ }^{150}$ P. Rumerio, ${ }^{150}$ C. West, ${ }^{150}$ A. Akpinar, ${ }^{151}$ A. Albert, ${ }^{151}$ D. Arcaro, ${ }^{151}$ C. Cosby, ${ }^{151}$ Z. Demiragli, ${ }^{151}$ D. Gastler, ${ }^{151}$ C. Richardson, ${ }^{151}$ J. Rohlf, ${ }^{151}$ K. Salyer, ${ }^{151}$ D. Sperka, ${ }^{151}$ D. Spitzbart, ${ }^{151}$ I. Suarez, ${ }^{151}$ S. Yuan, ${ }^{151}$ D. Zou, ${ }^{151}$ G. Benelli, ${ }^{152}$ B. Burkle,,${ }^{152}$ X. Coubez, ${ }^{152, v}$ D. Cutts, ${ }^{152}$ Y. t. Duh, ${ }^{152}$ M. Hadley, ${ }^{152}$ U. Heintz, ${ }^{152}$ J. M. Hogan, ${ }^{152, \text { dddd }}$ K. H. M. Kwok, ${ }^{152}$ E. Laird, ${ }^{152}$ G. Landsberg, ${ }^{152}$ K. T. Lau, ${ }^{152}$ J. Lee, ${ }^{152}$ M. Narain, ${ }^{152}$ S. Sagir, ${ }^{152, \text { eeee }}$ R. Syarif, ${ }^{152}$ E. Usai, ${ }^{152}$ W. Y. Wong,${ }^{152}$ D. Yu, ${ }^{152}$ W. Zhang, ${ }^{152}$ R. Band, ${ }^{153}$ C. Brainerd, ${ }^{153}$ R. Breedon, ${ }^{153}$ M. Calderon De La Barca Sanchez,${ }^{153}$ M. Chertok, ${ }^{153}$ J. Conway, ${ }^{153}$ R. Conway, ${ }^{153}$ P. T. Cox,${ }^{153}$ R. Erbacher, ${ }^{153}$ C. Flores, ${ }^{153}$ G. Funk, ${ }^{153}$ F. Jensen,${ }^{153}$ W. Ko, ${ }^{153, a}$ O. Kukral, ${ }^{153}$ R. Lander, ${ }^{153}$ M. Mulhearn, ${ }^{153}$ D. Pellett, ${ }^{153}$ J. Pilot, ${ }^{153}$ M. Shi, ${ }^{153}$ D. Taylor, ${ }^{153}$ K. Tos, ${ }^{153}$ M. Tripathi, ${ }^{153}$ Y. Yao, ${ }^{153}$ F. Zhang, ${ }^{153}$ M. Bachtis, ${ }^{154}$ R. Cousins, ${ }^{154}$ A. Dasgupta, ${ }^{154}$ A. Florent, ${ }^{154}$ D. Hamilton, ${ }^{154}$ J. Hauser, ${ }^{154}$ M. Ignatenko, ${ }^{154}$ T. Lam, ${ }^{154}$ N. Mccoll, ${ }^{154}$ W. A. Nash, ${ }^{154}$ S. Regnard, ${ }^{154}$ D. Saltzberg, ${ }^{154}$ C. Schnaible, ${ }^{154}$ B. Stone, ${ }^{154}$ V. Valuev, ${ }^{154}$ K. Burt, ${ }^{155}$ Y. Chen, ${ }^{155}$ R. Clare, ${ }^{155}$ J. W. Gary, ${ }^{155}$ S. M. A. Ghiasi Shirazi, ${ }^{155}$ G. Hanson, ${ }^{155}$ G. Karapostoli, ${ }^{155}$ O. R. Long, ${ }^{155}$ N. Manganelli, ${ }^{155}$ M. Olmedo Negrete, ${ }^{155}$ M. I. Paneva, ${ }^{155}$ W. Si, ${ }^{155}$ S. Wimpenny, ${ }^{155}$ Y. Zhang, ${ }^{155}$ J. G. Branson, ${ }^{156}$ P. Chang, ${ }^{156}$ S. Cittolin, ${ }^{156}$ S. Cooperstein, ${ }^{156}$ N. Deelen, ${ }^{156}$ M. Derdzinski, ${ }^{156}$ J. Duarte, ${ }^{156}$ R. Gerosa ${ }^{156}$ D. Gilbert, ${ }^{156}$ B. Hashemi, ${ }^{156}$ V. Krutelyov, ${ }^{156}$ J. Letts, ${ }^{156}$ M. Masciovecchio, ${ }^{156}$ S. May, ${ }^{156}$ S. Padhi, ${ }^{156}$ M. Pieri, ${ }^{156}$ V. Sharma, ${ }^{156}$ M. Tadel,,${ }^{156}$ F. Würthwein, ${ }^{156}$ A. Yagil, ${ }^{156}$ N. Amin, ${ }^{157}$ C. Campagnari, ${ }^{157}$ M. Citron, ${ }^{157}$ A. Dorsett, ${ }^{157}$ V. Dutta, ${ }^{157}$ J. Incandela, ${ }^{157}$ B. Marsh, ${ }^{157}$ H. Mei, ${ }^{157}$ A. Ovcharova, ${ }^{157}$ H. Qu ${ }^{157}$ M. Quinnan, ${ }^{157}$ J. Richman, ${ }^{157}$ U. Sarica, ${ }^{157}$ D. Stuart, ${ }^{157}$

S. Wang, ${ }^{157}$ D. Anderson, ${ }^{158}$ A. Bornheim, ${ }^{158}$ O. Cerri, ${ }^{158}$ I. Dutta, ${ }^{158}$ J. M. Lawhorn, ${ }^{158}$ N. Lu, ${ }^{158}$ J. Mao, ${ }^{158}$

H. B. Newman, ${ }^{158}$ T. Q. Nguyen, ${ }^{158}$ J. Pata, ${ }^{158}$ M. Spiropulu, ${ }^{158}$ J. R. Vlimant, ${ }^{158}$ S. Xie, ${ }^{158}$ Z. Zhang, ${ }^{158}$ R. Y. Zhu, ${ }^{158}$ J. Alison, ${ }^{159}$ M. B. Andrews, ${ }^{159}$ T. Ferguson, ${ }^{159}$ T. Mudholkar, ${ }^{159}$ M. Paulini, ${ }^{159}$ M. Sun ${ }^{159}$ I. Vorobiev, ${ }^{159}$ J. P. Cumalat, ${ }^{160}$ W. T. Ford, ${ }^{160}$ E. MacDonald, ${ }^{160}$ T. Mulholland, ${ }^{160}$ R. Patel, ${ }^{160}$ A. Perloff, ${ }^{160}$ K. Stenson, ${ }^{160}$ K. A. Ulmer, ${ }^{160}$ S. R. Wagner, ${ }^{160}$ J. Alexander, ${ }^{161}$ Y. Cheng, ${ }^{161}$ J. Chu, ${ }^{161}$ D. J. Cranshaw, ${ }^{161}$ A. Datta ${ }^{161}$ A. Frankenthal, ${ }^{161}$ K. Mcdermott, ${ }^{161}$ J. Monroy, ${ }^{161}$ J. R. Patterson, ${ }^{161}$ D. Quach,${ }^{161}$ A. Ryd, ${ }^{161}$ W. Sun, ${ }^{161}$ S. M. Tan, ${ }^{161}$ Z. Tao,${ }^{161}$ J. Thom, ${ }^{161}$ P. Wittich, ${ }^{161}$ M. Zientek, ${ }^{161}$ S. Abdullin, ${ }^{162}$ M. Albrow, ${ }^{162}$ M. Alyari, ${ }^{162}$ G. Apollinari, ${ }^{162}$ A. Apresyan, ${ }^{162}$ A. Apyan, ${ }^{162}$ S. Banerjee, ${ }^{162}$

L. A. T. Bauerdick, ${ }^{162}$ A. Beretvas, ${ }^{162}$ D. Berry, ${ }^{162}$ J. Berryhill, ${ }^{162}$ P. C. Bhat, ${ }^{162}$ K. Burkett, ${ }^{162}$ J. N. Butler, ${ }^{162}$ A. Canepa, ${ }^{162}$ G. B. Cerati, ${ }^{162}$ H. W. K. Cheung, ${ }^{162}$ F. Chlebana, ${ }^{162}$ M. Cremonesi, ${ }^{162}$ V. D. Elvira, ${ }^{162}$ J. Freeman, ${ }^{162}$ Z. Gecse, ${ }^{162}$ E. Gottschalk, ${ }^{162}$ L. Gray, ${ }^{162}$ D. Green, ${ }^{162}$ S. Grünendahl, ${ }^{162}$ O. Gutsche, ${ }^{162}$ R. M. Harris, ${ }^{162}$ S. Hasegawa, ${ }^{162}$ R. Heller, ${ }^{162}$ T. C. Herwig, ${ }^{162}$ J. Hirschauer, ${ }^{162}$ B. Jayatilaka, ${ }^{162}$ S. Jindariani, ${ }^{162}$ M. Johnson, ${ }^{162}$ U. Joshi, ${ }^{162}$ P. Klabbers, ${ }^{162}$ T. Klijnsma, ${ }^{162}$ B. Klima, ${ }^{162}$ M. J. Kortelainen, ${ }^{162}$ S. Lammel, ${ }^{162}$ D. Lincoln, ${ }^{162}$ R. Lipton, ${ }^{162}$ M. Liu, ${ }^{162}$ T. Liu, ${ }^{162}$ J. Lykken, ${ }^{162}$ K. Maeshima, ${ }^{162}$ D. Mason, ${ }^{162}$ P. McBride, ${ }^{162}$ P. Merkel, ${ }^{162}$ S. Mrenna, ${ }^{162}$ S. Nahn, ${ }^{162}$ V. O'Dell, ${ }^{162}$ V. Papadimitriou, ${ }^{162}$ K. Pedro, ${ }^{162}$ C. Pena, ${ }^{162, \text { aaa }}$ O. Prokofyev, ${ }^{162}$ F. Ravera, ${ }^{162}$ A. Reinsvold Hall, ${ }^{162}$ L. Ristori, ${ }^{162}$ B. Schneider, ${ }^{162}$ E. Sexton-Kennedy, ${ }^{162}$ N. Smith, ${ }^{162}$ A. Soha, ${ }^{162}$ W. J. Spalding, ${ }^{162}$ L. Spiegel, ${ }^{162}$ S. Stoynev, ${ }^{162}$ J. Strait, ${ }^{162}$ L. Taylor, ${ }^{162}$ S. Tkaczyk, ${ }^{162}$ N. V. Tran, ${ }^{162}$ L. Uplegger, ${ }^{162}$ E. W. Vaandering, ${ }^{162}$ H. A. Weber, ${ }^{162}$ A. Woodard, ${ }^{162}$ D. Acosta, ${ }^{163}$ P. Avery, ${ }^{163}$ D. Bourilkov, ${ }^{163}$ L. Cadamuro, ${ }^{163}$ V. Cherepanov, ${ }^{163}$ F. Errico, ${ }^{163}$ R. D. Field ${ }^{163}$ D. Guerrero, ${ }^{163}$ B. M. Joshi, ${ }^{163}$ M. Kim, ${ }^{163}$ J. Konigsberg, ${ }^{163}$ A. Korytov, ${ }^{163}$ K. H. Lo, ${ }^{163}$ K. Matchev, ${ }^{163}$ N. Menendez, ${ }^{163}$ G. Mitselmakher, ${ }^{163}$ D. Rosenzweig, ${ }^{163}$ K. Shi, ${ }^{163}$ J. Wang, ${ }^{163}$ S. Wang, ${ }^{163}$ X. Zuo, ${ }^{163}$ T. Adams, ${ }^{164}$ A. Askew, ${ }^{164}$ D. Diaz, ${ }^{164}$ 
R. Habibullah, ${ }^{164}$ S. Hagopian, ${ }^{164}$ V. Hagopian, ${ }^{164}$ K. F. Johnson, ${ }^{164}$ R. Khurana, ${ }^{164}$ T. Kolberg, ${ }^{164}$ G. Martinez, ${ }^{164}$ H. Prosper, ${ }^{164}$ C. Schiber, ${ }^{164}$ R. Yohay, ${ }^{164}$ J. Zhang, ${ }^{164}$ M. M. Baarmand, ${ }^{165}$ S. Butalla, ${ }^{165}$ T. Elkafrawy, ${ }^{165, m}$ M. Hohlmann, ${ }^{165}$ D. Noonan, ${ }^{165}$ M. Rahmani, ${ }^{165}$ M. Saunders, ${ }^{165}$ F. Yumiceva, ${ }^{165}$ M. R. Adams, ${ }^{166}$ L. Apanasevich, ${ }^{166}$ H. Becerril Gonzalez, ${ }^{166}$ R. Cavanaugh, ${ }^{166}$ X. Chen, ${ }^{166}$ S. Dittmer, ${ }^{166}$ O. Evdokimov, ${ }^{166}$ C. E. Gerber, ${ }^{166}$ D. A. Hangal, ${ }^{166}$ D. J. Hofman, ${ }^{166}$ C. Mills, ${ }^{166}$ G. Oh, ${ }^{166}$ T. Roy, ${ }^{166}$ M. B. Tonjes, ${ }^{166}$ N. Varelas, ${ }^{166}$ J. Viinikainen, ${ }^{166}$ X. Wang, ${ }^{166}$ Z. Wu, ${ }^{166}$ M. Alhusseini, ${ }^{167}$ K. Dilsiz, ${ }^{167, f f f f}$ S. Durgut, ${ }^{167}$ R. P. Gandrajula, ${ }^{167}$ M. Haytmyradov, ${ }^{167}$ V. Khristenko, ${ }^{167}$ O. K. Köseyan, ${ }^{167}$ J.-P. Merlo, ${ }^{167}$ A. Mestvirishvili, ${ }^{167, g g g g}$ A. Moeller, ${ }^{167}$ J. Nachtman, ${ }^{167}$ H. Ogul, ${ }^{167, \text { hhhh }}$ Y. Onel, ${ }^{167}$ F. Ozok, ${ }^{167, \text { iiii }}$ A. Penzo, ${ }^{167}$ C. Snyder, ${ }^{167}$ E. Tiras, ${ }^{167}$ J. Wetzel, ${ }^{167}$ K. Yi, ${ }^{167, j j j}$ O. Amram, ${ }^{168}$ B. Blumenfeld, ${ }^{168}$ L. Corcodilos, ${ }^{168}$ M. Eminizer, ${ }^{168}$ A. V. Gritsan, ${ }^{168}$ S. Kyriacou, ${ }^{168}$ P. Maksimovic, ${ }^{168}$ C. Mantilla, ${ }^{168}$ J. Roskes, ${ }^{168}$ M. Swartz, ${ }^{168}$ T. Á. Vámi, ${ }^{168}$ C. Baldenegro Barrera, ${ }^{169}$ P. Baringer, ${ }^{169}$ A. Bean, ${ }^{169}$ A. Bylinkin, ${ }^{169}$ T. Isidori, ${ }^{169}$ S. Khalil, ${ }^{169}$ J. King, ${ }^{169}$ G. Krintiras, ${ }^{169}$ A. Kropivnitskaya, ${ }^{169}$ C. Lindsey, ${ }^{169}$ N. Minafra, ${ }^{169}$ M. Murray, ${ }^{169}$ C. Rogan, ${ }^{169}$ C. Royon, ${ }^{169}$ S. Sanders, ${ }^{169}$ E. Schmitz, ${ }^{169}$ J. D. Tapia Takaki, ${ }^{169}$ Q. Wang, ${ }^{169}$ J. Williams, ${ }^{169}$ G. Wilson, ${ }^{169}$ S. Duric, ${ }^{170}$ A. Ivanov, ${ }^{170}$ K. Kaadze, ${ }^{170}$ D. Kim, ${ }^{170}$ Y. Maravin, ${ }^{170}$ T. Mitchell, ${ }^{170}$ A. Modak, ${ }^{170}$ A. Mohammadi, ${ }^{170}$ F. Rebassoo, ${ }^{171}$ D. Wright, ${ }^{171}$ E. Adams, ${ }^{172}$

A. Baden, ${ }^{172}$ O. Baron, ${ }^{172}$ A. Belloni, ${ }^{172}$ S. C. Eno, ${ }^{172}$ Y. Feng, ${ }^{172}$ N. J. Hadley, ${ }^{172}$ S. Jabeen, ${ }^{172}$ G. Y. Jeng, ${ }^{172}$ R. G. Kellogg, ${ }^{172}$ T. Koeth, ${ }^{172}$ A. C. Mignerey, ${ }^{172}$ S. Nabili, ${ }^{172}$ M. Seidel, ${ }^{172}$ A. Skuja, ${ }^{172}$ S. C. Tonwar, ${ }^{172}$ L. Wang, ${ }^{172}$ K. Wong, ${ }^{172}$ D. Abercrombie, ${ }^{173}$ B. Allen, ${ }^{173}$ R. Bi, ${ }^{173}$ S. Brandt, ${ }^{173}$ W. Busza, ${ }^{173}$ I. A. Cali, ${ }^{173}$ Y. Chen, ${ }^{173}$ M. D'Alfonso, ${ }^{173}$ G. Gomez Ceballos, ${ }^{173}$ M. Goncharov, ${ }^{173}$ P. Harris, ${ }^{173}$ D. Hsu, ${ }^{173}$ M. Hu, ${ }^{173}$ M. Klute, ${ }^{173}$ D. Kovalskyi, ${ }^{173}$ J. Krupa, ${ }^{173}$ Y.-J. Lee, ${ }^{173}$ P. D. Luckey, ${ }^{173}$ B. Maier, ${ }^{173}$ A. C. Marini, ${ }^{173}$ C. Mcginn, ${ }^{173}$ C. Mironov, ${ }^{173}$ S. Narayanan, ${ }^{173}$ X. Niu, ${ }^{173}$

C. Paus, ${ }^{173}$ D. Rankin, ${ }^{173}$ C. Roland, ${ }^{173}$ G. Roland, ${ }^{173}$ Z. Shi, ${ }^{173}$ G. S. F. Stephans, ${ }^{173}$ K. Sumorok, ${ }^{173}$ K. Tatar, ${ }^{173}$ D. Velicanu, ${ }^{173}$ J. Wang, ${ }^{173}$ T. W. Wang, ${ }^{173}$ Z. Wang, ${ }^{173}$ B. Wyslouch, ${ }^{173}$ R. M. Chatterjee, ${ }^{174}$ A. Evans, ${ }^{174}$ S. Guts, ${ }^{174, a}$ P. Hansen, ${ }^{174}$ J. Hiltbrand, ${ }^{174}$ Sh. Jain, ${ }^{174}$ M. Krohn, ${ }^{174}$ Y. Kubota, ${ }^{174}$ Z. Lesko, ${ }^{174}$ J. Mans, ${ }^{174}$ M. Revering, ${ }^{174}$ R. Rusack, ${ }^{174}$ R. Saradhy, ${ }^{174}$ N. Schroeder, ${ }^{174}$ N. Strobbe, ${ }^{174}$ M. A. Wadud, ${ }^{174}$ J. G. Acosta, ${ }^{175}$ S. Oliveros, ${ }^{175}$ K. Bloom, ${ }^{176}$ S. Chauhan, ${ }^{176}$ D. R. Claes, ${ }^{176}$ C. Fangmeier, ${ }^{176}$ L. Finco, ${ }^{176}$ F. Golf, ${ }^{176}$ J. R. González Fernández, ${ }^{176}$ I. Kravchenko, ${ }^{176}$ J. E. Siado, ${ }^{176}$ G. R. Snow, ${ }^{176, a}$ B. Stieger, ${ }^{176}$ W. Tabb, ${ }^{176}$ F. Yan, ${ }^{176}$ G. Agarwal, ${ }^{177}$ C. Harrington, ${ }^{177}$ L. Hay, ${ }^{177}$ I. Iashvili, ${ }^{177}$ A. Kharchilava, ${ }^{177}$ C. McLean, ${ }^{177}$ D. Nguyen, ${ }^{177}$ A. Parker, ${ }^{177}$ J. Pekkanen, ${ }^{177}$ S. Rappoccio, ${ }^{177}$ B. Roozbahani, ${ }^{177}$ G. Alverson, ${ }^{178}$ E. Barberis, ${ }^{178}$ C. Freer, ${ }^{178}$ Y. Haddad, ${ }^{178}$ A. Hortiangtham, ${ }^{178}$ G. Madigan, ${ }^{178}$ B. Marzocchi, ${ }^{178}$ D. M. Morse, ${ }^{178}$ V. Nguyen, ${ }^{178}$ T. Orimoto, ${ }^{178}$ L. Skinnari, ${ }^{178}$ A. Tishelman-Charny, ${ }^{178}$ T. Wamorkar, ${ }^{178}$ B. Wang, ${ }^{178}$ A. Wisecarver, ${ }^{178}$ D. Wood, ${ }^{178}$ S. Bhattacharya, ${ }^{179}$ J. Bueghly, ${ }^{179}$ Z. Chen, ${ }^{179}$ A. Gilbert, ${ }^{179}$ T. Gunter, ${ }^{179}$ K. A. Hahn, ${ }^{179}$ N. Odell, ${ }^{179}$ M. H. Schmitt, ${ }^{179}$ K. Sung, ${ }^{179}$ M. Velasco, ${ }^{179}$ R. Bucci, ${ }^{180}$ N. Dev, ${ }^{180}$ R. Goldouzian, ${ }^{180}$ M. Hildreth, ${ }^{180}$ K. Hurtado Anampa, ${ }^{180}$ C. Jessop, ${ }^{180}$ D. J. Karmgard, ${ }^{180}$ K. Lannon, ${ }^{180}$ W. Li, ${ }^{180}$ N. Loukas, ${ }^{180}$ N. Marinelli, ${ }^{180}$ I. Mcalister, ${ }^{180}$ F. Meng, ${ }^{180}$ K. Mohrman, ${ }^{180}$ Y. Musienko, ${ }^{180, t t}$ R. Ruchti, ${ }^{180}$ P. Siddireddy, ${ }^{180}$ S. Taroni, ${ }^{180}$ M. Wayne, ${ }^{180}$ A. Wightman, ${ }^{180}$ M. Wolf, ${ }^{180}$ L. Zygala, ${ }^{180}$ J. Alimena, ${ }^{181}$ B. Bylsma, ${ }^{181}$ B. Cardwell, ${ }^{181}$ L. S. Durkin, ${ }^{181}$ B. Francis, ${ }^{181}$ C. Hill, ${ }^{181}$ A. Lefeld, ${ }^{181}$ B. L. Winer, ${ }^{181}$ B. R. Yates, ${ }^{181}$ G. Dezoort, ${ }^{182}$ P. Elmer, ${ }^{182}$ B. Greenberg, ${ }^{182}$ N. Haubrich, ${ }^{182}$ S. Higginbotham, ${ }^{182}$ A. Kalogeropoulos, ${ }^{182}$ G. Kopp, ${ }^{182}$ S. Kwan, ${ }^{182}$ D. Lange, ${ }^{182}$ M. T. Lucchini, ${ }^{182}$ J. Luo, ${ }^{182}$ D. Marlow, ${ }^{182}$ K. Mei, ${ }^{182}$ I. Ojalvo, ${ }^{182}$ J. Olsen, ${ }^{182}$ C. Palmer, ${ }^{182}$ P. Piroué, ${ }^{182}$ D. Stickland, ${ }^{182}$ C. Tully, ${ }^{182}$ S. Malik, ${ }^{183}$ S. Norberg, ${ }^{183}$ V. E. Barnes, ${ }^{184}$ R. Chawla, ${ }^{184}$ S. Das, ${ }^{184}$ L. Gutay, ${ }^{184}$ M. Jones, ${ }^{184}$ A. W. Jung, ${ }^{184}$ B. Mahakud, ${ }^{184}$ G. Negro, ${ }^{184}$ N. Neumeister, ${ }^{184}$ C. C. Peng, ${ }^{184}$ S. Piperov, ${ }^{184}$ H. Qiu, ${ }^{184}$ J. F. Schulte, ${ }^{184}$ N. Trevisani, ${ }^{184}$ F. Wang, ${ }^{184}$ R. Xiao, ${ }^{184}$ W. Xie, ${ }^{184}$ T. Cheng, ${ }^{185}$ J. Dolen, ${ }^{185}$ N. Parashar, ${ }^{185}$ M. Stojanovic, ${ }^{185}$ A. Baty, ${ }^{186}$ S. Dildick, ${ }^{186}$ K. M. Ecklund, ${ }^{186}$ S. Freed, ${ }^{186}$ F. J. M. Geurts, ${ }^{186}$ M. Kilpatrick, ${ }^{186}$ A. Kumar, ${ }^{186}$ W. Li, ${ }^{186}$ B. P. Padley, ${ }^{186}$ R. Redjimi, ${ }^{186}$ J. Roberts, ${ }^{186, a}$ J. Rorie, ${ }^{186}$ W. Shi, ${ }^{186}$ A. G. Stahl Leiton, ${ }^{186}$ A. Bodek, ${ }^{187}$ P. de Barbaro, ${ }^{187}$ R. Demina, ${ }^{187}$ J. L. Dulemba, ${ }^{187}$ C. Fallon, ${ }^{187}$ T. Ferbel, ${ }^{187}$ M. Galanti, ${ }^{187}$ A. Garcia-Bellido, ${ }^{187}$ O. Hindrichs, ${ }^{187}$ A. Khukhunaishvili, ${ }^{187}$ E. Ranken, ${ }^{187}$ R. Taus, ${ }^{187}$ B. Chiarito, ${ }^{188}$ J. P. Chou, ${ }^{188}$ A. Gandrakota, ${ }^{188}$ Y. Gershtein, ${ }^{188}$ E. Halkiadakis, ${ }^{188}$ A. Hart, ${ }^{188}$ M. Heindl, ${ }^{188}$ E. Hughes, ${ }^{188}$ S. Kaplan, ${ }^{188}$ O. Karacheban, ${ }^{188, y}$ I. Laflotte, ${ }^{188}$ A. Lath, ${ }^{188}$ R. Montalvo, ${ }^{188}$ K. Nash, ${ }^{188}$ M. Osherson, ${ }^{188}$ S. Salur, ${ }^{188}$ S. Schnetzer, ${ }^{188}$ S. Somalwar, ${ }^{188}$ R. Stone, ${ }^{188}$ S. A. Thayil, ${ }^{188}$ S. Thomas, ${ }^{188}$ H. Wang, ${ }^{188}$ H. Acharya, ${ }^{189}$ A. G. Delannoy, ${ }^{189}$ S. Spanier, ${ }^{189}$ O. Bouhali, ${ }^{190, k k k}$ M. Dalchenko, ${ }^{190}$ A. Delgado, ${ }^{190}$ R. Eusebi, ${ }^{190}$ J. Gilmore, ${ }^{190}$ T. Huang, ${ }^{190}$ T. Kamon, ${ }^{190,1111}$ H. Kim, ${ }^{190}$ S. Luo, ${ }^{190}$ S. Malhotra, ${ }^{190}$ R. Mueller, ${ }^{190}$ D. Overton, ${ }^{190}$ L. Perniè, ${ }^{190}$ D. Rathjens, ${ }^{190}$ A. Safonov, ${ }^{190}$ J. Sturdy, ${ }^{190}$ N. Akchurin, ${ }^{191}$ J. Damgov, ${ }^{191}$ V. Hegde, ${ }^{191}$ S. Kunori, ${ }^{191}$ K. Lamichhane, ${ }^{191}$ S. W. Lee, ${ }^{191}$ T. Mengke, ${ }^{191}$ S. Muthumuni, ${ }^{191}$ T. Peltola, ${ }^{191}$ S. Undleeb, ${ }^{191}$ I. Volobouev, ${ }^{191}$ Z. Wang, ${ }^{191}$ A. Whitbeck, ${ }^{191}$ E. Appelt, ${ }^{192}$ S. Greene, ${ }^{192}$ A. Gurrola, ${ }^{192}$ R. Janjam, ${ }^{192}$ W. Johns, ${ }^{192}$ C. Maguire, ${ }^{192}$ A. Melo, ${ }^{192}$ H. Ni, ${ }^{192}$ K. Padeken, ${ }^{192}$ F. Romeo, ${ }^{192}$ P. Sheldon, ${ }^{192}$ S. Tuo, ${ }^{192}$ 
J. Velkovska, ${ }^{192}$ M. Verweij, ${ }^{192}$ L. Ang, ${ }^{193}$ M. W. Arenton, ${ }^{193}$ B. Cox, ${ }^{193}$ G. Cummings,${ }^{193}$ J. Hakala, ${ }^{193}$ R. Hirosky, ${ }^{193}$ M. Joyce, ${ }^{193}$ A. Ledovskoy, ${ }^{193}$ C. Neu, ${ }^{193}$ B. Tannenwald, ${ }^{193}$ Y. Wang, ${ }^{193}$ E. Wolfe, ${ }^{193}$ F. Xia, ${ }^{193}$ P. E. Karchin, ${ }^{194}$ N. Poudyal, ${ }^{194}$ P. Thapa,${ }^{194}$ K. Black, ${ }^{195}$ T. Bose, ${ }^{195}$ J. Buchanan, ${ }^{195}$ C. Caillol, ${ }^{195}$ S. Dasu, ${ }^{195}$ I. De Bruyn, ${ }^{195}$ C. Galloni, ${ }^{195}$ H. He,${ }^{195}$ M. Herndon, ${ }^{195}$ A. Hervé, ${ }^{195}$ U. Hussain, ${ }^{195}$ A. Lanaro, ${ }^{195}$ A. Loeliger, ${ }^{195}$ R. Loveless, ${ }^{195}$

J. Madhusudanan Sreekala, ${ }^{195}$ A. Mallampalli, ${ }^{195}$ D. Pinna, ${ }^{195}$ T. Ruggles,${ }^{195}$ A. Savin, ${ }^{195}$ V. Shang, ${ }^{195}$ V. Sharma, ${ }^{195}$ W. H. Smith, ${ }^{195}$ D. Teague, ${ }^{195}$ S. Trembath-Reichert, ${ }^{195}$ and W. Vetens ${ }^{195}$

\title{
CMS Collaboration
}

\author{
${ }^{1}$ Yerevan Physics Institute, Yerevan, Armenia \\ ${ }^{2}$ Institut für Hochenergiephysik, Wien, Austria \\ ${ }^{3}$ Institute for Nuclear Problems, Minsk, Belarus \\ ${ }^{4}$ Universiteit Antwerpen, Antwerpen, Belgium \\ ${ }^{5}$ Vrije Universiteit Brussel, Brussel, Belgium \\ ${ }^{6}$ Université Libre de Bruxelles, Bruxelles, Belgium \\ ${ }^{7}$ Ghent University, Ghent, Belgium \\ ${ }^{8}$ Université Catholique de Louvain, Louvain-la-Neuve, Belgium \\ ${ }^{9}$ Centro Brasileiro de Pesquisas Fisicas, Rio de Janeiro, Brazil \\ ${ }^{10}$ Universidade do Estado do Rio de Janeiro, Rio de Janeiro, Brazil \\ ${ }^{11 a}$ Universidade Estadual Paulista, São Paulo, Brazil \\ ${ }^{11 \mathrm{~b}}$ Universidade Federal do ABC, São Paulo, Brazil \\ ${ }^{12}$ Institute for Nuclear Research and Nuclear Energy, Bulgarian Academy of Sciences, Sofia, Bulgaria \\ ${ }^{13}$ University of Sofia, Sofia, Bulgaria \\ ${ }^{14}$ Beihang University, Beijing, China \\ ${ }^{15}$ Department of Physics, Tsinghua University, Beijing, China \\ ${ }^{16}$ Institute of High Energy Physics, Beijing, China \\ ${ }^{17}$ State Key Laboratory of Nuclear Physics and Technology, Peking University, Beijing, China \\ ${ }^{18}$ Sun Yat-Sen University, Guangzhou, China \\ ${ }^{19}$ Institute of Modern Physics and Key Laboratory of Nuclear Physics and Ion-beam Application \\ (MOE)_Fudan University, Shanghai, China \\ ${ }^{20}$ Zhejiang University, Hangzhou, China \\ ${ }^{21}$ Universidad de Los Andes, Bogota, Colombia \\ ${ }^{22}$ Universidad de Antioquia, Medellin, Colombia \\ ${ }^{23}$ University of Split, Faculty of Electrical Engineering, Mechanical Engineering and Naval Architecture, \\ Split, Croatia \\ ${ }^{24}$ University of Split, Faculty of Science, Split, Croatia \\ ${ }^{25}$ Institute Rudjer Boskovic, Zagreb, Croatia \\ ${ }^{26}$ University of Cyprus, Nicosia, Cyprus \\ ${ }^{27}$ Charles University, Prague, Czech Republic \\ ${ }^{28}$ Escuela Politecnica Nacional, Quito, Ecuador \\ ${ }^{29}$ Universidad San Francisco de Quito, Quito, Ecuador \\ ${ }^{30}$ Academy of Scientific Research and Technology of the Arab Republic of Egypt, Egyptian Network of \\ High Energy Physics, Cairo, Egypt \\ ${ }^{31}$ Center for High Energy Physics (CHEP-FU), Fayoum University, El-Fayoum, Egypt \\ ${ }^{32}$ National Institute of Chemical Physics and Biophysics, Tallinn, Estonia \\ ${ }^{33}$ Department of Physics, University of Helsinki, Helsinki, Finland \\ ${ }^{34}$ Helsinki Institute of Physics, Helsinki, Finland \\ ${ }^{35}$ Lappeenranta University of Technology, Lappeenranta, Finland \\ ${ }^{36}$ IRFU, CEA, Université Paris-Saclay, Gif-sur-Yvette, France \\ ${ }^{37}$ Laboratoire Leprince-Ringuet, CNRS/IN2P3, Ecole Polytechnique, Institut Polytechnique de Paris, \\ Palaiseau, France \\ ${ }^{38}$ Université de Strasbourg, CNRS, IPHC UMR 7178, Strasbourg, France \\ ${ }^{39}$ Université de Lyon, Université Claude Bernard Lyon 1, CNRS-IN2P3, Institut de Physique Nucléaire de \\ Lyon, Villeurbanne, France \\ ${ }^{40}$ Georgian Technical University, Tbilisi, Georgia \\ ${ }^{41}$ RWTH Aachen University, I. Physikalisches Institut, Aachen, Germany \\ ${ }^{42}$ RWTH Aachen University, III. Physikalisches Institut A, Aachen, Germany \\ ${ }^{43}$ RWTH Aachen University, III. Physikalisches Institut B, Aachen, Germany
}


${ }^{44}$ Deutsches Elektronen-Synchrotron, Hamburg, Germany

${ }^{45}$ University of Hamburg, Hamburg, Germany

${ }^{46}$ Karlsruher Institut fuer Technologie, Karlsruhe, Germany

${ }^{47}$ Institute of Nuclear and Particle Physics (INPP), NCSR Demokritos, Aghia Paraskevi, Greece

${ }^{48}$ National and Kapodistrian University of Athens, Athens, Greece

${ }^{49}$ National Technical University of Athens, Athens, Greece

${ }^{50}$ University of Ioánnina, Ioánnina, Greece

${ }^{51}$ MTA-ELTE Lendület CMS Particle and Nuclear Physics Group, Eötvös Loránd University, Budapest, Hungary

${ }^{52}$ Wigner Research Centre for Physics, Budapest, Hungary

${ }^{53}$ Institute of Nuclear Research ATOMKI, Debrecen, Hungary

${ }^{54}$ Institute of Physics, University of Debrecen, Debrecen, Hungary

${ }^{55}$ Eszterhazy Karoly University, Karoly Robert Campus, Gyongyos, Hungary

${ }^{56}$ Indian Institute of Science (IISc), Bangalore, India

${ }^{57}$ National Institute of Science Education and Research, HBNI, Bhubaneswar, India

${ }^{58}$ Panjab University, Chandigarh, India

${ }^{59}$ University of Delhi, Delhi, India

${ }^{60}$ Saha Institute of Nuclear Physics, HBNI, Kolkata, India

${ }^{61}$ Indian Institute of Technology Madras, Madras, India

${ }^{62}$ Bhabha Atomic Research Centre, Mumbai, India

${ }^{63}$ Tata Institute of Fundamental Research-A, Mumbai, India

${ }^{64}$ Tata Institute of Fundamental Research-B, Mumbai, India

${ }^{65}$ Indian Institute of Science Education and Research (IISER), Pune, India

${ }^{66}$ Department of Physics, Isfahan University of Technology, Isfahan, Iran

${ }^{67}$ Institute for Research in Fundamental Sciences (IPM), Tehran, Iran

${ }^{68}$ University College Dublin, Dublin, Ireland

${ }^{69 a}$ INFN Sezione di Bari, Bari, Italy

${ }^{69 \mathrm{~b}}$ Università di Bari, Bari, Italy

${ }^{69 \mathrm{c}}$ Politecnico di Bari, Bari, Italy

${ }^{70 a}$ INFN Sezione di Bologna, Bologna, Italy

${ }^{70 \mathrm{~b}}$ Università di Bologna, Bologna, Italy

${ }^{71 a}$ INFN Sezione di Catania, Catania, Italy

${ }^{71 \mathrm{~b}}$ Università di Catania, Catania, Italy

${ }^{72 \mathrm{a}}$ INFN Sezione di Firenze, Firenze, Italy

${ }^{72 \mathrm{~b}}$ Università di Firenze, Firenze, Italy

${ }^{73}$ INFN Laboratori Nazionali di Frascati, Frascati, Italy

${ }^{74 a}$ INFN Sezione di Genova, Genova, Italy

${ }^{74 \mathrm{~b}}$ Università di Genova, Genova, Italy

${ }^{75 a}$ INFN Sezione di Milano-Bicocca, Milano, Italy

${ }^{75 b}$ Università di Milano-Bicocca, Milano, Italy

${ }^{76 a}$ INFN Sezione di Napoli, Napoli, Italy

${ }^{76 \mathrm{~b}}$ Università di Napoli 'Federico II', Napoli, Italy

${ }^{76 c}$ Università della Basilicata, Potenza, Italy

${ }^{76 \mathrm{~d}}$ Università G. Marconi, Roma, Italy

${ }^{77}$ INFN Sezione di Padova, Padova, Italy

${ }^{77 b}$ Università di Padova, Padova, Italy

${ }^{77 \mathrm{c}}$ Università di Trento, Trento, Italy

${ }^{78 a}$ INFN Sezione di Pavia, Pavia, Italy

${ }^{78 b}$ Università di Pavia, Pavia, Italy

${ }^{79 a}$ INFN Sezione di Perugia, Perugia, Italy

${ }^{79 b}$ Università di Perugia, Perugia, Italy

${ }^{80 a}$ INFN Sezione di Pisa, Pisa Italy

${ }^{80 \mathrm{~b}}$ Università di Pisa, Pisa Italy

${ }^{80 \mathrm{c}}$ Scuola Normale Superiore di Pisa, Pisa Italy

${ }^{80 \mathrm{~d}}$ Università di Siena, Siena, Italy

${ }^{81 a}$ INFN Sezione di Roma, Rome, Italy

${ }^{81 \mathrm{~b}}$ Sapienza Università di Roma, Rome, Italy

${ }^{82 \mathrm{a}}$ INFN Sezione di Torino, Torino, Italy

${ }^{82 \mathrm{~b}}$ Università di Torino, Torino, Italy

${ }^{82 \mathrm{c}}$ Università del Piemonte Orientale, Novara, Italy 


\author{
${ }^{83 a}$ INFN Sezione di Trieste, Trieste, Italy \\ ${ }^{83 \mathrm{~b}}$ Università di Trieste, Trieste, Italy \\ ${ }^{84}$ Kyungpook National University, Daegu, Korea \\ ${ }^{85}$ Chonnam National University, Institute for Universe and Elementary Particles, Kwangju, Korea \\ ${ }^{86}$ Hanyang University, Seoul, Korea \\ ${ }^{87}$ Korea University, Seoul, Korea \\ ${ }^{88}$ Kyung Hee University, Department of Physics, Seoul, Republic of Korea \\ ${ }^{89}$ Sejong University, Seoul, Korea \\ ${ }^{90}$ Seoul National University, Seoul, Korea \\ ${ }^{91}$ University of Seoul, Seoul, Korea \\ ${ }^{92}$ Yonsei University, Department of Physics, Seoul, Korea \\ ${ }^{93}$ Sungkyunkwan University, Suwon, Korea \\ ${ }^{94}$ College of Engineering and Technology, American University of the Middle East (AUM), Kuwait \\ ${ }^{95}$ Riga Technical University, Riga, Latvia \\ ${ }^{96}$ Vilnius University, Vilnius, Lithuania \\ ${ }^{97}$ National Centre for Particle Physics, Universiti Malaya, Kuala Lumpur, Malaysia \\ ${ }^{98}$ Universidad de Sonora (UNISON), Hermosillo, Mexico \\ ${ }^{99}$ Centro de Investigacion y de Estudios Avanzados del IPN, Mexico City, Mexico \\ ${ }^{100}$ Universidad Iberoamericana, Mexico City, Mexico \\ ${ }^{101}$ Benemerita Universidad Autonoma de Puebla, Puebla, Mexico \\ ${ }^{102}$ Universidad Autónoma de San Luis Potosí, San Luis Potosí, Mexico \\ ${ }^{103}$ University of Montenegro, Podgorica, Montenegro \\ ${ }^{104}$ University of Auckland, Auckland, New Zealand \\ ${ }^{105}$ University of Canterbury, Christchurch, New Zealand \\ ${ }^{106}$ National Centre for Physics, Quaid-I-Azam University, Islamabad, Pakistan \\ ${ }^{107}$ AGH University of Science and Technology Faculty of Computer Science, \\ Electronics and Telecommunications, Krakow, Poland \\ ${ }^{108}$ National Centre for Nuclear Research, Swierk, Poland \\ ${ }^{109}$ Institute of Experimental Physics, Faculty of Physics, University of Warsaw, Warsaw, Poland \\ ${ }^{110}$ Laboratório de Instrumentação e Física Experimental de Partículas, Lisboa, Portugal \\ ${ }^{111}$ Joint Institute for Nuclear Research, Dubna, Russia \\ ${ }^{112}$ Petersburg Nuclear Physics Institute, Gatchina (St. Petersburg), Russia \\ ${ }^{113}$ Institute for Nuclear Research, Moscow, Russia \\ ${ }^{114}$ Institute for Theoretical and Experimental Physics named by A.I. Alikhanov of NRC \\ 'Kurchatov Institute', Moscow, Russia \\ ${ }^{115}$ Moscow Institute of Physics and Technology, Moscow, Russia \\ ${ }^{116}$ National Research Nuclear University 'Moscow Engineering Physics Institute' (MEPhI), \\ Moscow, Russia \\ ${ }^{117}$ P.N. Lebedev Physical Institute, Moscow, Russia \\ ${ }^{118}$ Skobeltsyn Institute of Nuclear Physics, Lomonosov Moscow State University, Moscow, Russia \\ ${ }^{119}$ Novosibirsk State University (NSU), Novosibirsk, Russia \\ ${ }^{120}$ Institute for High Energy Physics of National Research Centre 'Kurchatov Institute', Protvino, Russia \\ ${ }^{121}$ National Research Tomsk Polytechnic University, Tomsk, Russia \\ ${ }^{122}$ Tomsk State University, Tomsk, Russia \\ ${ }^{123}$ University of Belgrade: Faculty of Physics and VINCA Institute of Nuclear Sciences, Belgrade, Serbia \\ ${ }^{124}$ Centro de Investigaciones Energéticas Medioambientales y Tecnológicas (CIEMAT), Madrid, Spain \\ ${ }^{125}$ Universidad Autónoma de Madrid, Madrid, Spain \\ ${ }^{126}$ Universidad de Oviedo, Instituto Universitario de Ciencias y Tecnologías Espaciales de Asturias \\ (ICTEA), Oviedo, Spain \\ ${ }^{127}$ Instituto de Física de Cantabria (IFCA), CSIC-Universidad de Cantabria, Santander, Spain \\ ${ }^{128}$ University of Colombo, Colombo, Sri Lanka \\ ${ }^{129}$ University of Ruhuna, Department of Physics, Matara, Sri Lanka \\ ${ }^{130}$ CERN, European Organization for Nuclear Research, Geneva, Switzerland \\ ${ }^{131}$ Paul Scherrer Institut, Villigen, Switzerland \\ ${ }^{132}$ ETH Zurich-Institute for Particle Physics and Astrophysics (IPA), Zurich, Switzerland \\ ${ }^{133}$ Universität Zürich, Zurich, Switzerland \\ ${ }^{134}$ National Central University, Chung-Li, Taiwan \\ ${ }^{135}$ National Taiwan University (NTU), Taipei, Taiwan \\ ${ }^{136}$ Chulalongkorn University, Faculty of Science, Department of Physics, Bangkok, Thailand \\ ${ }^{137}$ Çukurova University, Physics Department, Science and Art Faculty, Adana, Turkey
}




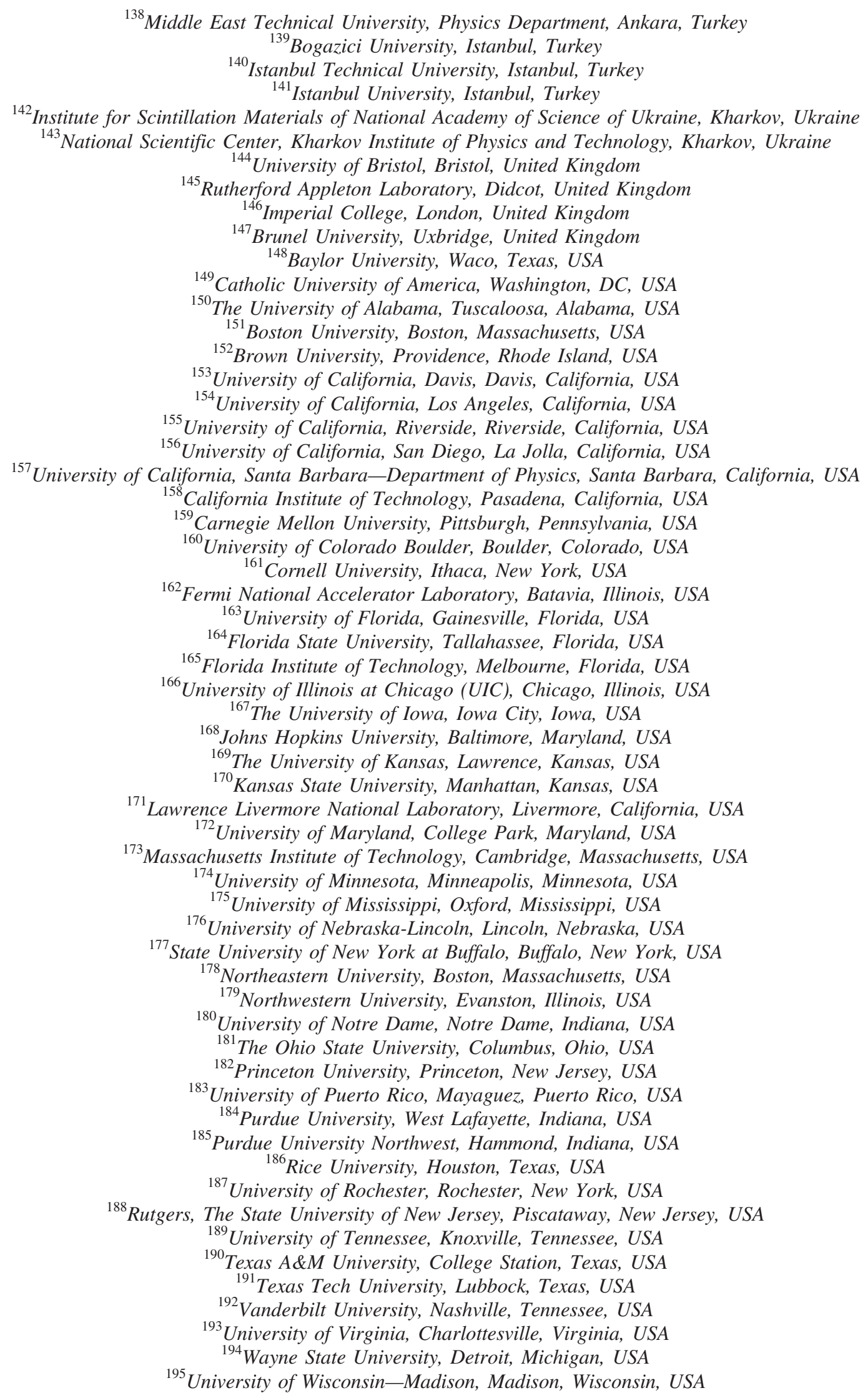

${ }^{\mathrm{a}}$ Deceased. 
${ }^{\mathrm{b}}$ Also at Vienna University of Technology, Vienna, Austria

${ }^{\mathrm{c}}$ Also at Institute of Basic and Applied Sciences, Faculty of Engineering, Arab Academy for Science, Technology and Maritime Transport, Alexandria, Egypt

${ }^{\mathrm{d}}$ Also at Université Libre de Bruxelles, Bruxelles, Belgium

${ }^{\mathrm{e}}$ Also at IRFU, CEA, Université Paris-Saclay, Gif-sur-Yvette, France

${ }^{\mathrm{f}}$ Also at Universidade Estadual de Campinas, Campinas, Brazil

${ }^{\mathrm{g}}$ Also at Federal University of Rio Grande do Sul, Porto Alegre, Brazil

${ }^{\mathrm{h}}$ Also at Universidade Federal de Mato Grosso do Sul, Nova Andradina, Brazil

${ }^{\mathrm{i}}$ Also at Universidade Federal de Pelotas, Pelotas, Brazil

${ }^{\mathrm{j}}$ Also at University of Chinese Academy of Sciences, Bejing, China

${ }^{k}$ Also at Institute for Theoretical and Experimental Physics named by A.I. Alikhanov of NRC 'Kurchatov Institute', Moscow, Russia

${ }^{1}$ Also at Joint Institute for Nuclear Research, Dubna, Russia

${ }^{\mathrm{m}}$ Also at Ain Shams University, Cairo, Egypt

${ }^{\mathrm{n}}$ Also at British University in Egypt, Cairo, Egypt

${ }^{\circ}$ Also at Cairo University, Cairo, Egypt

${ }^{\mathrm{p}}$ Also at Fayoum University, El-Fayoum, Egypt

${ }^{\mathrm{q}}$ Also at Purdue University, West Lafayette, Indiana, USA

${ }^{\mathrm{r}}$ Also at Université de Haute Alsace, Mulhouse, France

${ }^{\mathrm{s}}$ Also at Ilia State University, Tbilisi, Georgia

${ }^{t}$ Also at Erzincan Binali Yildirim University, Erzincan, Turkey

"Also at CERN, European Organization for Nuclear Research, Geneva, Switzerland

${ }^{v}$ Also at RWTH Aachen University, III. Physikalisches Institut A, Aachen, Germany

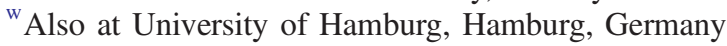

${ }^{x}$ Also at Department of Physics, Isfahan University of Technology, Isfahan, Iran

${ }^{y}$ Also at Brandenburg University of Technology, Cottbus, Germany

${ }^{z}$ Also at Skobeltsyn Institute of Nuclear Physics, Lomonosov Moscow State University, Moscow, Russia

${ }^{\text {aa }}$ Also at Institute of Physics, University of Debrecen, Debrecen, Hungary

${ }^{\mathrm{bb}}$ Also at Physics Department, Faculty of Science, Assiut University

${ }^{c c}$ Also at MTA-ELTE Lendület CMS Particle and Nuclear Physics Group, Eötvös Loránd University, Budapest, Hungary

${ }^{\mathrm{dd}}$ Also at Institute of Nuclear Research ATOMKI, Debrecen, Hungary

${ }^{\text {ee }}$ Also at IIT Bhubaneswar, Bhubaneswar, India

${ }^{\mathrm{ff}}$ Also at Institute of Physics, Bhubaneswar, India

${ }^{g g}$ Also at G.H.G. Khalsa College, Punjab, India

${ }^{\text {hh }}$ Also at Shoolini University, Solan, India

${ }^{\text {ii }}$ Also at University of Hyderabad, Hyderabad, India

${ }^{\mathrm{jj}}$ Also at University of Visva-Bharati, Santiniketan, India

${ }^{\mathrm{kk}}$ Also at Indian Institute of Technology (IIT), Mumbai, India

"Also at Deutsches Elektronen-Synchrotron, Hamburg, Germany

${ }^{\mathrm{mm}}$ Also at Department of Physics, University of Science and Technology of Mazandaran, Behshahr, Iran

${ }^{\text {nn }}$ Also at INFN Sezione di Bari, Università di Bari, Politecnico di Bari, Bari, Italy

${ }^{\circ 0}$ Also at Italian National Agency for New Technologies, Energy and Sustainable Economic Development

${ }^{\mathrm{pp}}$ Also at Centro Siciliano di Fisica Nucleare e di Struttura Della Materia

${ }^{\mathrm{qq}}$ Also at Riga Technical University, Riga, Latvia

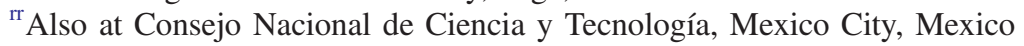

${ }^{\text {ss }}$ Also at Warsaw University of Technology, Institute of Electronic Systems, Warsaw, Poland

${ }^{\text {th }}$ Also at Institute for Nuclear Research, Moscow, Russia

${ }^{\text {u }}$ Also at National Research Nuclear University 'Moscow Engineering Physics Institute' (MEPhI), Moscow, Russia

${ }^{\mathrm{vv}}$ Also at Institute of Nuclear Physics of the Uzbekistan Academy of Sciences, Tashkent, Uzbekistan

${ }^{w w}$ Also at St. Petersburg State Polytechnical University, St. Petersburg, Russia

${ }^{x x}$ Also at University of Florida, Gainesville, Florida, USA

${ }^{\text {yy }}$ Also at Imperial College, London, United Kingdom

${ }^{\mathrm{zz}}$ Also at P.N. Lebedev Physical Institute, Moscow, Russia

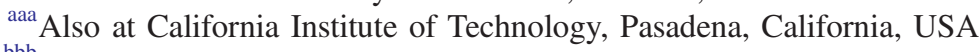

${ }^{b b b}$ Also at Budker Institute of Nuclear Physics, Novosibirsk, Russia

${ }^{c c c}$ Also at Faculty of Physics, University of Belgrade, Belgrade, Serbia

ddd Also at Trincomalee Campus, Eastern University, Sri Lanka

${ }^{e e e}$ Also at INFN Sezione di Pavia, Università di Pavia, Pavia, Italy

${ }^{\text {fff }}$ Also at National and Kapodistrian University of Athens, Athens, Greece

${ }^{\mathrm{ggg}} \mathrm{Also}$ at Universität Zürich, Zurich, Switzerland 
${ }^{\text {hhh }}$ Also at Stefan Meyer Institute for Subatomic Physics, Vienna, Austria

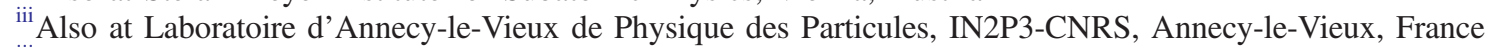

${ }^{\mathrm{jjj}}$ Also at Şırnak University, Şırnak, Turkey

${ }^{k k k}$ Also at Department of Physics, Tsinghua University, Beijing, China

${ }^{111}$ Also at Near East University, Research Center of Experimental Health Science, Nicosia, Turkey

${ }^{\mathrm{mmm}}$ Also at Beykent University, Istanbul, Turkey

${ }^{\mathrm{nnn}}$ Also at Istanbul Aydin University, Application and Research Center for Advanced Studies (App. \& Res. Cent. for Advanced Studies)

${ }^{\mathrm{ooo}}$ Also at Mersin University, Mersin, Turkey

${ }^{\mathrm{ppp}}$ Also at Piri Reis University, Istanbul, Turkey

${ }^{\mathrm{qqq}}$ Also at Adiyaman University, Adiyaman, Turkey

${ }^{\mathrm{rrr}}$ Also at Ozyegin University, Istanbul, Turkey

${ }^{\text {sss }}$ Also at Izmir Institute of Technology, Izmir, Turkey

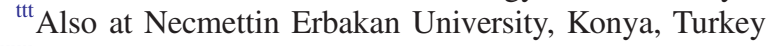

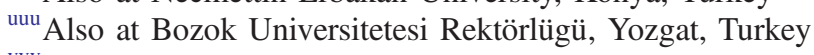

${ }^{\mathrm{vvv}}$ Also at Marmara University, Istanbul, Turkey

${ }^{\text {www }}$ Also at Milli Savunma University

${ }^{\mathrm{xxx}}$ Also at Kafkas University, Kars, Turkey

${ }^{\text {yyy }}$ Also at Istanbul Bilgi University, Istanbul, Turkey

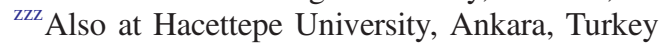

${ }^{\text {aaaa }}$ Also at School of Physics and Astronomy, University of Southampton, Southampton, United Kingdom

${ }^{b b b b}$ Also at IPPP Durham University, Durham, England

${ }^{\mathrm{cccc}}$ Also at Monash University, Faculty of Science, Clayton, Australia

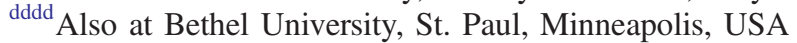

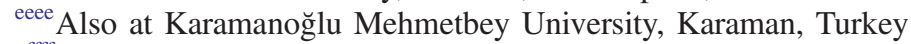

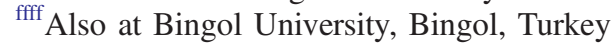

${ }^{\text {gggg }}$ Also at Georgian Technical University, Tbilisi, Georgia

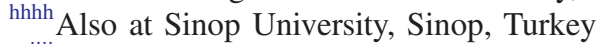

iiii Also at Mimar Sinan University, Istanbul, Istanbul, Turkey

${ }^{j j j j}$ Also at Nanjing Normal University Department of Physics

${ }^{\text {kkkk }}$ Also at Texas A\&M University at Qatar, Doha, Qatar

${ }^{1111}$ Also at Kyungpook National University, Daegu, Korea 